\title{
AUTOMORPHY FOR SOME $l$-ADIC LIFTS OF AUTOMORPHIC MOD $l$ GALOIS REPRESENTATIONS. II
}

\author{
by RiCHARD TAYLOR*
}

\begin{abstract}
We extend the results of [CHT] by removing the 'minimal ramification' condition on the lifts. That is we establish the automorphy of suitable conjugate self-dual, regular (de Rham with distinct Hodge-Tate numbers), $l$-adic lifts of certain automorphic mod $l$ Galois representations of any dimension. The main innovation is a new approach to the automorphy of non-minimal lifts which is closer in spirit to the methods of [TW] than to those of [W], which relied on Ihara's lemma.
\end{abstract}

\section{Introduction}

This paper is a sequel to [CHT]. Both papers prove modularity lifting theorems for representations of any dimension. In [CHT] we proved that certain minimally ramified $l$-adic lifts of automorphic mod $l$ representations are themselves automorphic. We also showed how a conjecture (which we called 'Ihara's lemma') about the space of mod $l$ automorphic forms on definite unitary groups would imply that one could extend this results to lifts which are not minimally ramified. In this paper we prove such a result unconditionally by means of a different approach. Instead of trying to generalise Wiles' level raising arguments [W], as we did in [CHT], we have found a development of the arguments used in the minimal case (see [TW]), which also applies in the non-minimal setting. Ideas of Kisin (see $[\mathrm{K}]$ ) were very influential in the development of our arguments. The results of this paper imply that all the theorems of [HSBT] also become unconditional. For instance we prove the Sato-Tate conjecture for an elliptic curve over a totally real field with somewhere multiplicative reduction.

The following is an example of the main modularity lifting theorems proved in this paper. We emphasise that the proof of this theorem depends on the machinery developed in [CHT].

Theorem A. - Let $n \in \mathbf{Z}_{\geq 1}$ be even and let $l>\max \{3, n\}$ be a prime. Let

$$
r: \operatorname{Gal}(\overline{\mathbf{Q}} / \mathbf{Q}) \longrightarrow \operatorname{GSp}_{n}\left(\mathbf{Z}_{l}\right)
$$

be a continuous irreducible representation with the following properties.

\footnotetext{
^ Partially supported by NSF Grant DMS-0100090.
} 
1. $r$ ramifies at only finitely many primes.

2. $\left.r\right|_{\mathrm{Gal}\left(\overline{\mathbf{Q}}_{l} / \mathbf{Q}_{l}\right)}$ is crystalline and $\operatorname{dim}_{\mathbf{Q}_{l}} \operatorname{gr}^{i}\left(r \otimes_{\mathbf{Q}_{l}} \mathrm{~B}_{\mathrm{DR}}\right)^{\mathrm{Gal}\left(\overline{\mathbf{Q}}_{l} / \mathbf{Q}_{l}\right)}=0$ unless $i \in\{0,1$, $\ldots, n-1\}$, in which case it has dimension 1 .

3. There is a prime $q \neq l$ such that the semisimplification $\left.r\right|_{\mathrm{G}_{\mathbf{Q}_{q}}} ^{\mathrm{s}}$ is unramified and $r{ }_{\mathrm{G}_{\mathbf{Q}_{q}}}^{\mathrm{ss}}\left(\mathrm{Frob}_{q}\right)$ has eigenvalues $\left\{\alpha q^{i}: i=0,1, \ldots, n-1\right\}$ for some $\alpha$.

4. The image of $r \bmod l$ contains $\operatorname{Sp}_{n}\left(\mathbf{F}_{l}\right)$.

5. $r \bmod l$ arises from a cuspidal automorphic representation $\pi_{0}$ of $\mathrm{GL}_{n}(\mathbf{A})$ for which $\pi_{0, \infty}$ has trivial infinitesimal character and $\pi_{0, q}$ is an unramified twist of the Steinberg representation.

Then $r$ arises from a cuspidal automorphic representation $\pi$ of $\mathrm{GL}_{n}(\mathbf{A})$ for which $\pi_{\infty}$ has trivial infinitesimal character and $\pi_{q}$ is an unramified twist of the Steinberg representation.

Let us comment on the conditions in this theorem. The fourth condition is used to make the Cebotarev argument in the Taylor-Wiles method work. Much weaker conditions are possible. (See Theorem 5.4.) One expects to need to assume that $r$ is de Rham at $l$. The stronger assumption that it be crystalline and that the Hodge-Tate numbers lie in a range which is small compared to $l$ is imposed so that one can use the theory of Fontaine and Laffaille to calculate the relevant local deformation ring at $l$. The assumptions that $r$ is valued in the symplectic (or similar) group and that the Hodge-Tate numbers are different are needed so that the numerology behind the Taylor-Wiles method works out. This is probably essential to the method. The condition on $\left.r\right|_{\mathrm{G}_{\mathbf{Q}_{q}}}$ for some prime $q$ says that the representation looks as if it could correspond under the local Langlands correspondence to a Steinberg representation. We need the existence of such a prime $q$ so that we can transfer the relevant automorphic forms to and from unitary groups and so that we can attach Galois representations to them. As the trace formula technology improves one may be able to relax this condition.

As applications the following results proved conditionally in [HSBT] now become unconditional.

Theorem B. - Let $\mathrm{K}$ be a totally real field and $\mathrm{E} / \mathrm{K}$ be an elliptic curve with multiplicative reduction at some prime.

1. For any odd integer $m$ there is a finite Galois totally real extension $\mathrm{L} / \mathrm{K}$ such that Symm $^{m} \mathrm{H}^{1}(\mathrm{E})$ becomes automorphic over $\mathrm{L}$. (One can choose an $\mathrm{L}$ that will work simultaneously for any finite set of odd positive integers.)

2. For any positive integer $m$ the $\mathrm{L}$-function $\mathrm{L}\left(\mathrm{Symm}^{m} \mathrm{H}^{1}(\mathrm{E}), s\right)$ has meromorphic continuation to the whole complex plane and satisfies the expected functional equation. It is holomorphic and non-zero in the region $\operatorname{Re} s \geq 1+m / 2$. 
3. The Sato-Tate conjecture is true for $\mathrm{E}$, i.e. the numbers

$$
\left(1+p-\# \mathrm{E}\left(\mathbf{F}_{p}\right)\right) / 2 \sqrt{p}
$$

are equidistributed in $[-1,1]$ with respect to the measure $(2 / \pi) \sqrt{1-t^{2}} d t$.

The condition that $\mathrm{E}$ has multiplicative reduction at some prime results from condition 3 of Theorem A (and its generalisations). We hope that this condition could be removed as trace formula technology improves.

Theorem C. - Suppose that $n$ is an even, positive integer, and that $t \in \mathbf{Q}-$ $\mathbf{Z}[1 /(n+1)]$. Let $\mathbf{Y}_{t}$ denote the projective variety

$$
\left(\mathrm{X}_{0}^{n+1}+\mathrm{X}_{1}^{n+1}+\ldots+\mathrm{X}_{n}^{n+1}\right)=(n+1) t \mathrm{X}_{0} \mathrm{X}_{1} \ldots \mathrm{X}_{n}
$$

It carries an action of

$$
\mathrm{H}^{\prime}=\operatorname{ker}\left(\boldsymbol{\mu}_{n+1}^{n+1} \stackrel{\prod}{\longrightarrow} \boldsymbol{\mu}_{n+1}\right)
$$

acting by multiplication on the coordinates. Then the $\mathrm{L}$-function $\mathrm{L}\left(\mathrm{V}_{t}, s\right)$ of

$$
\mathrm{H}^{n-1}\left(\mathrm{Y}_{(1: t)} \times \overline{\mathbf{Q}}, \mathbf{Q}_{l}\right)^{\mathrm{H}^{\prime}}
$$

is independent of $l$, has meromorphic continuation to the whole complex plane and satisfies the expected functional equation

$$
\mathrm{L}\left(\mathrm{V}_{t}, s\right)=\epsilon\left(\mathrm{V}_{t}, s\right) \mathrm{L}\left(\mathrm{V}_{t}, n-s\right) .
$$

(See Theorem 6.5 for details. A referee asked us to explain the importance of this particular family. It is a family that has been studied quite widely and has some beautiful properties, but for us the main interest of this family is that $\operatorname{Gal}(\overline{\mathbf{Q}} / \mathbf{Q})$ typically has image $\operatorname{GSp}_{n}\left(\mathbf{Z}_{l}\right)$ in $\operatorname{Aut}^{n-1}\left(\mathrm{Y}_{(1: t)} \times \overline{\mathbf{Q}}, \mathbf{Q}_{l}\right)^{\mathrm{H}^{\prime}}$. Note that $\operatorname{dim}_{\mathbf{Q}_{l}} \mathrm{H}^{n-1}\left(\mathrm{Y}_{(1: t)} \times \overline{\mathbf{Q}}, \mathbf{Q}_{l}\right)^{\mathrm{H}^{\prime}}=n$. Thus this theorem is illustrative that our techniques allow one to study the L-function of varieties for which the image of Galois acting on the $l$-adic cohomology is large.)

Next let us outline our argument for Theorem A in the case of one nonminimal prime $p$. We will assume that $p \equiv 1 \bmod l$ and that the representation has unipotent ramification at $p$. One can use base change arguments to reduce to this case. We consider simultaneously two deformation problems. Let $\sigma$ denote a generator of the tame inertia in $\operatorname{Gal}\left(\overline{\mathbf{Q}}_{p} / \mathbf{Q}_{p}\right)$. In the first deformation problem we consider lifts in which $\sigma$ has characteristic polynomial $(\mathrm{X}-1)^{n}$. In the second we consider those in which $\sigma$ has characteristic polynomial $\left(\mathrm{X}-\zeta_{1}\right) \ldots\left(\mathrm{X}-\zeta_{n}\right)$ where the $\zeta_{i}$ are distinct $l^{\text {th }}$ roots of 1 . We need to use Kisin's framed deformations. Let $\mathrm{R}_{p}^{(1)}$ 
and $\mathrm{R}_{p}^{(2)}$ denote the two local framed deformation spaces. Then $\mathrm{R}_{p}^{(1)} / \lambda=\mathrm{R}_{p}^{(2)} / \lambda$ (where $\lambda$ is the prime above $l$ in the coefficient ring) and these rings have dimension $n^{2}+1$. Moreover $\mathbf{R}_{p}^{(2)}$ is irreducible, whereas $\mathbf{R}_{p}^{(1)}$ is reducible, but the irreducible components of $\mathrm{R}_{p}^{(1)}$ and $\mathrm{R}_{p}^{(1)} / \lambda$ are in bijection under the reduction map.

We use a simple variant of the usual Taylor-Wiles patching argument to create two limiting framed, global deformation rings $\mathrm{R}^{(1)}=\mathrm{R}_{p}^{(1)}\left[\left[\mathrm{Y}_{1}, \ldots, \mathrm{Y}_{r}\right]\right]$ and $\mathrm{R}^{(2)}=\mathrm{R}_{p}^{(2)}\left[\left[\mathrm{Y}_{1}, \ldots, \mathrm{Y}_{r}\right]\right]$ together with two limiting, framed spaces of automorphic forms $\mathrm{H}^{(1)}$ and $\mathrm{H}^{(2)}$. As usual, the method yields that $\mathrm{H}^{(i)}$ has $\mathrm{R}^{(i)}$-depth $\geq$ $n^{2}+1+r$. (One uses the diamond operators to see this.) Moreover we can compatibly identify $\mathrm{R}^{(1)} / \lambda \cong \mathrm{R}^{(2)} / \lambda$ and $\mathrm{H}^{(1)} / \lambda \cong \mathrm{H}^{(2)} / \lambda$. It suffices to prove that all primes of $\mathrm{R}^{(1)}$ are in the support of $\mathrm{H}^{(1)}$.

As $\mathrm{R}^{(2)}$ is irreducible of dimension $n^{2}+1+r$ and $\mathrm{H}^{(2)}$ has $\mathrm{R}^{(2)}$-depth $\geq$ $n^{2}+1+r$, as usual we conclude that every prime of $\mathrm{R}^{(2)}$ is in the support of $\mathrm{H}^{(2)}$. Hence every prime of $\mathrm{R}^{(2)} / \lambda \cong \mathrm{R}^{(1)} / \lambda$ is in the support of $\mathrm{H}^{(2)} / \lambda \cong \mathrm{H}^{(1)} / \lambda$. Because the irreducible components of $\mathrm{R}^{(1)}$ and $\mathrm{R}^{(1)} / \lambda$ are in bijection under reduction (because in turn the same is true for the local ring $\mathrm{R}_{p}^{(1)}$ by explicit computation) we can conclude that all primes of $\mathrm{R}^{(1)}$ are in the support of $\mathrm{H}^{(1)}$, as desired.

The main point is that Kisin's variant of the Taylor-Wiles method works well for the second deformation problem. One would like to use this and the fact that the first and second problems become equal modulo $\lambda$, to deduce an $\mathrm{R}=\mathrm{T}$ theorem for the first problem also. This does not seem to be possible at finite level. It is however possible at 'infinite level' because the 'deformation ring' $\mathrm{R}^{(1)}$ has such a simple form. (Specifically it has the property that its irreducible components are in bijection with the irreducible components of its reduction modulo $\lambda$.)

The reader of this paper will need to make frequent reference to the first three chapters of [CHT]. However we will make no appeal to the fourth chapter of [CHT], nor to the appendices to that paper.

Section 2 of this paper contains some algebraic background: some simple lemmas in commutative algebra and a discussion of the moduli space of nilpotent matrices. These results may well be well known, but for the lack of a suitable reference we include proofs here. The sole purpose of the second section is to prove Proposition 3.1, which describes the structure of certain universal lifting rings for representations of local Galois groups. Because the structure of the maximal tame quotient of the local Galois group is so simple, the arguments in this section are mostly about moduli spaces of pairs of matrices. In earlier versions of this preprint these arguments were unfortunately incomplete. Section 4 starts by reviewing notation and background from [CHT] and then gives the key argument of this paper to prove the equality of a Hecke algebra and of the quotient of a universal deformation ring for a representation of a global Galois group by its nilradical (see 
Theorem 4.1). In Section 5 this theorem is combined with the theory of base change to prove more user friendly modularity lifting theorems. The arguments of Section 5 are taken directly from [CHT]. Indeed parts of Sections 4 and 5 are repeated almost verbatim from [CHT]. We hope that this will make the present paper easier to read. Finally Section 6 lists several consequences of our main modularity lifting theorems.

It is a pleasure to acknowledge the debt this work owes to the ideas of Skinner and Wiles [SW] and particularly of Kisin [K]. I am also grateful to Brian Conrad and Dennis Gaitsgory for useful conversations concerning Section 2. It is a pleasure to thank my collaborators on earlier stages of this project: Laurent Clozel, Michael Harris and Nick Shepherd-Barron. It will be clear to the reader how much the main theorems of this paper owe to our earlier joint work [CHT] and [HSBT]. Finally I would like to thank the referees for their careful and very helpful work. I believe their suggestions have significantly improved the readability of this paper (and of [CHT]).

\section{Some commutative algebra}

In this section we will review some results from commutative algebra that we will need. It is possible that these are all clear to experts, but, not myself being an expert, I have chosen to present them in some detail. We start with a definition and two lemmas that will play an important role in our modularity argument.

We will let $l$ denote a prime number and $\mathrm{K}$ a finite extension of $\mathbf{Q}_{l}$ with ring of integers $\mathscr{O}$, maximal ideal $\lambda$ and residue field $k=\mathscr{O} / \lambda$.

Definition 2.1. - Let $\mathrm{A}$ denote a noetherian local ring. If $\mathrm{M}$ is a finitely generated $\mathrm{A}$ module the following are equivalent.

$-\operatorname{Ann}_{\mathrm{A}}(\mathrm{M})$ is nilpotent.

- All minimal primes of $\mathrm{A}$ are in the support of $\mathrm{M}$.

- All primes of $\mathrm{A}$ are in the support of $\mathrm{M}$.

If these conditions are satisfied we call $\mathrm{M}$ a nearly faithful A-module.

If $\mathrm{A}$ is reduced then faithful and nearly faithful are synonymous.

Lemma 2.2. - Suppose $\mathrm{A}$ is a noetherian local ring and that $\mathrm{M}$ is a finitely generated A-module.

1. If $\mathrm{I}$ is an ideal of $\mathrm{A}$ and $\mathrm{M}$ is a nearly faithful $\mathrm{A}$-module then $\mathrm{M} / \mathrm{IM}$ is a nearly faithful A/I-module. In particular, if $\mathrm{J} \supset \mathrm{I}$ is another ideal and the action of $\mathrm{A}$ on $\mathrm{M} / \mathrm{IM}$ factors through $\mathrm{A} / \mathrm{J}$ then $\mathrm{J} \subset \sqrt{ } \mathrm{I}$ and $\mathrm{M} / \mathrm{IM}$ is a nearly faithful $\mathrm{A} / \mathrm{J}-$ module. 
2. Suppose that $\mathrm{A}$ is an excellent (or just catenary) local $\mathscr{O}$-algebra, that each irreducible component of SpecA has the same dimension and that all the generic points of Spec A have characteristic zero. Suppose also that every prime of A minimal over $\lambda \mathrm{A}$ contains a unique minimal prime of $\mathrm{A}$. Suppose finally that $\mathrm{M}$ is an $\mathrm{A}$-module which is $\mathscr{O}$-torsion free. If $\mathrm{M} / \lambda \mathrm{M}$ is a nearly faithful $\mathrm{A} / \lambda \mathrm{A}$-module, then $\mathrm{M}$ is a nearly faithful A-module.

Proof. - Consider the first part. If $\wp \supset \mathrm{I}$ is a prime ideal we need to show that $(\mathrm{M} / \mathrm{IM})_{\wp} \neq(0)$. If not then

$$
\mathrm{M}_{\wp} / \mathrm{I}_{\wp} \mathrm{M}_{\wp}=(\mathrm{M} / \mathrm{IM})_{\wp}=(0)
$$

and so by Nakayama's lemma $\mathrm{M}_{\wp}=(0)$, a contradiction to $\wp$ being in the support of $\mathrm{M}$.

Now consider the second part. Let $\mathrm{P}$ be a minimal prime of $\mathrm{A}$. Let $\wp$ be a minimal prime containing $(\lambda, \mathrm{P})$. As $\mathrm{A}$ is catenary (because it is excellent) the Krull dimension of $\mathrm{A} / \wp$ is one less that the Krull dimension of $\mathrm{A} / \mathrm{P}$. If $\mathfrak{q}$ is any prime properly contained in $\wp$ we see that $\mathrm{A} / \mathfrak{q}$ has Krull dimension at least the Krull dimension of $\mathrm{A} / \mathrm{P}$. Thus $\mathfrak{q}$ is minimal and so does not contain $\lambda \mathrm{A}$. Thus $\wp$ is a minimal prime containing $\lambda \mathrm{A}$, and so by our assumption the only prime of $\mathrm{A}$ properly contained in $\wp$ is $\mathrm{P}$, i.e. $\mathrm{A}_{\wp}$ has only two primes $\wp_{\wp}$ and $\mathrm{P}_{\wp}$. As

$$
\mathrm{M}_{\wp} / \lambda \mathrm{M}_{\wp}=(\mathrm{M} / \lambda \mathrm{M})_{\wp} \neq(0)
$$

we deduce that $\mathrm{M}_{\wp} \neq(0)$ and that $\mathrm{Ann}_{\mathrm{A}_{\wp}} \mathrm{M}_{\wp} \subset \wp_{\wp}$. Thus $\sqrt{ }\left(\mathrm{Ann}_{\mathrm{A}_{\wp}} \mathrm{M}_{\wp}\right)=\wp_{\wp}$ or $\mathrm{P}_{\wp}$.

Let $\varpi$ denote a generator of $\lambda$. For all $r \in \mathbf{Z}_{>0}$ the map $\varpi^{r}: \mathbf{M} \hookrightarrow \mathbf{M}$ is injective and hence $\varpi^{r}: \mathbf{M}_{\wp} \hookrightarrow \mathbf{M}_{\wp}$. Thus $\varpi^{r} \notin \operatorname{Ann}_{\mathrm{A}_{\wp}} \mathbf{M}_{\wp}$ and so $\varpi \notin$ $\sqrt{ }\left(\mathrm{Ann}_{\mathrm{A}_{\wp}} \mathrm{M}_{\wp}\right)$. We conclude that $\sqrt{ }\left(\mathrm{Ann}_{\mathrm{A}_{\wp}} \mathrm{M}_{\wp}\right)=\mathrm{P}_{\wp}$, so that $\mathrm{P}_{\wp} \supset \mathrm{Ann}_{\mathrm{A}_{\wp}} \mathrm{M}_{\wp}$. Thus

$$
\mathrm{M}_{\mathrm{P}}=\left(\mathrm{M}_{\wp}\right)_{\mathrm{P}_{\wp}} \neq(0)
$$

Lemma 2.3. - Suppose that $\mathrm{A}$ is a noetherian local ring and that $\mathrm{M}$ is a finitely generated A-module. Suppose that the $\mathfrak{m}_{\mathrm{A}}$-depth of $\mathrm{M}$ is greater than or equal to the Krull dimension of $\mathrm{A}$. Then the $\mathrm{m}_{\mathrm{A}}$-depth of $\mathrm{M}$ equals the Krull dimension of $\mathrm{A}$, and the support of $\mathrm{M}$ is equal to a union of irreducible components of Spec A. In particular if Spec $\mathrm{A}$ is irreducible then $\mathrm{M}$ is a nearly faithful A-module.

Proof. - By Proposition 18.2 of [E] every associated prime of $\mathrm{M}$ has dimension at least the $\mathfrak{m}_{\mathrm{A}}$-depth of $\mathrm{M}$. Thus every minimal prime over $\mathrm{Ann}_{\mathrm{A}} \mathrm{M}$ (which will then be an associated prime of $\mathrm{M}$ by Theorem 3.1a of [E]) has dimension at least the Krull dimension of $\mathrm{A}$, and so must be a minimal prime of $\mathrm{A}$. 
The rest of this section is only required for the proof of Proposition 3.1. First we will consider some results about polynomials and matrices. Let $\operatorname{Pol}_{n} / \mathscr{O}$ denote the space of monic polynomials of degree $n$ (i.e. simply affine $n$-space over $\mathscr{O}$ ). There is a finite flat map

$$
\begin{gathered}
\text { Aff }^{n} \longrightarrow \operatorname{Pol}_{n} \\
\left(\alpha_{1}, \ldots, \alpha_{n}\right) \longmapsto \prod_{i=1}^{n}\left(\mathrm{X}-\alpha_{i}\right) .
\end{gathered}
$$

If $f(t) \in \mathscr{O}[t]$ there is a unique morphism

$$
\begin{aligned}
\mathrm{Pol}_{n} & \longrightarrow \mathrm{Pol}_{n} \\
\mathrm{P} & \longmapsto \mathrm{P}_{f}
\end{aligned}
$$

such that

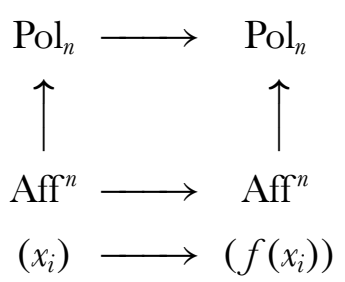

commutes, i.e. such that

$$
\left(\prod_{i=1}^{n}\left(\mathrm{X}-\alpha_{i}\right)\right)_{f}=\prod_{i}\left(\mathrm{X}-f\left(\alpha_{i}\right)\right) .
$$

Note that if $\mathrm{R}$ is a $\mathscr{O}$-algebra and $\mathrm{A}$ is an $n \times n$ matrix over $\mathrm{R}$ then

$$
\operatorname{char}_{f(\mathrm{~A})}(\mathrm{X})=\operatorname{char}_{\mathrm{A}}(\mathrm{X})_{f},
$$

where $\operatorname{char}_{\mathrm{A}}$ denotes the characteristic polynomial of A. (To see this one can reduce to the case of $\mathrm{A}=\left(\mathrm{X}_{i j}\right)$ over $\mathscr{O}\left[\mathrm{X}_{i j}\right]_{i, j=1, \ldots, n}$, and then work over the algebraic closure of the field of fractions of $\mathscr{O}\left[\mathrm{X}_{i j}\right]_{i, j=1, \ldots, n}$, where $\mathrm{A}$ can be diagonalised.) The following lemma follows easily from this, the Cayley-Hamilton theorem and standard power series identities.

Lemma 2.4. - Suppose that $l \geq n$ and that $\mathrm{R}$ is a $\mathscr{O}$-algebra. If $\mathrm{A} \in \mathrm{M}_{n}(\mathrm{R})$ has characteristic polynomial $\mathrm{X}^{n}$ then

$$
\exp (\mathrm{A})=1_{n}+\mathrm{A}+\mathrm{A}^{2} / 2 !+\ldots+\mathrm{A}^{n-1} /(n-1) !
$$

has characteristic polynomial $(\mathrm{X}-1)^{n}$. If $\mathrm{B} \in \mathrm{M}_{n}(\mathrm{R})$ has characteristic polynomial $(\mathrm{X}-1)^{n}$ then

$$
\log (\mathrm{B})=\left(\mathrm{B}-1_{n}\right)-\left(\mathrm{B}-1_{n}\right)^{2} / 2+\ldots+(-1)^{n}\left(\mathrm{~B}-1_{n}\right)^{n-1} /(n-1)
$$

has characteristic polynomial $\mathrm{X}^{n}$. Moreover we have the following well known identities: 


$$
\begin{aligned}
& -\exp \left(\mathrm{CAC}^{-1}\right)=\mathrm{C} \exp (\mathrm{A}) \mathrm{C}^{-1} \text { and } \log \left(\mathrm{CBC}^{-1}\right)=\mathrm{C} \log (\mathrm{B}) \mathrm{C}^{-1} ; \\
& -\log \exp \mathrm{A}=\mathrm{A} \text { and } \exp \log \mathrm{B}=\mathrm{B} ; \\
& -\exp (m \mathrm{~A})=\exp (\mathrm{A})^{m} \text { and } \log \left(\mathrm{B}^{m}\right)=m \log \mathrm{B} \text { for any positive integer } m .
\end{aligned}
$$

(This lemma remains true if $\mathrm{R}$ is any ring in which $(n-1)$ ! is invertible.)

Let $\mathscr{P}_{n}$ denote the set of partitions $n=n_{1}+\ldots+n_{r}$ where the $n_{i}$ are positive integers and their order does not matter. For any partition $\sigma=\left\{n_{1}, \ldots, n_{r}\right\}$ we set $d_{0}(\sigma)=0$ and define recursively $d_{i}(\sigma)$ to be $d_{i-1}(\sigma)$ plus the number of $j$ for which $n_{j} \geq i$. This sets up a bijection between $\mathscr{P}_{n}$ and the set of non-decreasing sequences of integers

$$
0=d_{0} \leq d_{1} \leq \ldots \leq d_{n-1} \leq d_{n}=n
$$

We define a partial order $\geq$ on $\mathscr{P}_{n}$ by setting $\sigma \geq \sigma^{\prime}$ if $d_{i}(\sigma) \geq d_{i}\left(\sigma^{\prime}\right)$ for all $i=1, \ldots, n$. We write $\sigma>\sigma^{\prime}$ if $\sigma \geq \sigma^{\prime}$ and $\sigma \neq \sigma^{\prime}$. If $\sigma, \sigma^{\prime} \in \mathscr{P}_{n}$ we will denote by $\sigma \vee \sigma^{\prime}$ the partition corresponding to $d_{0} \leq d_{1} \leq d_{2} \leq \ldots \leq d_{n}$ where $d_{i}$ denotes the maximum of $d_{i}(\sigma)$ and $d_{i}\left(\sigma^{\prime}\right)$. Thus $\sigma \vee \sigma^{\prime}$ is the unique $\geq$-smallest partition greater than or equal to both $\sigma$ and $\sigma^{\prime}$.

For $\sigma=\left\{n_{1}, \ldots, n_{r}\right\} \in \mathscr{P}_{n}$ and for $q \in \mathscr{O}$ we will define a reduced closed subscheme $\operatorname{Pol}_{n}(\sigma, q) \subset \mathrm{Pol}_{n}$ as follows. Let $\Lambda$ be the linear subspace of $\mathrm{Aff}^{n}$ defined by

$$
\mathrm{X}_{i+1}=q \mathrm{X}_{i}
$$

for all $i=1, \ldots, n-1$ except $i=n_{1}$ or $n_{1}+n_{2}$ or $\ldots$ or $n_{1}+\ldots+n_{r-1}$. Let $\operatorname{Pol}_{n}(\sigma, q)$ denote the reduced subscheme of the scheme theoretic image of $\Lambda$ in $\operatorname{Pol}_{n}$. For any field $\mathrm{L}$ the L-points of $\operatorname{Pol}_{n}(\sigma, q)$ are those monic polynomials whose multiset of roots can be partitioned into $r$ sub-multisets each of the form $\left\{\alpha, q \alpha, \ldots, q^{n_{j}-1} \alpha\right\}$. If $q^{n} \neq 1$ in $\mathrm{K}$ and if $\sigma=\{n\}$ then over

$$
\operatorname{Pol}_{n}(\sigma, q) \times \times_{\text {Spec } \mathscr{O}} \operatorname{Spec} \mathrm{K}
$$

the universal polynomial

$$
\mathrm{P}(\mathrm{X})=\mathrm{X}^{n}-a_{1} \mathrm{X}^{n-1}+\ldots+(-1)^{n} a_{n}
$$

satisfies

$$
\mathrm{P}(\mathrm{X})=\prod_{i=1}^{n}\left(\mathrm{X}-a_{1} q^{i-1} /\left(1+q+\ldots+q^{n-1}\right)\right) .
$$

If $m$ is a positive integer and if $q \in \mathscr{O}^{\times}$we will write $\mathrm{N}(m)$ for the $m \times m$ matrix with $\mathrm{N}(m)_{i j}=1$ if $j=i+1$ and $=0$ otherwise; and $\Phi(m, q)$ for the 
$m \times m$ diagonal matrix with entries $\left(q^{m-1}, \ldots, q, 1\right)$. If $\sigma=\left(n_{1}, \ldots, n_{r}\right) \in \mathscr{P}_{n}$ set $\mathrm{N}(\sigma)=\mathrm{N}\left(n_{1}\right) \oplus \ldots \oplus \mathrm{N}\left(n_{r}\right)$. If in addition $a=\left(a_{1}, \ldots, a_{r}\right) \in\left(\mathscr{O}^{\times}\right)^{r}$ set

$$
\Phi(\sigma, a, q)=a_{1} \Phi\left(n_{1}, q\right) \oplus \ldots \oplus a_{r} \Phi\left(n_{r}, q\right) .
$$

Thus

$$
\Phi(\sigma, a, q) \mathrm{N}(\sigma) \Phi(\sigma, a, q)^{-1}=q \mathrm{~N}(\sigma) .
$$

We will write $\Phi(\sigma, q)$ for $\Phi(\sigma,(1, \ldots, 1), q)$.

Let $\mathrm{Nil}_{n}$ denote the space of $n \times n$ matrices with characteristic polynomial $\mathrm{X}^{n}$. It is an affine scheme over $\mathscr{O}$. (Brian Conrad has shown us a proof, due to N. Fakhruddin, that $\mathrm{Nil}_{n}$ is reduced, but we will not need this here.) For $\sigma=$ $\left\{n_{1}, \ldots, n_{r}\right\} \in \mathscr{P}_{n}$ we define a reduced closed subscheme $\operatorname{Nil}_{n}(\sigma) \subset \operatorname{Nil}_{n}$ to be the locus of matrices $\mathrm{N}$ for which all $n+1-d_{i}(\sigma)$ minors of $\mathrm{N}^{i}$ vanish for $i=$ $1, \ldots, n-1$ together with its reduced subscheme structure. Thus the matrix $\mathrm{N}(\sigma)$ defines an $\mathscr{O}$-point of $\operatorname{Nil}_{n}(\sigma)$. Note that $\operatorname{Nil}_{n}(\sigma) \supset \operatorname{Nil}_{n}\left(\sigma^{\prime}\right)$ if and only if $\sigma \leq \sigma^{\prime}$, and that

$$
\operatorname{Nil}_{n}(\sigma) \cap \operatorname{Nil}_{n}\left(\sigma^{\prime}\right)=\operatorname{Nil}_{n}\left(\sigma \vee \sigma^{\prime}\right)
$$

Thus the $\mathrm{Nil}_{n}(\sigma)$ with $\sigma \in \mathscr{P}_{n}$ form a stratification of $\mathrm{Nil}_{n}$. We will write

$$
\operatorname{Nil}_{n}(\sigma)^{0}=\operatorname{Nil}_{n}(\sigma)-\bigcup_{\sigma^{\prime}>\sigma} \operatorname{Nil}_{n}\left(\sigma^{\prime}\right) .
$$

If $\mathrm{L} / \mathscr{O}$ is a field then the L-points of $\operatorname{Nil}_{n}(\sigma)^{0}$ correspond to nilpotent matrices over $\mathrm{L}$ whose Jordan blocks have size $n_{1}, \ldots, n_{r}$, i.e. to $\mathrm{GL}_{n}(\mathrm{~L})$ conjugates of $\mathrm{N}(\sigma)$.

\section{Lemma 2.5.}

1. $\mathrm{Nil}_{n}(\sigma) / \mathscr{O}$ is integral, its fibres are irreducible and the open subscheme $\operatorname{Nil}_{n}(\sigma)^{0}$ is fibrewise dense in $\mathrm{Nil}_{n}(\sigma)$. Moreover $\mathrm{Nil}_{n}(\sigma)^{0}$ is smooth over $\mathrm{Spec} \mathscr{O}$ of relative dimension $n^{2}-\sum_{i=1}^{\infty}\left(d_{i}(\sigma)-d_{i-1}(\sigma)\right)^{2}$.

2. $\mathrm{Z}_{\mathrm{GL}_{n}}(\mathrm{~N}(\sigma))$ is a fibrewise dense open subset of the affine space associated to a free $\mathscr{O}$-module of rank $\sum_{i=1}^{\infty}\left(d_{i}(\sigma)-d_{i-1}(\sigma)\right)^{2}$.

3. Locally in the Zariski topology the universal matrix over $\operatorname{Nil}_{n}(\sigma)^{0}$ is conjugate, by a section of $\mathrm{GL}_{n}$, to $\mathrm{N}(\sigma)$.

Proof. - This is well known over a field. As we are unaware of a reference over a DVR, we give a proof here. We would like to thank Dennis Gaitsgory for help with this.

Let $\operatorname{Grass}(\sigma)$ denote the moduli space of increasing filtrations (denoted $\left.\left\{\text { Fil }_{j}\right\}_{j=0, \ldots, n}\right)$ of the free rank $n$ module $\mathscr{O}^{n}$ by locally direct summands for which Fil ${ }_{j}$ 
is locally free of $\operatorname{rank} d_{j}(\sigma)$. Then $\operatorname{Grass}(\sigma)$ is smooth over $\mathscr{O}$ of relative dimension $\left(n^{2}-\sum_{i}\left(d_{i}(\sigma)-d_{i-1}(\sigma)\right)^{2}\right) / 2$. It is integral with integral fibres. Let $\left\{\mathrm{Fil}_{j}^{\sigma}\right\}$ denote the standard filtration of $\mathscr{O}^{n}$, where $\mathrm{Fil}_{j}^{\sigma}$ consists of vectors whose last $n-d_{j}(\sigma)$ entries are zero. Locally in the Zariski topology on $\operatorname{Grass}(\sigma)$ there is a section $g$ of $\mathrm{GL}_{n}$ such that $\left\{g \mathrm{Fil}_{j}^{\sigma}\right\}$ is the universal filtration.

Let $\mathrm{Q}_{\sigma} \subset \mathrm{M}_{n \times n}$ denote the subscheme of matrices taking $\mathrm{Fil}_{j}^{\sigma}$ to $\mathrm{Fil}_{j-1}^{\sigma}$ for all $j$. Also let $\mathbf{Q}_{\sigma}^{0}$ denote the open subscheme of $\mathbf{Q}_{\sigma}$ defined as the locus of matrices which induce maps $\operatorname{gr}_{j}^{\sigma} \rightarrow \operatorname{gr}_{j-1}^{\sigma}$ of $\operatorname{rank} d_{j}(\sigma)-d_{j-1}(\sigma)=\operatorname{rkgr}_{j}^{\sigma}$. Thus $\mathrm{Q}_{\sigma}^{0} \subset \mathrm{Q}_{\sigma}$ is the complement of the intersection of the closed subschemes each defined by the vanishing of the determinant of a $\left(d_{j}(\sigma)-d_{j-1}(\sigma)\right) \times\left(d_{j}(\sigma)-d_{j-1}(\sigma)\right)$ minor of the $\left(d_{j}(\sigma)-d_{j-1}(\sigma)\right) \times\left(d_{j-1}(\sigma)-d_{j-2}(\sigma)\right)$ matrix representing the map $\operatorname{gr}_{j}^{\sigma} \rightarrow \operatorname{gr}_{j-1}^{\sigma}$. Let $\mathrm{Nil}_{n}^{\mathrm{Fil}}(\sigma)$ denote the moduli space of pairs $\left(\left\{\mathrm{Fil}_{j}\right\}, \mathrm{N}\right)$ where $\left\{\mathrm{Fil}_{j}\right\}$ is a filtration as above and where $\mathrm{N}$ is an endomorphism with $\mathrm{NFil}_{j} \subset \mathrm{Fil}_{j-1}$ for all $j$. Let $\mathrm{Nil}_{n}^{\text {Fil }}(\sigma)^{0}$ denote the open subspace where, for all $j$, the map $\mathrm{N}$ induces an isomorphism of $\mathrm{Fil}_{j+1} / \mathrm{Fil}_{j}$ with a locally direct summand of $\mathrm{Fil}_{j} / \mathrm{Fil}_{j-1}$. There are natural maps

$$
\mathrm{Nil}_{n}^{\mathrm{Fil}}(\sigma)^{0} \hookrightarrow \mathrm{Nil}_{n}^{\mathrm{Fil}}(\sigma) \longrightarrow \operatorname{Grass}(\sigma)
$$

Locally in the Zariski topology on $\operatorname{Grass}(\sigma)$ we have $\operatorname{Nil}_{n}^{\mathrm{Fil}}(\sigma) \cong \operatorname{Grass}(\sigma) \times \mathrm{Q}_{\sigma}$ and $\operatorname{Nil}_{n}^{\mathrm{Fil}}(\sigma)^{0} \cong \operatorname{Grass}(\sigma) \times \mathrm{Q}_{\sigma}^{0}$. (If on an open set $\mathrm{U} \subset \operatorname{Grass}(\sigma)$ there is a section $g$ of $\mathrm{GL}_{n}$ such that $\left\{g \mathrm{Fil}_{j}^{\sigma}\right\}$ is the universal filtration, then $\left.\mathrm{Nil}_{n}^{\mathrm{Fil}}(\sigma)\right|_{\mathrm{U}}$ is just $\mathrm{U} \times g \mathrm{Q}_{\sigma} g^{-1}$.) Thus $\mathrm{Nil}_{n}^{\mathrm{Fil}}(\sigma)$ is integral and smooth over $\mathscr{O}$ of relative dimension $n^{2}-\sum_{i=1}^{\infty}\left(d_{i}(\sigma)-\right.$ $\left.d_{i-1}(\sigma)\right)^{2}$. Also its fibres are integral and $\operatorname{Nil}_{n}^{\mathrm{Fil}}(\sigma)^{0}$ is fibrewise dense in $\operatorname{Nil}_{n}^{\mathrm{Fil}}(\sigma)$.

There is also a forgetful map $\mathrm{Nil}_{n}^{\mathrm{Fil}}(\sigma) \rightarrow \mathrm{Nil}_{n}$, which factors through $\mathrm{Nil}_{n}(\sigma)$. Using the valuative criterion one sees that this map is proper. Also it is surjective on points. Thus $\mathrm{Nil}_{n}(\sigma)$ is irreducible (and, as it is reduced by definition, also integral) and all its fibres are irreducible. Moreover $\mathrm{Nil}_{n}^{\mathrm{Fil}}(\sigma)^{0}$ maps isomorphically to $\operatorname{Nil}_{n}(\sigma)^{0}$. (To see that the map is an isomorphism, note that over $\operatorname{Nil}^{n}(\sigma)^{0}$ the sheaf $\operatorname{kerN}^{j}$ is a local direct summand of dimension $d_{j}(\sigma)$ of the free sheaf of rank $n$. The map $\mathrm{N} \mapsto\left(\left\{k \operatorname{ker} \mathrm{N}^{j}\right\}, \mathrm{N}\right)$ gives a two sided inverse to the forgetful map $\operatorname{Nil}_{n}^{\mathrm{Fil}}(\sigma)^{0} \rightarrow \mathrm{Nil}_{n}(\sigma)^{0}$.) Thus $\mathrm{Nil}_{n}(\sigma)^{0}$ is connected and smooth over $\mathscr{O}$ of relative dimension $n^{2}-\sum_{i=1}^{\infty}\left(d_{i}(\sigma)-d_{i-1}(\sigma)\right)^{2}$. Moreover $\operatorname{Nil}_{n}(\sigma)^{0}$ is fibrewise dense in $\operatorname{Nil}_{n}(\sigma)$.

The centraliser $\mathrm{Z}_{\mathrm{GL}_{n}}(\mathrm{~N}(\sigma))$ is the open subscheme of $\mathrm{Z}_{\mathrm{M}_{n \times n}}(\mathrm{~N}(\sigma))$ on which det is non-zero. The latter centraliser is the affine space associated to a finite, free $\mathscr{O}$-module. (The subscheme $\mathrm{Z}_{\mathrm{M}_{n \times n}}(\mathrm{~N}(\sigma)) \subset \mathrm{M}_{n \times n}$ is is the locus where some entries of the $n \times n$ matrix are 0 and certain others equal each other.) Moreover $\mathrm{Z}_{\mathrm{GL}_{n}}(\mathrm{~N}(\sigma))$ is fibrewise dense in $\mathrm{Z}_{\mathrm{M}_{n \times n}}(\mathrm{~N}(\sigma))$ because if one adds a generic multiple of the identity matrix to a point of $\mathrm{Z}_{\mathrm{M}_{n \times n}}(\mathrm{~N}(\sigma))$ one obtains a point of $\mathrm{Z}_{\mathrm{GL}_{n}}(\mathrm{~N}(\sigma))$. 
Over $\mathrm{K}$ the natural map

$$
\mathrm{GL}_{n} / \mathrm{Z}_{\mathrm{GL}_{n}}(\mathrm{~N}(\sigma)) \longrightarrow \mathrm{Nil}_{n}(\sigma)^{0}
$$

is a bijection on points and hence an isomorphism. Thus $\mathrm{Z}_{\mathrm{M}_{n \times n}}(\mathrm{~N}(\sigma))$ has dimension $\sum_{i=1}^{\infty}\left(d_{i}(\sigma)-d_{i-1}(\sigma)\right)^{2}$.

Zariski locally on $\mathrm{Nil}_{n}(\sigma)^{0}=\mathrm{Nil}_{n}^{\mathrm{Fil}}(\sigma)^{0}$ we may assume that each graded piece of the universal filtration is free. Choose a basis of the free rank $n$ module as follows. Choose a basis of the top graded piece of the universal filtration and lift these elements to elements $f_{1}, \ldots, f_{a}$ of the free rank $n$ module. Add to these all non-zero images of these elements under powers of $\mathrm{N}$. Now extend $\mathrm{N} f_{1}, \ldots, \mathrm{N} f_{a}$ to a basis of the penultimate graded piece of the universal filtration and lift the new basis elements to $f_{a+1}, \ldots, f_{a+b}$. Add these and all their non-zero images under powers of $\mathrm{N}$ to our putative basis and continue in this way. We will end up with (a permutation of) a basis with respect to which $\mathrm{N}$ has matrix $\mathrm{N}(\sigma)$. Thus we can find a section $g$ of $\mathrm{GL}_{n}$ over this open set such that $\mathrm{N}=g \mathrm{~N}(\sigma) g^{-1}$.

Next we turn to a couple of results about complete local rings.

Lemma 2.6. - Suppose that $\mathrm{A}$ is a complete noetherian local $\mathscr{O}$-algebra (so that $\lambda \mathrm{A}$ is contained in the maximal ideal $\mathfrak{m}$ of $\mathrm{A})$.

1. Suppose that $l$ is not nilpotent in $\mathrm{A}$ and that $\wp$ is a maximal ideal of $\mathrm{A}[1 / l]$. Then $\mathrm{A}[1 / l] / \wp$ is a finite field extension of $\mathrm{K}$ and $\mathrm{A} / \wp^{c}$ is a finite free $\mathscr{O}$-module (and hence an order in $\mathrm{A}[1 / l] / \wp)$.

2. Any prime ideal of $\mathrm{A}[1 / l]$ equals the intersection of the maximal ideals containing it.

Proof. - The first part follows by the proof of Lemma 5.1.1 of [BM]. (That is, we may suppose that $\wp^{c}=(0) \subset \mathrm{A}$. Then $\mathrm{A}$ is an integral domain which is flat over $\mathscr{O}$, and $\mathrm{A}[1 / l]$ is a field. Theorem 15.3 of $[\mathrm{M}]$ tells us that the Krull dimension of $\mathrm{A} / \lambda$ is also zero, so that $\mathrm{A} / \lambda$ is a finite $\mathscr{O}$-module. It follows that A is also a finite $\mathscr{O}$-module, from which the desired result follows.) The second part is a special case of Corollary 10.5.8 of [EGA].

Lemma 2.7. - Let $\mathrm{X} / \mathrm{Spec} \mathscr{O}$ be a scheme of finite type. Write $\mathrm{X}_{1}, \ldots, \mathrm{X}_{r}$ for the irreducible components of $\mathrm{X}$ with their reduced subscheme structure. Assume that the distinct irreducible components of $\mathrm{X} \times_{\text {Spec } \mathscr{O}}$ Spec $k$ are the $\mathrm{X}_{i} \times_{\text {Spec } \mathscr{O}}$ Spec $k$ and that each is (nonempty and) generically reduced. Also assume that all the $\mathrm{X}_{i} \times_{\operatorname{Spec} \mathscr{O}} \mathrm{Spec} \mathrm{K}$ are non-empty and have the same dimension $d$.

Let $x$ be a closed point of $\mathrm{X} \times{ }_{\mathrm{Spec} \mathscr{O}} \mathrm{Spec} k$. Then

- no minimal prime of the completion $\mathscr{O}_{\mathrm{X}, x}^{\wedge}$ contains $\lambda \mathscr{O}_{\mathrm{X}, x}^{\wedge}$;

- all maximal chains of prime ideals in $\mathscr{O}_{\mathrm{X}, x}^{\wedge}$ have length $d+2$; 
- every minimal prime of $\mathscr{O}_{\mathrm{X}, x}$ is contained in a prime minimal over $\lambda \mathscr{O}_{\mathrm{X}, x}^{\wedge}$; and

- every prime of $\mathscr{O}_{\mathrm{X}, x}$ which is minimal over $\lambda \mathscr{O}_{\mathrm{X}, x}$ contains a unique minimal prime of $\mathscr{O}_{\mathrm{X}, x}^{\wedge}$.

Proof. - We may assume that $\mathrm{X}$ is reduced. Then $\mathrm{X}$ is flat over Spec $\mathscr{O}$ (because it is reduced and all its generic points have characteristic 0) and hence $\mathscr{O}_{\mathrm{X}, x}^{\wedge}$ is flat over $\mathscr{O}$, i.e. has no $\lambda$-torsion. Any minimal prime of $\mathscr{O}_{\mathrm{X}, x}^{\wedge}$ is also an associated prime of $\mathscr{O}_{\mathrm{X}, x}^{\wedge}$ and so can not contain $\lambda \mathscr{O}_{\mathrm{X}, x}^{\wedge}$. (Else $\mathscr{O}_{\mathrm{X}, x}^{\wedge}$ would have $\lambda$-torsion.)

Let $\tilde{\mathrm{X}}$ denote the normalisation of $\mathrm{X}$ and let $\tilde{\mathrm{X}}_{i}$ denote the pull back of $\mathrm{X}_{i}$ to $\tilde{\mathrm{X}}$. Then $\widetilde{\mathrm{X}}_{i}$ is simply the normalisation of $\mathrm{X}_{i}$ and $\widetilde{\mathrm{X}}$ is the disjoint union of the $\widetilde{\mathrm{X}}_{i}$. As $\mathrm{X}$ is excellent each $\widetilde{\mathrm{X}}_{i}$ is finite over $\mathrm{X}_{i}$. We see that each $\widetilde{\mathrm{X}}_{i} \times_{\text {Spec }}$ Spec $\mathrm{K}$ also has dimension $d$. By [EGA] IV.13.1.3 and [EGA] IV.14.3.10 we conclude that each $\mathrm{X}_{i} \times_{\text {Spec } \mathscr{O}}$ Spec $k$ and each irreducible component of each $\widetilde{\mathrm{X}}_{i} \times_{\text {Spec } \mathscr{O}}$ Spec $k$ also has dimension $d$. (We thank Brian Conrad for this reference.) Thus each irreducible component of $\widetilde{\mathrm{X}}_{i} \times_{\mathrm{Spec} \mathscr{O}}$ Spec $k$ dominates $\mathrm{X}_{i} \times_{\text {Spec } \mathscr{O}}$ Spec $k$. Let $\mathrm{Q}$ denote the coherent sheaf on $\mathrm{X}$ which is the quotient of the push forward of $\mathscr{O}_{\widetilde{\mathrm{X}}}$ by $\mathscr{O}_{\mathrm{X}}$.

Let $\bar{\eta}_{i}$ denote the generic point of $\mathrm{X}_{i} \times_{\mathrm{Spec} \mathscr{O}}$ Spec $k$. Then $\mathscr{O}_{\mathrm{X}_{i}, \bar{\eta}_{i}} / \lambda$ is a field and so $\mathscr{O}_{\mathrm{X}_{i}, \bar{\eta}_{i}}=\mathscr{O}_{\mathrm{X}, \bar{\eta}}$ is a DVR with maximal ideal generated by $\lambda$. But $\mathscr{O}_{\widetilde{\mathrm{X}}_{i}, \bar{\eta}_{i}}$ is just the integral closure $\mathscr{O}_{\mathrm{X}_{i}, \bar{\eta}_{i}}$, so that

$$
\mathscr{O}_{\widetilde{\mathrm{X}}_{i}, \bar{\eta}_{i}}=\mathscr{O}_{\mathrm{X}_{i}, \bar{\eta}_{i}}=\mathscr{O}_{\mathrm{X}, \bar{\eta}_{i}} .
$$

In particular there is a unique point of $\tilde{\mathrm{X}}_{i}$ above $\bar{\eta}_{i}$ and we conclude that $\widetilde{\mathrm{X}}_{i} \times{ }_{\mathrm{Spec} \mathscr{O}}$ Spec $k$ is irreducible. Moreover we see that $Q_{\bar{\eta}_{i}}=(0)$.

As $\tilde{\mathrm{X}} / \mathrm{X}$ is finite we have

$$
\mathscr{O}_{\widetilde{\mathrm{X}}, x}^{\wedge}=\bigoplus_{i, y} \mathscr{O}_{\widehat{\mathrm{X}}_{i, y}}
$$

where $y$ runs over the points of $\tilde{\mathrm{X}}_{i}$ above $x$. Thus there is an exact sequence

$$
(0) \longrightarrow \mathscr{O}_{\mathrm{X}, x}^{\wedge} \longrightarrow \bigoplus_{i, y} \mathscr{O}_{\tilde{\mathrm{X}}_{i, y}}^{\wedge} \longrightarrow \mathrm{Q}_{x}^{\wedge} \longrightarrow(0)
$$

As $\tilde{\mathrm{X}}_{i}$ is excellent, reduced and normal, we deduce that each $\mathscr{O}_{\tilde{\mathrm{X}}_{i, y}}$ is reduced and integrally closed and hence (as it is also local) an integral domain. Let $\mathfrak{p}_{i, y}$ denote the prime ideal which is the kernel of the map $\mathscr{O}_{\mathrm{X}, x}^{\wedge} \rightarrow \mathscr{O}_{\tilde{\mathrm{X}}_{i, y}}^{\wedge}$. If $\wp$ is a prime of $\mathscr{O}_{\mathrm{X}, x}^{\wedge}$ above a generic point of some $\mathrm{X}_{i}$ or of some $\mathrm{X}_{i} \times_{\text {Spec } \mathscr{O}}$ Spec $k$ then $\left(\mathrm{Q}_{x}^{\wedge}\right)_{\wp}=(0)$ and so

$$
\left(\mathscr{O}_{\mathrm{X}, x}^{\wedge}\right)_{\wp} \stackrel{\sim}{\longrightarrow} \bigoplus_{i, y}\left(\mathscr{O}_{\tilde{\mathrm{X}}_{i, y}}^{\wedge}\right)_{\wp} .
$$


(The module $\mathbf{Q}_{x}$ is killed by some element $f \in \mathscr{O}_{\mathbf{X}, x}-\wp$, and $f$ will also kill $\mathbf{Q}_{x}^{\wedge}$.) As $\left(\mathscr{O}_{\mathrm{X}, x}^{\wedge}\right)_{\wp}$ is local we see that $\wp$ contains $\mathfrak{p}_{i, y}$ for a unique pair $(i, y)$.

Suppose first that $\wp$ is a minimal prime of $\mathscr{O}_{\mathbf{X}, x}^{\wedge}$. Then $\wp$ lies above the generic point of one of the $\mathrm{X}_{i}$ (by the going down theorem, because $\mathscr{O}_{\mathrm{X}, x}^{\wedge}$ is flat over $\left.\mathscr{O}_{\mathrm{X}, x}\right)$. Thus $\wp \supset \mathfrak{p}_{i, y}$ for a unique pair $(i, y)$. As each $\mathfrak{p}_{i, y}$ contains some minimal prime ideal of $\mathscr{O}_{\mathrm{X}, x}$ we deduce that the $\mathfrak{p}_{i, y}$ are minimal, distinct and in fact exhaust the minimal primes of $\mathscr{O}_{\mathrm{X}, x}^{\wedge}$.

As $\mathscr{O}_{\tilde{\mathrm{X}}_{i, y}}^{\wedge} \supset \mathscr{O}_{\mathrm{X}, x}^{\wedge} / \mathfrak{p}_{i, y}$ is a finite extension of domains, they both have the same dimension (see Section 4.4 of [E]). On the other hand $\mathscr{O}_{\hat{\mathrm{X}}_{i, y}}$ has the same dimension as $\mathscr{O}_{\widetilde{\mathrm{X}}_{i, y}}$ (Corollary 12.5 of $[\mathrm{E}]$ ), which equals one plus $\operatorname{dim} \mathscr{O}_{\widetilde{\mathrm{X}}_{i} \times \operatorname{Spec} k, y}$ (Theorem 10.10 of $[\mathrm{E}]$ ), which in turn equals $1+\operatorname{dim}\left(\tilde{\mathrm{X}}_{i} \times{ }_{\operatorname{Spec} \mathscr{O}} \operatorname{Spec} k\right.$ ) (Theorem A of Section 13.1 of [E]). We have already seem that this number is just $1+d$. As $\mathscr{O}_{\tilde{\mathrm{X}}_{i, y}}$ is catenary, the second part of the lemma follows.

Suppose that $\wp$ is a prime of $\mathscr{O}_{\mathrm{X}, x}^{\wedge}$ which is minimal over $\left(\lambda, \mathfrak{p}_{i, y}\right)$. We claim that $\wp$ is a minimal prime over $\lambda \mathscr{O}_{\mathrm{X}, x}^{\wedge}$, from which the third part of the lemma would follow. If not we can find a prime $\mathfrak{q}$ with $\wp \supset \mathfrak{q} \supset \lambda \mathscr{O}_{\mathrm{X}}^{\wedge}, x$, but $\mathfrak{q} \neq \wp$. Then $\mathfrak{q}$ must contain a minimal prime $\mathfrak{p}_{i^{\prime}, y^{\prime}} \neq \mathfrak{p}_{i, y}$. It would follow that

$$
\operatorname{dim} \mathscr{O}_{\mathbf{X}, x}^{\wedge} / \mathfrak{p}_{i, y}=1+\operatorname{dim} \mathscr{O}_{\mathbf{X}, x}^{\wedge} / \wp>1+\operatorname{dim} \mathscr{O}_{\mathbf{X}, x}^{\wedge} / \mathfrak{p}_{i^{\prime}, y^{\prime}}
$$

(see Theorem 10.1 of [E]), which would contradict the second part of this lemma.

Suppose now that $\wp$ is any prime of $\mathscr{O}_{\mathrm{X}, x}^{\wedge}$ minimal over $\lambda \mathscr{O}_{\mathrm{X}, x}^{\wedge}$. As the quotient $\mathscr{O}_{\mathrm{X}, x}^{\wedge} / \lambda \mathscr{O}_{\mathrm{X}, x}^{\wedge}=\mathscr{O}_{\mathrm{X} \times \mathrm{Spec} k, x}^{\wedge}$ is flat over $\mathscr{O}_{\mathrm{X} \times \operatorname{Spec} k, x}$ we deduce (by the going down theorem) that $\wp$ lies over the generic point of one of the $\mathrm{X}_{i} \times_{\operatorname{Spec} \mathscr{O}}$ Spec $k$. Thus $\wp$ contains a unique $\mathfrak{p}_{i, y}$, i.e. a unique minimal prime of $\mathscr{O}_{\mathbf{X}, x}^{\wedge}$. This proves the fourth part of the lemma.

\section{Some more local deformation problems}

This section may be considered a continuation of Section 2.4 of [CHT]. We will study some further local deformation problems which are of key importance to the main strategy of this paper.

Fix a positive integer $n$ and a prime number $l>n$. Let $\mathrm{K}$ denote a finite extension of $\mathbf{Q}_{l}$ with ring of integers $\mathscr{O}$, maximal ideal $\lambda$ and residue field $k$. Let $p \neq l$ be a second prime and $\mathrm{F}_{\widetilde{v}}$ be a finite extension of $\mathbf{Q}_{p}$ with residue field $k(\tilde{v})$. We will suppose that

$$
\# k(\widetilde{v}) \equiv 1 \bmod l .
$$

Further we will let

$$
\bar{r}: \mathrm{G}_{\mathrm{F}_{\tilde{v}}} \longrightarrow\left\{1_{n}\right\} \subset \mathrm{GL}_{n}(k)
$$

denote the trivial representation. 
We will let $\mathrm{G}_{\mathrm{F}_{\widetilde{v}}}$ denote the absolute Galois group of $\mathrm{F}_{\widetilde{v}}$. Also $\mathrm{I}_{\widetilde{F}}$ will denote its inertia subgroup and Frob $_{\widetilde{v}}$ the geometric Frobenius in $\mathrm{G}_{\mathrm{F}_{\tilde{v}}} / \mathrm{I}_{\mathrm{F}_{\tilde{v}}}$. Choose a lifting $\phi_{\widetilde{v}} \in \mathrm{G}_{\mathrm{F}_{\widetilde{v}}}$ of Frob $_{\widetilde{v}}^{-1}$. In addition $\mathscr{C}_{\mathscr{O}}^{f}$ will denote the category of Artinian local $\mathscr{O}$-algebras with residue field $k$ and $\mathscr{C}_{\mathscr{O}}$ the category of topological local $\mathscr{O}$-algebras which are isomorphic to inverse limits of objects in $\mathscr{C}_{\mathscr{O}}^{f}$.

Suppose that

$$
\chi_{v, 1}, \ldots, \chi_{v, n}: \mathrm{G}_{\widetilde{F}_{\tilde{v}}} \rightarrow(1+\lambda) \subset \mathscr{O}^{\times}
$$

are continuous characters. Thus

$$
\left.\chi_{v, i}\right|_{\mathrm{I}_{\widetilde{v}}} ^{\# k(\widetilde{v})-1}=1 .
$$

We will let $\mathscr{D}_{v}^{\left(\chi_{v, 1}, \ldots, \chi_{v, n}\right)}$ denote the set of liftings $r$ of $\bar{r}$ to objects of $\mathscr{C}_{\mathscr{O}}$ such that for all $\sigma \in \mathrm{I}_{\mathrm{F}_{\widetilde{v}}}$

$$
\operatorname{char}_{r(\sigma)}(\mathrm{X})=\prod_{i=1}^{n}\left(\mathrm{X}-\chi_{i, j}(\sigma)\right) .
$$

The collection $\mathscr{D}_{v}^{\left(\chi_{v, 1}, \ldots, \chi_{v, n}\right)}$ is a local deformation problem in the sense of Definition 2.2.2 of [CHT]. Let $\mathscr{I}_{v}\left(\chi_{v, 1}, \ldots, \chi_{v, n}\right)$ denote the corresponding ideal of the universal local lifting ring $\mathrm{R}_{v}^{\text {loc }}$ for $\bar{r}$. We will also let $\mathscr{D}_{v}^{\text {Stein, },} \subset \mathscr{D}_{v}^{(1,1, \ldots, 1)}$ denote the subset consisting of liftings $r$ such that

$$
\operatorname{char}_{r\left(\phi_{\widetilde{v}}\right.}(\mathrm{X}) \in \operatorname{Pol}_{n}(\{n\}, \# k(v)) .
$$

It is again local deformation problem and we will write $\mathscr{I}_{v}^{\text {Stein, } 1}$ for the corresponding ideal of $\mathrm{R}_{v}^{\text {loc }}$. Then we let $\mathscr{I}_{v}^{\text {Stein }} \supset \mathscr{J}_{v}^{\text {Stein,l }}$ denote the preimage in $\mathrm{R}_{v}^{\text {loc }}$ of the ideal of $\mathrm{R}_{v}^{\text {loc }} / \mathscr{I}_{v}^{\text {Stein, } 1}$ consisting of all elements killed by a power of $\lambda$. We also let $\mathscr{D}^{\text {Stein }}$ denote the corresponding deformation problem. Note that $\mathrm{R}_{v}^{\text {loc }} / \mathscr{I}_{v}^{\text {Stein }}$ is by definition flat over $\mathscr{O}$. (It seems likely that $\mathscr{D}^{\text {Stein }}=\mathscr{D}^{\text {Stein, } 1}$ and $\mathscr{I}_{v}^{\text {Stein }}=\mathscr{J}_{v}^{\text {Stein, }, 1}$. However we won't need this, so we haven't tried to prove it.) The sole purpose of this (and most of the previous) section is to prove the following proposition.

\section{Proposition 3.1.}

1. If all the $\left.\chi_{v, i}\right|_{\mathrm{I}_{\tilde{v}}}$ are distinct characters then the spectrum $\operatorname{Spec} \mathbf{R}_{v}^{\operatorname{loc}} / \mathscr{I}_{v}\left(\chi_{v, 1}, \ldots, \chi_{v, n}\right)$ is irreducible and its generic point has characteristic zero. Moreover $\mathbf{R}_{v}^{\mathrm{loc}} / \mathscr{I}_{v}^{\left(\chi_{v, 1}, \ldots, \chi_{v, n}\right)}$ has Krull dimension $n^{2}+1$.

2. $\mathbf{R}_{v}^{\text {loc }} /\left(\lambda, \mathscr{I}_{v}^{\left(\chi_{v, 1}, \ldots, \chi_{v, n}\right)}\right)=\mathrm{R}_{v}^{\text {loc }} /\left(\lambda, \mathscr{I}_{v}^{(1,1, \ldots, 1)}\right)$.

3. All irreducible components of $\operatorname{Spec} \mathrm{R}_{v}^{\mathrm{loc}} / \mathscr{I}_{v}^{(1,1, \ldots, 1)}$ have dimension $n^{2}+1$ and their generic points all have characteristic zero. Moreover every prime of $\mathbf{R}_{v}^{\text {loc }} / \mathscr{I}_{v}^{(1,1, \ldots, 1)}$ which is minimal over $\lambda\left(\mathrm{R}_{v}^{\text {loc }} / \mathscr{I}_{v}^{(1,1, \ldots, 1)}\right)$ contains a unique minimal prime.

4. Spec $\mathrm{R}_{v}^{\text {loc }} / \mathscr{I}_{v}^{\text {Stein }}$ is irreducible and the generic point has characteristic zero. Moreover $\mathrm{R}_{v}^{\text {loc }} / \mathscr{I}_{v}^{\text {Stein }}$ has Krull dimension $n^{2}+1$. 
Note that any lifting of $\bar{r}$ to an Artinian local $\mathscr{O}$-algebra will factor through

$$
\mathrm{T}_{\mathrm{F}_{\tilde{v}}}=\mathrm{G}_{\mathrm{F}_{\widetilde{v}}} / \mathrm{P}_{\mathrm{F}_{\tilde{v}}},
$$

where $\mathrm{P}_{\mathrm{F}_{\tilde{\nu}}}$ denotes the kernel of any (and hence all) non-trivial maps from $\mathrm{I}_{\widetilde{\Gamma}}$ to $\mathbf{Z}_{l}$. Fix a topological generator $\sigma_{\widetilde{v}}$ of $\mathrm{I}_{\mathrm{F}_{\tilde{v}}} / \mathrm{P}_{\mathrm{F}_{\tilde{v}}}$. Then $\mathrm{T}_{\mathrm{F}_{\widetilde{v}}}$ is topologically generated by $\sigma_{\widetilde{v}}$ and $\phi_{\widetilde{v}}$ subject only to $\sigma_{\widetilde{v}}$ generating a pro-l-group and to

$$
\phi_{\widetilde{v}} \sigma_{\widetilde{v}} \phi_{\widetilde{v}}^{-1}=\sigma_{\widetilde{v}}^{\# k(v)} .
$$

If $\mathrm{P} \in \mathscr{O}[\mathrm{X}]$ is a monic polynomial of degree $n$ and if $q$ is a positive integer not divisible by $l$ we will let $\mathscr{M}(\mathrm{P}, q) / \mathscr{O}$ denote the moduli space of pairs of $n \times n$-matrices $(\Phi, \Sigma)$ such that

- $\Phi$ is invertible;

$-\Sigma$ has characteristic polynomial P;

- $\Phi \Sigma \Phi^{-1}=\Sigma^{q}$.

Thus $\mathscr{M}(\mathrm{P}, q)$ is an affine scheme over $\mathscr{O}$. Also write $\mathscr{M}_{n}^{\text {Stein }}(q)$ for the closed subscheme of $\mathscr{M}\left((\mathrm{X}-1)^{n}, q\right)$ defined by

$$
\operatorname{char}_{\Phi}(\mathrm{X}) \in \operatorname{Pol}_{n}(\{n\}, q) .
$$

Then $\mathbf{R}_{v}^{\text {loc }} / \mathscr{I}_{v}\left(\chi_{v, 1}, \ldots, x_{v, n}\right)$ (resp. $\left.\mathbf{R}_{v}^{\text {loc }} / \mathscr{I}_{v}^{\text {Stein }}\right)$ is the formal completion of the structure sheaf of

$$
\mathscr{M}\left(\prod_{i=1}^{n}\left(\mathrm{X}-\chi_{v, i}\left(\sigma_{\tilde{v}}\right)\right), \# k(v)\right)
$$

(resp. $\left.\mathscr{M}_{n}^{\text {Stein }}(\# k(v))\right)$ at the closed point $\left(1_{n}, 1_{n}\right)$ (in the special fibre). We will first prove the following global result.

Lemma 3.2. - Let $q$ be a positive integer with $q \equiv 1 \bmod l$.

1. Let $\mathscr{M}_{i}$ denote the irreducible components of $\mathscr{M}\left((\mathrm{X}-1)^{n}, q\right)$ with their reduced subscheme structure. Then each $\mathscr{M}_{i} \times_{\mathrm{Spec} \mathscr{O}} \mathrm{Spec} \mathrm{K}$ is non-empty of dimension $n^{2}$. Moreover the distinct irreducible components of $\mathscr{M}\left((\mathrm{X}-1)^{n}, q\right) \times_{\mathrm{Spec} \mathscr{O}} \mathrm{Spec} k$ are the $\mathscr{M}_{i} \times_{\mathrm{Spec} O} \mathcal{O}$ Spec $k$ and each is (non-empty and) generically reduced.

2. Suppose $\alpha_{1}, \ldots, \alpha_{n}$ are distinct $(q-1)^{\text {st }}$ roots of unity in $1+\lambda \subset \mathscr{O}$. (If $q=1$ we allow any $\alpha_{1}, \ldots, \alpha_{n} \in 1+\lambda$, not necessarily distinct.) Then $\mathscr{M}\left(\prod_{i=1}^{n}\left(\mathrm{X}-\alpha_{i}\right), q\right)^{\mathrm{red}}$ is flat over $\mathscr{O}$.

Proof. - We first give another model for $\mathscr{M}\left((\mathrm{X}-1)^{n}, q\right)$. To this end let $\mathscr{N}(q) / \mathscr{O}$ denote the moduli space of pairs of $n \times n$-matrices $(\Phi, \mathrm{N})$ such that 
- $\Phi$ is invertible;

- N has characteristic polynomial $\mathrm{X}^{n}$;

- $\Phi \mathrm{N} \Phi^{-1}=q \mathrm{~N}$.

Then $\mathscr{N}(q)$ is an affine scheme over $\mathscr{O}$. It follows from Lemma 2.4 that

$$
\mathscr{M}\left((\mathrm{X}-1)^{n}, q\right) \cong \mathscr{N}(q) .
$$

The map sends $(\Phi, \Sigma)$ to $(\Phi, \log \Sigma)$. (In the original version of the paper we only showed that the reduced subschemes were isomorphic, and this is all we actually need in the sequel. We thank Brian Conrad for suggesting we include this slightly stronger result and for showing us a proof due to N. Fakhruddin. This is not, however, the proof we have presented here.)

There are natural maps

$$
\mathscr{N}(q) \longrightarrow \mathrm{Nil}_{n}
$$

(taking $(\Phi, \mathrm{N})$ to $\mathrm{N})$ and

$$
\mathscr{N}(q) \longrightarrow \operatorname{Pol}_{n}
$$

(taking $(\Phi, \mathrm{N})$ to the characteristic polynomial of $\Phi)$. For $\sigma \in \mathscr{P}_{n}$ let

- $\mathscr{N}(q, \sigma)^{0}$ denote the locally closed preimage of $\mathrm{Nil}_{n}(\sigma)^{0}$ under the first of these maps;

- $\mathscr{N}(q, \sigma)$ denote the reduced subscheme of the closure of $\mathscr{N}(q, \sigma)^{0}$ in $\mathscr{N}(q)$

- and $\mathscr{N}(q, \sigma)^{\prime}$ denote the reduced subscheme of the intersection of the preimage under the first map of $\mathrm{Nil}_{n}(\sigma)$ with the preimage under the second map of $\operatorname{Pol}_{n}(\sigma, q)$.

Suppose that $\mathrm{L}$ is a field and that $(\Phi, \mathrm{N}) \in \mathrm{M}_{n \times n}(\mathrm{~L})^{2}$ defines an L-point of $\mathscr{N}(q, \sigma)^{0}$. Then $\Phi$ acts on $\operatorname{ker} \mathrm{N}^{i} / \operatorname{ker} \mathrm{N}^{i-1}$ for $i=1, \ldots, n$ and

$$
\operatorname{char}_{\left.\Phi\right|_{\left(\operatorname{ker~N} N^{i+1} / \operatorname{ker~N} N^{i}\right.}}(\mathrm{X}) \mid \operatorname{char}_{\left.\Phi\right|_{\left(\operatorname{ker} N^{i} / \operatorname{ker} N^{i-1}\right)}}(q \mathrm{X}) .
$$

Thus $\operatorname{char}_{\Phi}(\mathrm{X}) \in \operatorname{Pol}(\sigma, q)(\mathrm{L})$. It follows that

$$
\mathscr{N}(q, \sigma)^{\prime} \supset \mathscr{N}(q, \sigma) \supset\left(\mathscr{N}(q, \sigma)^{0}\right)^{\mathrm{red}} .
$$

Locally in the Zariski topology the map

$$
\mathscr{N}(q, \sigma)^{0} \longrightarrow \operatorname{Nil}_{n}(\sigma)^{0}
$$

is isomorphic to the projection

$$
\mathrm{Nil}_{n}(\sigma)^{0} \times \mathrm{Z}_{\mathrm{GL}_{n}}(\mathrm{~N}(\sigma)) \longrightarrow \operatorname{Nil}_{n}(\sigma)^{0} .
$$


(If over an open subset $\mathrm{U} \subset \mathrm{Nil}_{n}(\sigma)^{0}$ the universal matrix is $g \mathrm{~N}(\sigma) g^{-1}$ with $g$ a section of $\mathrm{GL}_{n}$ over $\mathrm{U}$, then the preimage of $\mathrm{U}$ in $\mathscr{N}(q, \sigma)^{0}$ is just $\mathrm{U} \times g \Phi(\sigma, q) \mathrm{Z}_{\mathrm{GL}_{n}}(\mathrm{~N}(\sigma)) g^{-1}$.) In particular $\mathscr{N}(q, \sigma)^{0}$ is smooth over $\mathscr{O}$ and fibrewise integral of pure relative dimension $n^{2}$. Suppose that $a_{1}, \ldots, a_{r} \in \bar{k}^{\times}$are such that $a_{i} q^{j} \neq a_{i^{\prime}} q^{j^{\prime}}$ for $i \neq i^{\prime}$ and $0 \leq j \leq n_{i}$ and $0 \leq j^{\prime} \leq n_{i^{\prime}}$. Then $(\Phi(\sigma, a, q), \mathrm{N}(\sigma))$ is a point of

$$
\left(\mathscr{N}(q, \sigma)-\bigcup_{\sigma^{\prime} \neq \sigma} \mathscr{N}\left(q, \sigma^{\prime}\right)^{\prime}\right)(\bar{k}) \subset\left(\mathscr{N}(q, \sigma)-\bigcup_{\sigma^{\prime} \neq \sigma} \mathscr{N}\left(q, \sigma^{\prime}\right)\right)(\bar{k}) .
$$

We conclude that the $\mathscr{N}(q, \sigma)\left(\right.$ resp. $\left.(\mathscr{N}(q, \sigma) \times \operatorname{Spec} k)^{\text {red }}\right)$ are the reduced irreducible components of $\mathscr{N}(q)$ (resp. $\mathscr{N}(q) \times \operatorname{Spec} k)$. Each irreducible component of $\mathscr{N}(q) \times$ Spec $k$ is contained in a unique irreducible component of $\mathscr{N}(q)$. The first part of the lemma follows.

Notice that if $\alpha_{1}, \ldots, \alpha_{n} \in 1+\lambda$ then

$$
\begin{aligned}
\mathscr{M}\left(\prod_{i=1}^{n}\left(\mathrm{X}-\alpha_{i}\right), 1\right) \times_{\mathrm{Spec} \mathscr{O}} \operatorname{Spec} k & =\mathscr{M}\left((\mathrm{X}-1)^{n}, 1\right) \times_{\mathrm{Spec} \mathscr{O}} \operatorname{Spec} k \\
& \cong \mathscr{N}(q) \times_{\mathrm{Spec} \mathscr{O}} \operatorname{Spec} k .
\end{aligned}
$$

Over the generic point of $\mathscr{N}(q, \sigma) \times_{\mathrm{Spec} \mathscr{O}} \operatorname{Spec} k$ the pair $(\Phi, \mathrm{N})$ is of the form $\left(\mathrm{A} \Phi(\sigma, a, q) \mathrm{A}^{-1}, \mathrm{AN}(\sigma) \mathrm{A}^{-1}\right)$ for some $a$ and $\mathrm{A}$. Thus over the generic point of the corresponding component of $\mathscr{M}\left(\prod_{i=1}^{n}\left(\mathrm{X}-\alpha_{i}\right), 1\right) \times_{\mathrm{Spec} \mathscr{O}} \operatorname{Spec} k$ the pair $(\Phi, \underset{\widetilde{\mathrm{A}}}{\Sigma}$ is of the form $\left(\mathrm{A} \Phi(\sigma, a, 1) \mathrm{A}^{-1}, \mathrm{~A}(\exp \mathrm{N}(\sigma)) \mathrm{A}^{-1}\right)$. Choosing liftings $\tilde{a}$ of $a$ and $\widetilde{\mathrm{A}}$ of A to characteristic 0 we can lift this to the characteristic zero pair

$$
\left(\widetilde{\mathrm{A}} \Phi(\sigma, \widetilde{a}, 1) \widetilde{\mathrm{A}}^{-1}, \widetilde{\mathrm{A}} \operatorname{diag}\left(\alpha_{1}, \ldots, \alpha_{n}\right)(\exp \mathrm{N}(\sigma)) \widetilde{\mathrm{A}}^{-1}\right) .
$$

Thus all generic points of $\mathscr{M}\left(\prod_{i=1}^{n}\left(\mathrm{X}-\alpha_{i}\right), 1\right)$ have characteristic zero and $\mathscr{M}\left(\prod_{i=1}^{n}\left(\mathrm{X}-\alpha_{i}\right), 1\right)^{\text {red }}$ is flat over $\mathscr{O}$.

If $\alpha_{1}, \ldots, \alpha_{n}$ are distinct $(q-1)^{s t}$ roots of unity in $\mathscr{O}$ and if $\Sigma$ is an $n \times n$ matrix with

$$
\operatorname{char}_{\Sigma}(\mathrm{X})=\prod_{i=1}^{n}\left(\mathrm{X}-\alpha_{i}\right) \mid\left(\mathrm{X}^{q-1}-1\right)
$$

then $\Sigma^{q-1}=1_{n}$. Thus

$$
\mathscr{M}\left(\prod_{i=1}^{n}\left(\mathrm{X}-\alpha_{i}\right), q\right)=\mathscr{M}\left(\prod_{i=1}^{n}\left(\mathrm{X}-\alpha_{i}\right), 1\right)
$$

and the second part of the lemma follows. 
Lemma 3.3. - Suppose that all the $\left.\chi_{v, i}\right|_{\mathrm{I}_{\widetilde{v}}}$ are distinct characters. Then the formal completion of $\left(\mathrm{R}_{v}^{\mathrm{loc}} / \mathscr{I}_{v}^{\left(\chi_{v, 1}, \ldots, \chi_{v, n}\right)}\right)[1 / l]$ at any maximal ideal is formally smooth over $\mathrm{K}$. Similarly the formal completion of

$$
\left(\mathrm{R}_{v}^{\text {loc }} / \mathscr{J}_{v}^{\text {Stein }}\right)[1 / l]=\left(\mathrm{R}_{v}^{\text {loc }} / \mathscr{I}_{v}^{\text {Stein, } 1}\right)[1 / l]
$$

at any maximal ideal is formally smooth over $\mathrm{K}$.

Proof. - Consider first the case of

$$
\mathrm{R}=\mathrm{R}_{v}^{\mathrm{loc}} / \mathscr{I}_{v}^{\left(\chi_{v, 1}, \ldots, \chi_{v, n}\right)}=\mathscr{O}_{\mathscr{M}}^{\wedge}\left(\prod_{i=1}^{n}\left(\mathrm{X}-\chi_{v, i}\left(\sigma_{\widetilde{v}}\right)\right), \# k(v)\right),\left(1_{n}, 1_{n}\right) \cdot
$$

Let $\wp$ be a maximal ideal of $\mathrm{R}[1 / l]$. The residue field $k(\wp)$ is a finite extension of K. Let $\mathscr{O}_{k(\wp)}^{\prime}$ denote the subring of $\mathscr{O}_{k(\wp)}$ consisting of elements which reduce modulo the maximal ideal to an element of $k$. Then $\mathrm{R} / \wp^{c}$ is a subring of $\mathscr{O}_{k(\wp)}^{\prime}$. (See Lemma 2.6.)

also that

Suppose that $\mathrm{A}$ is a $\mathrm{K}$-algebra with an ideal $\mathrm{I}$ satisfying $\mathrm{I}^{2}=(0)$. Suppose

$$
f: \mathrm{R}[1 / l] / \wp^{r} \longrightarrow \mathrm{A} / \mathrm{I}
$$

is a map of $\mathrm{K}$-algebras. We must show that $f$ can be lifted to a map

$$
\tilde{f}: \mathrm{R}[1 / l] / \wp^{2 r} \longrightarrow \mathrm{A} .
$$

First of all we may replace $\mathrm{A}$ by the preimage in $\mathrm{A}$ of the image of $f$ in $\mathrm{A} / \mathrm{I}$. Thus we may suppose that $\mathrm{A}$ is local with nilpotent maximal ideal. Secondly we may replace A by a finitely generated K-subalgebra, so we may suppose that A is in fact an Artinian local ring. In particular the homomorphism $\mathrm{A} \rightarrow k(\wp)$ has a section (because A contains the field $\mathrm{K}$ ) and we can consider $\mathrm{A}$ as a $k(\wp)$ algebra. (For instance, by Theorem 7.7 of [E].)

Let $(\Phi, \Sigma) \in \mathrm{GL}_{n}(\mathrm{~A} / \mathrm{I})^{2}$ denote the image of the universal matrices over $\mathscr{M}\left(\prod_{i=1}^{n}\left(\mathrm{X}-\chi_{v, i}\left(\sigma_{\widetilde{v}}\right)\right), \# k(v)\right)$. As the roots of the characteristic polynomial of $\Sigma$ are distinct elements of $\mathrm{K}$ we may find a basis $e_{1}, \ldots, e_{n}$ of $(\mathrm{A} / \mathrm{I})^{n}$ so that

$$
\sum e_{i}=\chi_{v, i}\left(\sigma_{\widetilde{v}}\right) e_{i}
$$

As $\Sigma^{\# k(v)}=\Sigma$, we must have $\Sigma \Phi=\Phi \Sigma$ and so

$$
\Phi e_{i}=\alpha_{i} e_{i}
$$

for some $\alpha_{i} \in \mathrm{A} / \mathrm{I}$. Choose a lifting of $\left\{e_{1}, \ldots, e_{n}\right\}$ to a basis $\left\{\widetilde{e}_{1}, \ldots, \widetilde{e}_{n}\right\}$ of $\mathrm{A}^{n}$ and a lifting $\widetilde{\alpha}_{i}$ to A of each $\alpha_{i}$. Define a lifting $(\widetilde{\Phi}, \widetilde{\Sigma})$ of $(\Phi, \Sigma)$ to A by

$$
\widetilde{\Sigma} \widetilde{e_{i}}=\chi_{v, i}\left(\sigma_{\widetilde{v}}\right) \widetilde{e_{i}}
$$


and

$$
\widetilde{\Phi} \widetilde{e_{i}}=\widetilde{\alpha}_{i}{\widetilde{e_{i}}}_{\text {. }}
$$

Then $\widetilde{\Sigma}$ has characteristic polynomial

$$
\prod_{i=1}^{n}\left(\mathrm{X}-\chi_{v, i}\left(\sigma_{\widetilde{v}}\right)\right)
$$

and

$$
\widetilde{\Phi} \widetilde{\Sigma} \widetilde{\Phi}^{-1}=\widetilde{\Sigma}^{\# k(v)}
$$

Note that the reductions $\bar{\Phi}$ and $\bar{\Sigma}$ of $\Phi$ and $\Sigma$ modulo the maximal ideal of A lie in $\mathscr{O}_{k(\wp)}^{\prime}$. The entries of $\widetilde{\Phi}-\bar{\Phi}$ and $\widetilde{\Sigma}-\bar{\Sigma}$ are in the maximal ideal of $\mathrm{A}$ and hence nilpotent. Let $\mathrm{A}^{0}$ denote the $\mathscr{O}_{k(\wp)}^{\prime}$-subalgebra of $\mathrm{A}$ generated by the entries of $\widetilde{\Phi}-\bar{\Phi}$ and $\widetilde{\Sigma}-\bar{\Sigma}$. Then $\mathrm{A}^{0}$ is a complete noetherian local $\mathscr{O}$-algebra with residue field $k$. Thus we can find a map of $\mathscr{O}$-algebras

$$
\mathrm{R} \longrightarrow \mathrm{A}^{0}
$$

so that the universal matrices over $\mathscr{M}\left(\prod_{i=1}^{n}\left(\mathrm{X}-\chi_{v, i}\left(\sigma_{\widetilde{v}}\right)\right), \# k(v)\right)$ map to $(\widetilde{\Phi}, \widetilde{\Sigma})$. This map extends to the desired map

$$
\tilde{f}: \mathrm{R}[1 / l] \longrightarrow \mathrm{A} \text {. }
$$

The proof in the case

$$
\mathrm{R}=\mathrm{R}_{v}^{\text {loc }} / \mathscr{I}_{v}^{\text {Stein }}=\mathscr{O}_{\mathscr{M}^{\text {Stein }}(\# k(v)),\left(1_{n}, 1_{n}\right)}^{\wedge}
$$

is very similar. The key point is to show that $(\Phi, \Sigma) \in \mathrm{GL}_{n}(\mathrm{~A} / \mathrm{I})^{2}$ lifts to $(\widetilde{\Phi}, \widetilde{\Sigma}) \in$ $\mathrm{GL}_{n}(\mathrm{~A})^{2}$ such that $\widetilde{\Sigma}$ has characteristic polynomial $(\mathrm{X}-1)^{n}$, while $\widetilde{\Phi}$ has characteristic polynomial in $\operatorname{Pol}_{n}(\{n\}, \# k(v))$ and $\widetilde{\Phi} \widetilde{\Sigma} \widetilde{\Phi}^{-1}=\widetilde{\Sigma}^{\# k(v)}$. The characteristic polynomial

$$
\operatorname{char}_{\Phi}(\mathrm{X})=\prod_{i=1}^{n}\left(\mathrm{X}-\alpha q^{i-1}\right)
$$

where

$$
\alpha=(\operatorname{tr} \Phi) /\left(1+q+\ldots+q^{n-1}\right) .
$$

As the roots of $\operatorname{char}_{\Phi}(\mathrm{X})$ are distinct modulo the maximal ideal of $\mathrm{A} / \mathrm{I}$, we can choose a basis $e_{1}, \ldots, e_{n}$ of $(\mathrm{A} / \mathrm{I})^{n}$ such that

$$
\Phi e_{i}=\alpha q^{i-1} e_{i}
$$


Then we must have

$$
\sum e_{i}=\beta_{i} e_{i-1}
$$

where $\beta_{i} \in \mathrm{A} / \mathrm{I}$ and where we set $e_{0}=0$. Choose a lifting of $\left\{e_{1}, \ldots, e_{n}\right\}$ to a basis $\left\{\widetilde{e}_{1}, \ldots, \widetilde{e}_{n}\right\}$ of $\mathrm{A}^{n}$, a lifting $\widetilde{\alpha}$ of $\alpha$ to $\mathrm{A}$ and a lifting $\widetilde{\beta}_{i}$ of each $\beta_{i}$ to A. Define liftings $(\widetilde{\Phi}, \widetilde{\Sigma})$ of $(\Phi, \Sigma)$ to A by

$$
\widetilde{\Phi} \widetilde{e_{i}}=\widetilde{\alpha} q^{i-1} \widetilde{e}_{i}
$$

and

$$
\widetilde{\Sigma} e_{i}=\widetilde{\beta}_{i} \widetilde{e}_{i-1}
$$

(with $\tilde{e}_{0}=0$ ). This lifting has the desired properties, and allows one to complete the proof of the lemma in the second case.

nected.

Lemma 3.4. - $\operatorname{Spec}\left(\mathrm{R}_{v}^{\mathrm{loc}} / \mathscr{I}_{v}^{\left(\chi_{v, 1}, \ldots, \chi_{v, n}\right)}\right)[1 / l]$ and $\operatorname{Spec}\left(\mathrm{R}_{v}^{\text {loc }} / \mathscr{I}_{v}^{\text {Stein }}\right)[1 / l]$ are con-

Proof. - Consider first the case of

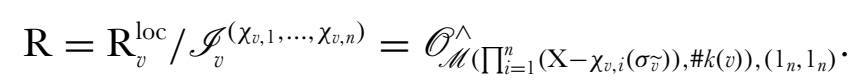

Let $\wp_{0}$ be the maximal ideal of $\mathrm{R}[1 / l]$ corresponding to

$$
\left(\Phi_{0}, \Sigma_{0}\right)=\left(1_{n}, \operatorname{diag}\left(\chi_{v, n}\left(\sigma_{\widetilde{v}}\right), \ldots, \chi_{v, 1}\left(\sigma_{\widetilde{v}}\right)\right)\right) \in \mathrm{M}_{n}(\mathrm{~K})^{2} .
$$

We will show that any other maximal ideal $\wp$ of $\mathrm{R}[1 / l]$ is in the same connected component. The result will then follow from Lemma 2.6.

The residue field $k(\wp)$ is a finite extension of $\mathrm{K}$. Let $\mathscr{O}_{k(\wp)}$ denote its ring of integers and and $\mathfrak{m}_{k(\wp)}$ the maximal ideal of $\mathscr{O}_{k(\wp)}$. Then $\mathrm{R} / \wp^{c} \hookrightarrow \mathscr{O}_{k(\wp)}$, and this corresponds to some $(\Phi, \Sigma) \in \mathrm{M}_{n}\left(\mathscr{O}_{k(\wp)}\right)^{2}$. (See Lemma 2.6.) Let A denote the complete topological domain

$$
\mathscr{O}_{k(\wp)}\left\langle\mathrm{X}_{i j}, \mathrm{Y}\right\rangle_{i, j=1, \ldots, n} /\left(\mathrm{Y} \operatorname{det}\left(\mathrm{X}_{i j}\right)-1\right)
$$

where \langle\rangle denotes power series whose coefficients tend to zero. (The topology being the $\mathfrak{m}_{k(\wp)}$ topology.) Consider the pair

$$
\left(\left(\mathrm{X}_{i j}\right)^{-1} \Phi\left(\mathrm{X}_{i j}\right),\left(\mathrm{X}_{i j}\right)^{-1} \Sigma\left(\mathrm{X}_{i j}\right)\right) .
$$

This defines a map

$$
\operatorname{Spec} \mathrm{A} \longrightarrow \mathscr{M}\left(\prod_{i=1}^{n}\left(\mathrm{X}-\chi_{v, i}\left(\sigma_{\widetilde{v}}\right)\right), \# k(v)\right)
$$


such that $\operatorname{Spec} \mathrm{A} / \mathfrak{m}_{k(\wp)} \mathrm{A}$ maps to the point

$$
\left(1_{n}, 1_{n}\right) \in \mathscr{M}\left(\prod_{i=1}^{n}\left(\mathrm{X}-\chi_{v, i}\left(\sigma_{\widetilde{v}}\right)\right), \# k(v)\right)(k) .
$$

Thus we get a (continuous) homomorphism

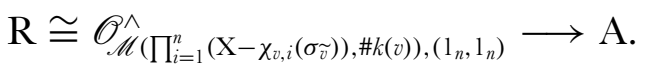

We conclude that $\wp$ is in the same connected component of Spec $R[1 / l]$ as the maximal ideal corresponding to $\left(\mathrm{E}^{-1} \Phi \mathrm{E}, \mathrm{E}^{-1} \Sigma \mathrm{E}\right)$ for any $\mathrm{E} \in \mathrm{GL}_{n}\left(\mathscr{O}_{k(\wp)}\right)$.

We can choose a decreasing filtration $\mathrm{Fil}^{i}$ of $k(\wp)^{n}$ such that

- each $\mathrm{Fil}^{i}$ is a preserved by $\Phi$ and $\Sigma$ and

- for $i=1, \ldots, n$ the graded piece $\operatorname{gr}^{i}$ is one dimensional and $\Sigma$ acts on it by $\chi_{v, i}\left(\sigma_{\widetilde{v}}\right)$.

(As $\chi_{v, i}\left(\sigma_{\widetilde{v}}\right)^{\# k(\widetilde{v})}=\chi_{v, i}\left(\sigma_{\widetilde{v}}\right)$ we see that $\Phi$ preserves $\operatorname{ker}\left(\Sigma-\chi_{v, i}\left(\sigma_{\widetilde{v}}\right)\right)^{a}$ for all positive integers a.) Let $e_{1}, \ldots, e_{n}$ be a basis of $\mathscr{O}_{k(\wp)}^{n}$ such that $e_{i}, \ldots, e_{n}$ is a $k(\wp)$-basis of Fil $^{i}$ for all $i=1, \ldots, n$, and let

$$
\mathrm{E}=\left(e_{n} e_{n-1} \ldots e_{1}\right) \in \mathrm{GL}_{n}(\mathscr{O}) \text {. }
$$

Then $\mathrm{E}^{-1} \Phi \mathrm{E} \in \mathrm{GL}_{n}\left(\mathscr{O}_{k(\wp)}\right)$ is upper triangular and $\mathrm{E}^{-1} \Sigma \mathrm{E} \in \mathrm{GL}_{n}\left(\mathscr{O}_{k(\wp)}\right)$ is upper triangular with diagonal entries $\chi_{v, n}\left(\sigma_{\widetilde{v}}\right), \ldots, \chi_{v, 1}\left(\sigma_{\widetilde{v}}\right)$ (reading from top left to bottom right).

Thus we are reduced to the case that $\Phi$ is upper triangular and $\Sigma$ is upper triangular with diagonal entries $\chi_{v, n}\left(\sigma_{\widetilde{v}}\right), \ldots, \chi_{v, 1}\left(\sigma_{\widetilde{v}}\right)$ (reading from top left to bottom right). Let

$$
\mathrm{B}=\mathscr{O}_{k(\wp)}\langle\mathrm{X}\rangle .
$$

Let $\Lambda=\operatorname{diag}\left(1, \mathrm{X}, \ldots, \mathrm{X}^{n-1}\right)$ and consider the pair

$$
\left(\Lambda^{-1} \Phi \Lambda, \Lambda^{-1} \Sigma \Lambda\right) \text {. }
$$

This defines a map

$$
\operatorname{Spec} \mathrm{B} \longrightarrow \mathscr{M}\left(\prod_{i=1}^{n}\left(\mathrm{X}-\chi_{v, i}\left(\sigma_{\widetilde{v}}\right)\right), \# k(v)\right)
$$

such that $\operatorname{Spec} B / \mathfrak{m}_{k(w)}$ maps to the point

$$
\left(1_{n}, 1_{n}\right) \in \mathscr{M}\left(\prod_{i=1}^{n}\left(\mathrm{X}-\chi_{v, i}\left(\sigma_{\widetilde{v}}\right)\right), \# k(v)\right)(k) .
$$


(Although $\Lambda^{-1}$ is not defined over $\mathrm{B}$, the products $\Lambda^{-1} \Phi \Lambda$ and $\Lambda^{-1} \Sigma \Lambda$ are defined over B.) Thus we get a (continuous) homomorphism

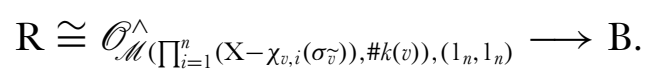

The point of $\mathrm{R}[1 / l]$ corresponding to the map $\mathrm{B} \rightarrow \mathscr{O}_{k(\wp)}$ sending $\mathrm{X}$ to 1 is $\wp$. The point of $\mathrm{R}[1 / l]$ corresponding to the map $\mathrm{B} \rightarrow \mathscr{O}_{k(\wp)}$ sending $\mathrm{X}$ to 0 corresponds to a pair

$$
\left(\operatorname{diag}\left(\alpha_{1}, \ldots, \alpha_{n}\right), \operatorname{diag}\left(\chi_{v, n}\left(\sigma_{\widetilde{v}}\right), \ldots, \chi_{v, 1}\left(\sigma_{\widetilde{v}}\right)\right)\right) .
$$

These two points are in the same connected component of Spec R[1/l].

Thus we are reduced to the case that $\Sigma=\operatorname{diag}\left(\chi_{v, n}\left(\sigma_{\widetilde{v}}\right), \ldots, \chi_{v, 1}\left(\sigma_{\widetilde{v}}\right)\right)$ and $\Phi=\operatorname{diag}\left(\alpha_{1}, \ldots, \alpha_{n}\right)$. Let

$$
\mathrm{C}=\mathscr{O}_{k(\wp)}\left[\left[\mathrm{X}_{1}, \ldots, \mathrm{X}_{n}\right]\right]
$$

Consider the pair

$$
\left(\operatorname{diag}\left(1+\mathrm{X}_{1}, \ldots, 1+\mathrm{X}_{n}\right)^{-1} \Phi, \Sigma\right) .
$$

This defines a map

$$
\operatorname{Spec} \mathrm{C} \longrightarrow \mathscr{M}\left(\prod_{i=1}^{n}\left(\mathrm{X}-\chi_{v, i}\left(\sigma_{\widetilde{v}}\right)\right), \# k(v)\right)
$$

such that Spec $\mathrm{C} /\left(\mathrm{X}_{1}, \ldots, \mathrm{X}_{n}, \lambda\right)$ maps to the point

$$
\left(1_{n}, 1_{n}\right) \in \mathscr{M}\left(\prod_{i=1}^{n}\left(\mathrm{X}-\chi_{v, i}\left(\sigma_{\widetilde{v}}\right)\right), \# k(v)\right)(k) .
$$

Thus we get a (continuous) homomorphism

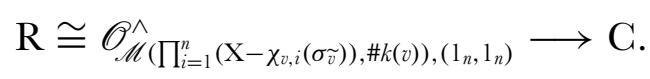

The point $\wp$ corresponds to the map $\mathrm{C} \rightarrow \mathscr{O}_{k(\wp)}$ sending $\mathrm{X}_{i} \mapsto 0$ for all $i$; while the point $\wp_{0}$ corresponds to the map $\mathrm{C} \rightarrow \mathscr{O}_{k(\wp)}$ sending $\mathrm{X}_{i} \mapsto \alpha_{i}-1$ for all $i$. Thus $\wp$ and $\wp_{0}$ are in the same connected component of Spec $R[1 / l]$, and the lemma follows.

Consider now the case

$$
\mathrm{R}=\mathrm{R}_{v}^{\mathrm{loc}} / \mathscr{I}_{v}^{\text {Stein }}=\mathscr{O}_{\mathscr{M}^{\text {Stein }}(\# k(v)),\left(1_{n}, 1_{n}\right)}^{\wedge} .
$$


Let $\wp_{0}$ be the maximal ideal of $\mathrm{R}[1 / l]$ corresponding to

$$
(\Phi, \Sigma)=\left(\operatorname{diag}\left((\# k(v))^{n-1}, \ldots,(\# k(v)), 1\right), 1_{n}\right) \in \mathrm{M}_{n}(\mathrm{~K})^{2} .
$$

We will show that any other maximal ideal $\wp$ of $\mathrm{R}[1 / l]$ is in the same connected component. The argument is much the same as the previous case, so we will simply sketch it. We first reduce to the case that $\Sigma$ is upper triangular with l's on the diagonal and $\Phi$ is upper triangular with entries $\alpha(\# k(v))^{n-1}, \ldots, \alpha(\# k(v)), \alpha$ (reading from top left to bottom right). Then one reduces to the case that $\Sigma$ and $\Phi$ are diagonal of the same form and finally to the case $\alpha=1$.

Finally we complete the proof of Proposition 3.1.

Proof of Proposition 3.1. - Consider the first part of the proposition. Lemma 3.3 tells us that for any maximal ideal $\wp$ of $\left(\mathrm{R}_{v}^{\operatorname{loc}} / \mathscr{I}_{v}\left(\chi_{v, 1}, \ldots, \chi_{v, n}\right)[1 / l]\right.$ the localisation $\left(\mathrm{R}_{v}^{\mathrm{loc}} / \mathscr{I}_{v}^{(}\left(\chi_{v, 1}, \ldots, \chi_{v, n}\right)\right)[1 / l]_{\wp}$ is a domain. Combining this with Lemma 3.4 and the fact that the maximal ideals are dense in the spectrum $\operatorname{Spec}\left(\mathrm{R}_{v}^{\text {loc }} / \mathscr{I}_{v}^{\left(\chi_{v, 1}, \ldots, \chi_{v, n}\right)}\right)[1 / l]$, we see that $\left(\mathrm{R}_{v}^{\text {loc }} / \mathscr{I}_{v}^{\left(\chi_{v, 1}, \ldots, \chi_{v, n}\right)}\right)[1 / l]$ is an integral domain. Lemma 3.2 implies that $\left(\mathrm{R}_{v}^{\text {loc }} / \mathscr{I}_{v}\left(\chi_{v, 1}, \ldots, \chi_{v, n}\right)\right)^{\text {red }}$ is flat over $\mathscr{O}$ and we deduce that this ring is also an integral domain.

The second part of the proposition follows from the definitions. The third part follows from Lemmas 2.7 and 3.2. The fourth part follows from Lemmas 3.3 and 3.4 in the same way that the first part did. (Except in this case $\mathrm{R}_{v}^{\text {loc }} / \mathscr{J}_{v}^{\text {Stein }}$ is flat over $\mathscr{O}$ by definition.)

\section{An $\mathrm{R}^{\text {red }}=\mathbf{T}$ theorem}

Fix a positive integer $n \geq 2$ and a prime $l>n$.

Fix an imaginary quadratic field $\mathrm{E}$ in which $l$ splits and a totally real field $\mathrm{F}^{+}$such that

$-\mathrm{F}=\mathrm{F}^{+} \mathrm{E} / \mathrm{F}^{+}$is unramified at all finite primes, and

$-\mathrm{F}^{+} / \mathbf{Q}$ is unramified at $l$.

Fix a finite non-empty set of places $\mathrm{S}(\mathrm{B})$ of places of $\mathrm{F}^{+}$with the following properties:

- Every element of $\mathrm{S}(\mathrm{B})$ splits in $\mathrm{F}$.

- S(B) contains no place above $l$.

- If $n$ is even then

$$
n\left[\mathrm{~F}^{+}: \mathbf{Q}\right] / 2+\# \mathrm{~S}(\mathrm{~B}) \equiv 0 \bmod 2 .
$$

Choose a division algebra $\mathrm{B}$ with centre $\mathrm{F}$ with the following properties: 
$-\operatorname{dim}_{\mathrm{F}} \mathrm{B}=n^{2}$.

$-\mathrm{B}^{\mathrm{op}} \cong \mathrm{B} \otimes_{\mathrm{E}, c} \mathrm{E}$.

- B splits outside $\mathrm{S}(\mathrm{B})$.

- If $w$ is a prime of $\mathrm{F}$ above an element of $\mathrm{S}(\mathrm{B})$, then $\mathrm{B}_{w}$ is a division algebra.

Also choose an involution $\ddagger$ on $\mathrm{B}$ and define an algebraic group $\mathrm{G} / \mathrm{F}^{+}$by

$$
\mathrm{G}(\mathrm{A})=\left\{g \in \mathrm{B} \otimes_{\mathrm{F}^{+}} \mathrm{A}: g^{\ddagger \otimes 1} g=1\right\},
$$

such that

$-\left.\ddagger\right|_{\mathrm{F}}=c$,

- for a place $v \mid \infty$ of $\mathrm{F}^{+}$we have $\mathrm{G}\left(\mathrm{F}_{v}^{+}\right) \cong \mathrm{U}(n)$, and

- for every finite place $v \notin \mathrm{S}(\mathrm{B})$ of $\mathrm{F}^{+}$the group $\mathrm{G}\left(\mathrm{F}_{v}^{+}\right)$is quasi-split.

Because of the first and third itemized assumptions on $\mathrm{S}(\mathrm{B})$, it is always possible to choose such a B and then such a $\ddagger$. (The argument is exactly analogous to the proof of Lemma 1.7.1 of [HT].) The purpose of the assumption that $\mathrm{S}(\mathrm{B}) \neq \varnothing$ is to simplify the use of the trace formula in relating automorphic forms on $G$ to automorphic forms on $\mathrm{GL}_{n} / \mathrm{F}$ and in attaching Galois representations to automorphic forms on $\mathrm{G}$.

Choose an order $\mathscr{O}_{\mathrm{B}}$ in $\mathrm{B}$ such that $\mathscr{O}_{\mathrm{B}}^{\ddagger}=\mathscr{O}_{\mathrm{B}}$ and $\mathscr{O}_{\mathrm{B}, w}$ is maximal for all primes $w$ of $\mathrm{F}$ which are split over $\mathrm{F}^{+}$. (See Section 3.3 of [CHT].) This gives a model of $\mathrm{G}$ over $\mathscr{O}_{\mathrm{F}^{+}}$. If $v \notin \mathrm{S}(\mathrm{B})$ is a prime of $\mathrm{F}^{+}$which splits in $\mathrm{F}$ choose an isomorphism $i_{v}: \mathscr{O}_{\mathrm{B}, v} \stackrel{\sim}{\rightarrow} \mathrm{M}_{n}\left(\mathscr{O}_{\mathrm{F}_{v}}\right)$ such that $i_{v}\left(x^{\ddagger}\right)={ }^{t} i_{v}(x)^{c}$. The choice of a prime $w$ of $\mathrm{F}$ above $v$ then gives us an identification

$$
\begin{aligned}
& i_{w}: \mathrm{G}\left(\mathrm{F}_{v}^{+}\right) \stackrel{\sim}{\longrightarrow} \mathrm{GL}_{n}\left(\mathrm{~F}_{w}\right) \\
& i_{v}^{-1}\left(x,{ }^{t} x^{-c}\right) \longmapsto x
\end{aligned}
$$

with $i_{w} \mathrm{G}\left(\mathscr{O}_{\mathrm{F}^{+}, v}\right)=\mathrm{GL}_{n}\left(\mathscr{O}_{\mathrm{F}, w}\right)$ and $i_{w^{c}}={ }^{t}\left(c \circ i_{w}\right)^{-1}$. If $v \in \mathrm{S}(\mathrm{B})$ and $w$ is a prime of $\mathrm{F}$ above $v$ choose isomorphisms $i_{w}: \mathrm{G}\left(\mathrm{F}_{v}^{+}\right) \stackrel{\sim}{\rightarrow} \mathrm{B}_{w}^{\times}$such that $i_{w}=i_{w}^{-\ddagger}$ and $i_{w} \mathrm{G}\left(\mathscr{O}_{\mathrm{F}^{+}, v}\right)=\mathscr{O}_{\mathrm{B}, w}^{\times}$.

If $w$ is a finite place of $\mathrm{F}$ which is split over a place $v \notin \mathrm{S}(\mathrm{B})$ of $\mathrm{F}^{+}$, then write $\operatorname{Iw}(w)$ for the inverse image under $i_{w}$ of the group of matrices in $\operatorname{GL}_{n}\left(\mathscr{O}_{\mathrm{F}, w}\right)$ which reduce modulo $w$ to an upper triangular matrix. There is a natural homomorphism

$$
\operatorname{Iw}(w) \rightarrow\left(k(w)^{\times}\right)^{n},
$$

which takes $g$ to the diagonal entries of the reduction modulo $w$ of $i_{w}(g)$. Denote the kernel of this map by $\operatorname{Iw}_{1}(w)$. Similarly write $\mathrm{U}_{0}(w)$ (resp. $\mathrm{U}_{1}(w)$ ) for the inverse image under $i_{w}$ of the group of matrices in $\mathrm{GL}_{n}\left(\mathscr{O}_{\mathrm{F}, w}\right)$ whose reduction 
modulo $w$ has last row $(0, \ldots, 0, *)$ (resp. $(0, \ldots, 0,1))$. Then $\mathrm{U}_{1}(w)$ is a normal subgroup of $\mathrm{U}_{0}(w)$ and we have a natural isomorphism

$$
\mathrm{U}_{0}(w) / \mathrm{U}_{1}(w) \stackrel{\sim}{\longrightarrow} k(w)^{\times} .
$$

(It sends $g$ to the lower right entry of the reduction modulo $w$ of $i_{w}(g)$.)

Let $\mathrm{S}_{l}$ denote the set of primes of $\mathrm{F}^{+}$above $l$. Let $\mathrm{S}_{a}$ denote a non-empty set, disjoint from $\mathrm{S}_{l} \cup \mathrm{S}(\mathrm{B})$, of primes of $\mathrm{F}^{+}$such that

- if $v \in \mathrm{S}_{a}$ then $v$ splits in $\mathrm{F}$, and

- if $v \in \mathrm{S}_{a}$ lies above a rational prime $p$ then $\left[\mathrm{F}\left(\zeta_{p}\right): \mathrm{F}\right]>n$.

(The only role played by the primes in $\mathrm{S}_{a}$ will be to ensure that certain open compact subgroups of $\mathrm{G}\left(\mathbf{A}_{\mathrm{F}^{+}}^{\infty}\right)$ are sufficiently small.) Let $\mathrm{R}$ denote a set, disjoint from $\mathrm{S}_{l} \cup \mathrm{S}(\mathrm{B}) \cup \mathrm{S}_{a}$, of primes of $\mathrm{F}^{+}$such that

- if $v \in \mathbf{R}$ then $v$ splits in $\mathbf{F}$, and

- if $v \in \mathbf{R}$ then $\mathbf{N} v \equiv 1 \bmod l$.

(The set $\mathrm{R}$ is to allow us to prove modularity of $l$-adic representations ramified at more primes than their $\bmod l$ reduction. These extra primes of ramification will be the elements of R.) Fix a decomposition

$$
\mathrm{S}(\mathrm{B})=\mathrm{S}(\mathrm{B})_{1} \coprod \mathrm{S}(\mathrm{B})_{2} \text {. }
$$

(This will correspond to two different sorts of discrete series deformations we will allow.) Let $\mathrm{T}=\mathrm{R} \cup \mathrm{S}(\mathrm{B}) \cup \mathrm{S}_{l} \cup \mathrm{S}_{a}$. Let $\widetilde{\mathrm{T}}$ denote a set of primes of $\mathrm{F}$ above $\mathrm{T}$ such that $\widetilde{\mathrm{T}} \coprod \widetilde{\mathrm{T}}^{c}$ is the set of all primes of $\mathrm{F}$ above $\mathrm{T}$. If $v \in \mathrm{T}$ we will let $\widetilde{v}$ denote the prime of $\widetilde{\mathrm{T}}$ above $v$. If $\mathrm{S} \subset \mathrm{T}$ we will let $\widetilde{\mathrm{S}}$ denote the set of $\widetilde{v}$ for $v \in \mathrm{S}$.

Let $\mathrm{U}=\prod_{v} \mathrm{U}_{v}$ denote an open compact subgroup of $\mathrm{G}\left(\mathbf{A}_{\mathrm{F}^{+}}^{\infty}\right)$ such that

- if $v$ is not split in $\mathrm{F}$ then $\mathrm{U}_{v}$ is a hyperspecial maximal compact subgroup of $\mathrm{G}\left(\mathrm{F}_{v}^{+}\right)$,

- if $v \notin \mathrm{S}_{a} \cup \mathrm{R}$ splits in $\mathrm{F}$ then $\mathrm{U}_{v}=\mathrm{G}\left(\mathscr{O}_{\mathrm{F}^{+}, v}\right)$,

- if $v \in \mathrm{R}$ then $\mathrm{U}_{v}=\operatorname{Iw}(\widetilde{v})$, and

- if $v \in \mathrm{S}_{a}$ then $\mathrm{U}_{v}=i_{\widetilde{v}}^{-1} \operatorname{ker}\left(\mathrm{GL}_{n}\left(\mathscr{O}_{\mathrm{F}, \widetilde{v}}\right) \rightarrow \mathrm{GL}_{n}(k(\widetilde{v}))\right)$.

Then $U$ is sufficiently small in the sense that for some place $v$ its projection to $\mathrm{G}\left(\mathrm{F}_{v}^{+}\right)$contains only one element of finite order, namely 1. (In fact for $v \in \mathrm{S}_{a}$.)

If $\mathrm{Q}$ is a finite set of primes of $\mathrm{F}^{+}$which split in $\mathrm{F}$ and if $\mathrm{Q}$ is disjoint from $\mathrm{T}$, then we will write $\mathrm{T}(\mathrm{Q})$ for $\mathrm{T} \cup \mathrm{Q}$ and $\widetilde{\mathrm{T}}(\mathrm{Q}) \supset \widetilde{\mathrm{T}}$ for a set consisting of one place of $\mathrm{F}$ above each place in $\mathrm{T}(\mathrm{Q})$. We will also write $\mathrm{U}_{0}(\mathrm{Q})$ for $\prod_{v \notin \mathrm{Q}} \mathrm{U}_{v} \times \prod_{v \in \mathrm{Q}} \mathrm{U}_{0}(\tilde{v})$, and $\mathrm{U}_{1}(\mathrm{Q})$ for $\prod_{v \notin \mathrm{Q}} \mathrm{U}_{v} \times \prod_{v \in \mathrm{Q}} \mathrm{U}_{1}(\tilde{v})$. 
Let $\mathrm{K} / \mathbf{Q}_{l}$ be a finite extension which contains a primitive $l^{\text {th }}$ root of unity and contains the image of every embedding $\mathrm{F}^{+} \hookrightarrow \overline{\mathrm{K}}$. Let $\mathscr{O}$ denote its ring of integers, $\lambda$ the maximal ideal of $\mathscr{O}$ and $k$ the residue field $\mathscr{O} / \lambda$.

For $v \in \mathrm{R}$ let

$$
\chi_{v}=\chi_{v, 1} \times \ldots \times \chi_{v, n}: \operatorname{Iw}(\widetilde{v}) / \operatorname{Iw}_{1}(\widetilde{v}) \longrightarrow \mathscr{O}^{\times}
$$

denote a character of order dividing $l$. We will be particularly interested in the case $\chi_{v}=1$ and the case $\chi_{v, i} \neq \chi_{v, j}$ for all $i \neq j$. (Recall that $l>n$.)

For each $\tau: \mathrm{F} \hookrightarrow \mathrm{K}$ choose integers $a_{\tau, 1}, \ldots, a_{\tau, n}$ such that

$-a_{\tau c, i}=-a_{\tau, n+1-i}$, and

- if $\tau$ gives rise to a place in $\widetilde{\mathrm{S}}_{l}$ then

$$
l-1-n \geq a_{\tau, 1} \geq \ldots \geq a_{\tau, n} \geq 0 .
$$

We get a K-vector space $\mathrm{W}_{a}$ and an irreducible representation

$$
\begin{aligned}
\xi_{a}: \mathrm{G}\left(\mathrm{F}_{l}^{+}\right) & \longrightarrow \mathrm{GL}\left(\mathrm{W}_{a}\right) \\
g & \longmapsto \bigotimes_{\tau \in \widetilde{\mathrm{I}}_{l}} \xi_{a_{\tau}}\left(\tau i_{\tau} g\right)
\end{aligned}
$$

coming from the algebraic representation with highest weight $a$. (See Section 3.3 of $[\mathrm{CHT}]$.$) The representation \xi_{a}$ contains a $\mathrm{G}\left(\mathscr{O}_{\mathrm{F}^{+}, l}\right)$-invariant $\mathscr{O}$-lattice $\mathrm{M}_{a}$.

For each $v \in \mathrm{S}(\mathrm{B})$ let $\rho_{v}: \mathrm{G}\left(\mathrm{F}_{v}^{+}\right) \longrightarrow \mathrm{GL}\left(\mathrm{M}_{\rho_{v}}\right)$ denote a representation of $\mathrm{G}\left(\mathrm{F}_{v}^{+}\right)$on a finite free $\mathscr{O}$-module such that $\rho_{v}$ has open kernel and $\mathrm{M}_{\rho_{v}} \otimes_{\mathscr{O}} \overline{\mathrm{K}}$ is irreducible. For $v \in \mathrm{S}(\mathrm{B})$, define $m_{v}, \pi_{\widetilde{v}}$ and $\tilde{r}_{\widetilde{v}}$ by

$$
\operatorname{JL}\left(\rho_{v} \circ i_{\widetilde{v}}^{-1}\right)=\operatorname{Sp}_{m_{v}}\left(\pi_{\widetilde{v}}\right)
$$

and

$$
\tilde{r}_{\widetilde{v}}=r_{l}\left(\pi_{\widetilde{v}}||^{\left(n / m_{v}-1\right)\left(1-m_{v}\right) / 2}\right) .
$$

(Here we let JL denote the Jacquet-Langlands map and $\operatorname{Sp}_{m}(\sigma)$ a generalised Steinberg representation as in Section I.3 of [HT]. We also let $r_{l}$ denote the reciprocity map with its 'arithmetic normalisation' as in Section VII.1 of [HT].) We will suppose that

$$
\widetilde{r}_{\widetilde{v}}: \operatorname{Gal}\left(\overline{\mathrm{F}}_{\widetilde{v}} / \mathrm{F}_{\widetilde{v}}\right) \longrightarrow \mathrm{GL}_{n / m_{v}}(\mathscr{O})
$$

(as opposed to $\mathrm{GL}_{n / m_{v}}(\overline{\mathrm{K}})$ ). If $v \in \mathrm{S}(\mathrm{B})_{1}$ we will further suppose that $\mathbf{N} v \equiv 1 \bmod l$, that $m_{v}=n$ and that $\pi_{\widetilde{v}}$ is unramified. If $v \in \mathrm{S}(\mathrm{B})_{2}$ we will further suppose that the reduction of $\tilde{r}_{\widetilde{v}} \bmod \lambda$ is absolutely irreducible and

$$
\tilde{r}_{\widetilde{v}} \otimes_{\mathscr{O}} k \supsetneqq \tilde{r}_{\widetilde{v}} \otimes_{\mathscr{O}} k\left(\epsilon^{j}\right)
$$


for $j=1, \ldots, m_{v}$. (It should be possible to treat the common generalisation of these two extreme cases, but we will not do so here.)

Set

$$
\mathrm{M}_{a,\left\{\rho_{v}\right\},\left\{\chi_{v}\right\}}=\mathrm{M}_{a} \otimes \bigotimes_{v \in \mathrm{S}(\mathrm{B})} \mathrm{M}_{\rho_{v}} \otimes \bigotimes_{v \in \mathrm{R}} \mathscr{O}\left(\chi_{v}\right)
$$

Suppose that either $\mathrm{A}$ is a $\mathrm{K}$-algebra or that the projection of $\mathrm{U}$ to $\mathrm{G}\left(\mathrm{F}_{l}^{+}\right)$is contained in $\mathrm{G}\left(\mathscr{O}_{\mathrm{F}^{+}, l}\right)$. Then we define a space of automorphic forms

$$
\mathrm{S}_{a,\left\{\rho_{v}\right\},\left\{\chi_{v}\right\}}(\mathrm{U}, \mathrm{A})
$$

to be the space of functions

$$
f: \mathrm{G}\left(\mathrm{F}^{+}\right) \backslash \mathrm{G}\left(\mathbf{A}_{\mathrm{F}^{+}}^{\infty}\right) \longrightarrow \mathrm{A} \otimes_{\mathscr{O}} \mathrm{M}_{a,\left\{\rho_{v}\right\},\left\{\chi_{v}\right\}}
$$

such that

$$
f(g u)=u_{\mathrm{S}(\mathrm{B}) \cup \mathrm{R} \cup \mathrm{S}_{l}}^{-1} f(g)
$$

for all $u \in \mathrm{U}$ and $g \in \mathrm{G}\left(\mathbf{A}_{\mathrm{F}^{+}}^{\infty}\right)$. Here $u_{\mathrm{S}(\mathrm{B}) \cup \mathrm{R} \cup \mathrm{S}_{l}}$ denotes the projection of $u$ to $\mathrm{G}\left(\mathrm{F}_{l}^{+}\right) \times \prod_{v \in \mathrm{S}(\mathrm{B})} \mathrm{G}\left(\mathrm{F}_{v}^{+}\right) \times \prod_{v \in \mathrm{R}} \mathrm{Iw}(\widetilde{v})$. If $\mathrm{A}$ is a $\mathscr{O}$-module we have

$$
\mathrm{S}_{a,\left\{\rho_{v}\right\},\left\{\chi_{v}\right\}}(\mathrm{U}, \mathrm{A})=\mathrm{S}_{a,\left\{\rho_{v}\right\},\left\{\chi_{v}\right\}}(\mathrm{U}, \mathscr{O}) \otimes_{\mathscr{O}} \mathrm{A}
$$

(because $\mathrm{U}$ is sufficiently small). We make an exactly analogous definition with $\mathrm{U}_{0}(\mathrm{Q})$ or $\mathrm{U}_{1}(\mathrm{Q})$ replacing $\mathrm{U}$. These spaces satisfy the same base change property as $\mathrm{U}_{0}(\mathrm{Q})$ and $\mathrm{U}_{1}(\mathrm{Q})$ are also sufficiently small. (See Section 3.3 of [CHT] for details.)

We will denote by

$$
\mathbf{T}_{a,\left\{\rho_{v}\right\},\left\{\chi_{v}\right\}}^{\mathrm{T}}(\mathrm{U})
$$

the $\mathscr{O}$-subalgebra of $\operatorname{End}\left(\mathrm{S}_{a,\left\{\rho_{v}\right\},\left\{\chi_{v}\right\}}(\mathrm{U}, \mathscr{O})\right)$ generated by the Hecke operators

$$
\mathrm{T}_{w}^{(j)}=i_{w}^{-1}\left(\mathrm{GL}_{n}\left(\mathscr{O}_{\mathrm{F}, w}\right)\left(\begin{array}{cc}
\varpi_{w} 1_{j} & 0 \\
0 & 1_{n-j}
\end{array}\right) \mathrm{GL}_{n}\left(\mathscr{O}_{\mathrm{F}, w}\right)\right) \times \mathrm{U}^{v}
$$

for $j=1, \ldots, n$ and $\left(\mathrm{T}_{w}^{(n)}\right)^{-1}$, where $w$ runs over places of $\mathrm{F}$ which are split over a place $v \notin \mathrm{T}$ of $\mathrm{F}^{+}$. (Note that $\mathrm{T}_{w}^{(j)}=\left(\mathrm{T}_{w}^{(n)}\right)^{-1} \mathrm{~T}_{w}^{(n-j)}$, so we need only consider one place $w$ above a given place $v$ of $\mathrm{F}^{+}$.) The algebra $\mathbf{T}_{a,\left\{\rho_{v}\right\},\left\{\chi_{v}\right\}}^{\mathrm{T}}(\mathrm{U})$ is reduced (see Corollary 3.3 .3 of $[\mathrm{CHT}]$ ) and finite, free as a $\mathscr{O}$-module. Again we make an exactly analogous definition with $\mathrm{U}$ replaced by $\mathrm{U}_{0}(\mathrm{Q})$ or $\mathrm{U}_{1}(\mathrm{Q})$ and $\mathrm{T}$ replaced 
by $\mathrm{T}(\mathrm{Q})$. The algebras $\mathbf{T}_{a,\left\{\rho_{v}\right\},\left\{\chi_{v}\right\}}^{\mathrm{T}}\left(\mathrm{U}_{0}(\mathrm{Q})\right)$ and $\mathbf{T}_{a,\left\{\rho_{v}\right\},\left\{\chi_{v}\right\}}^{\mathrm{T}(\mathrm{Q})}\left(\mathrm{U}_{1}(\mathrm{Q})\right)$ are also reduced and finite free as $\mathscr{O}$-modules.

We now turn to some Galois theory. Recall that in [CHT] we defined a group scheme $\mathscr{G}_{n}$ over $\mathbf{Z}$ to be the semi-direct product of $\mathrm{GL}_{n} \times \mathrm{GL}_{1}$ by the group $\{1, j\}$ acting on $\mathrm{GL}_{n} \times \mathrm{GL}_{1}$ by

$$
J(g, \mu) J^{-1}=\left(\mu^{t} g^{-1}, \mu\right) .
$$

There is a homomorphism $v: \mathscr{G}_{n} \rightarrow \mathrm{GL}_{1}$ which sends $(g, \mu)$ to $\mu$ and $j$ to -1 . We also defined a subgroup $\mathrm{H} \subset \mathscr{G}_{n}(k)$ to be big if the following conditions are satisfied.

- $\mathrm{H} \cap \mathscr{G}_{n}^{0}(k)$ has no $l$-power order quotient.

$-\mathrm{H}^{0}\left(\mathrm{H}, \mathfrak{g}_{n}(k)\right)=(0)$.

$-\mathrm{H}^{1}\left(\mathrm{H}, \mathfrak{g}_{n}(k)\right)=(0)$.

- For all irreducible $k[\mathrm{H}]$-submodules $\mathrm{W}$ of $\mathfrak{g}_{n}(k)$ we can find $h \in \mathrm{H} \cap \mathscr{G}_{n}^{0}(k)$ and $\alpha \in k$ with the following properties. The $\alpha$ generalised eigenspace $\mathrm{V}_{h, \alpha}$ of $h$ in $k^{n}$ is one dimensional. Let $\pi_{h, \alpha}: k^{n} \rightarrow \mathrm{V}_{h, \alpha}$ (resp. $i_{h, \alpha}$ ) denote the $h$-equivariant projection of $k^{n}$ to $\mathrm{V}_{h, \alpha}$ (resp. $h$-equivariant injection of $\mathrm{V}_{h, \alpha}$ into $k^{n}$. Then $\pi_{h, \alpha} \circ \mathrm{W} \circ i_{h, \alpha} \neq(0)$.

(See Section 2.5 of [CHT] for this and for some examples of big subgroups.)

Let $\mathfrak{m}$ be a maximal ideal of $\mathbf{T}_{a,\left\{\rho_{v}\right\},\{1\}}^{\mathrm{T}}(\mathrm{U})$ with residue field $k$ and let

$$
\bar{r}_{\mathfrak{m}}: \operatorname{Gal}(\overline{\mathrm{F}} / \mathrm{F}) \longrightarrow \mathrm{GL}_{n}(k)
$$

be a continuous homomorphism associated to $\mathfrak{m}$ as in Proposition 3.4.2 of [CHT]. Thus if $w$ is a prime of $\mathrm{F}$ split over a prime $v \notin \mathrm{T}$ of $\mathrm{F}^{+}$then $\bar{r}_{\mathfrak{m}}$ is unramified at $w$ and $\bar{r}_{\mathfrak{m}}\left(\right.$ Frob $\left._{w}\right)$ has characteristic polynomial

$$
\begin{aligned}
\mathrm{X}^{n}-\mathrm{T}_{w}^{(1)} \mathrm{X}^{n-1} & +\ldots+(-1)^{j}(\mathbf{N} w)^{j(j-1) / 2} \mathrm{~T}_{w}^{(j)} \mathrm{X}^{n-j} \\
& +\ldots+(-1)^{n}(\mathbf{N} w)^{n(n-1) / 2} \mathrm{~T}_{w}^{(n)} .
\end{aligned}
$$

We will assume that the representation $\bar{r}_{\mathfrak{m}}$ is absolutely irreducible, i.e. that $\mathfrak{m}$ is non-Eisenstein. Then by Proposition 3.4.4 of [CHT] $\bar{r}_{\mathfrak{m}}$ extends to a continuous homomorphism

$$
\bar{r}_{\mathfrak{m}}: \operatorname{Gal}\left(\overline{\mathrm{F}} / \mathrm{F}^{+}\right) \longrightarrow \mathscr{G}_{n}(k)
$$

with

$$
\nu \circ \bar{r}_{\mathfrak{m}}=\epsilon^{1-n} \delta_{\mathrm{F} / \mathrm{F}^{+}}^{\mu_{\mathfrak{m}}}
$$

where $\delta_{\mathrm{F} / \mathrm{F}^{+}}$is the non-trivial character of $\mathrm{Gal}\left(\mathrm{F} / \mathrm{F}^{+}\right)$and where $\mu_{\mathfrak{m}} \in \mathbf{Z} / 2 \mathbf{Z}$. We will assume that $\bar{r}_{\mathfrak{m}}$ has the following properties. 
$-\bar{r}_{\mathfrak{m}}\left(\mathrm{Gal}\left(\overline{\mathrm{F}} / \mathrm{F}^{+}\left(\zeta_{l}\right)\right)\right)$ is big.

- If $v \in \mathrm{R}$ then $\bar{r}_{\mathfrak{m}}\left(\mathrm{G}_{\mathrm{F}_{\widetilde{v}}}\right)=\left\{1_{n}\right\}$.

- If $v \in \mathrm{S}_{a}$ then $\bar{r}_{\mathfrak{m}}$ is unramified at $v$ and

$$
\mathrm{H}^{0}\left(\operatorname{Gal}\left(\overline{\mathrm{F}}_{\widetilde{v}} / \mathrm{F}_{\widetilde{v}}\right),\left(\operatorname{ad} \bar{r}_{\mathfrak{m}}\right)(1)\right)=(0) .
$$

- If $v \in \mathrm{S}(\mathrm{B})_{1}$ then $\bar{r}_{\mathfrak{m}}\left(\mathrm{Gal}\left(\overline{\mathrm{F}}_{\widetilde{v}} / \mathrm{F}_{\widetilde{v}}\right)\right)=\{1\}$.

Set $\mathbf{T}=\mathbf{T}_{a,\left\{\rho_{v}\right\},\{1\}}^{\mathrm{T}}(\mathrm{U})_{\mathfrak{m}}$ and $\mathrm{H}=\mathrm{S}_{a,\left\{\rho_{v}\right\},\{1\}}(\mathrm{U}, \mathscr{O})_{\mathfrak{m}}$. By Proposition 3.4.4 of $[\mathrm{CHT}]$ there is a continuous representation

$$
r_{\mathfrak{m}}: \mathrm{G}_{\mathrm{F}^{+}} \longrightarrow \mathscr{G}_{n}(\mathbf{T})
$$

lifting $\bar{r}_{\mathfrak{m}}$ and such that if $w$ is a prime of $\mathrm{F}$ split over a prime $v \notin \mathrm{T}$ of $\mathrm{F}^{+}$then $r_{\mathfrak{m}}$ is unramified at $w$ and $r_{\mathfrak{m}}\left(\right.$ Frob $\left._{w}\right)$ has characteristic polynomial

$$
\begin{aligned}
\mathrm{X}^{n}-\mathrm{T}_{w}^{(1)} \mathrm{X}^{n-1} & +\ldots+(-1)^{j}(\mathbf{N} w)^{j(j-1) / 2} \mathrm{~T}_{w}^{(j)} \mathrm{X}^{n-j} \\
& +\ldots+(-1)^{n}(\mathbf{N} w)^{n(n-1) / 2} \mathrm{~T}_{w}^{(n)} .
\end{aligned}
$$

For $v \in \mathrm{T}$ we write

$$
r_{v}^{\text {univ }}: \mathrm{G}_{\widetilde{v}} \longrightarrow \mathrm{GL}_{n}\left(\mathrm{R}_{v}^{\text {loc }}\right)
$$

for the universal lifting (not deformation, i.e. it parametrises lifts not conjugacy classes of lifts) of $\left.\bar{r}_{\mathfrak{m}}\right|_{\mathrm{G}_{\tilde{r}}}$. (See Section 2.2 of [CHT].) Consider the deformation problem $\mathscr{S}$ given by

$$
\left(\mathrm{F} / \mathrm{F}^{+}, \mathrm{T}, \widetilde{\mathrm{T}}, \mathscr{O}, \bar{r}_{\mathfrak{m}}, \epsilon^{1-n} \delta_{\mathrm{F} / \mathrm{F}^{+}}^{\mu_{\mathfrak{m}}},\left\{\mathscr{D}_{v}\right\}_{v \in \mathrm{T}}\right)
$$

and the ideals $\mathscr{I}_{v}$ of $\mathrm{R}_{v}^{\text {loc }}$ corresponding to $\mathscr{D}_{v}$, where:

- For $v \in \mathrm{S}_{a}, \mathscr{D}_{v}$ will consist of all lifts of $\left.\bar{r}_{\mathfrak{m}}\right|_{\mathrm{Gal}\left(\overline{\mathrm{F}}_{\tilde{v}} / \mathrm{F}_{\tilde{v}}\right)}$ and $\mathscr{I}_{v}=(0)$.

- For $v \in \mathrm{S}_{l}, \mathscr{D}_{v}$ and $\mathscr{I}_{v}$ will be as described in Section 2.4.1 of [CHT] (i.e. consists of crystalline deformations).

- If $v \in \mathrm{S}(\mathrm{B})_{1}$ then $\mathscr{D}_{v}$ will correspond to $\mathscr{I}_{v}=\mathscr{I}_{v}^{\text {Stein }}$.

- If $v \in \mathrm{S}(\mathrm{B})_{2}$ then $\mathscr{D}_{v}$ will consist of lifts which are $\tilde{r}_{\widetilde{v}}$-discrete series as described in Section 2.4.5 of [CHT].

- For $v \in \mathrm{R}$ then $\mathscr{D}_{v}$ corresponds to the ideal $\mathscr{I}_{v}=\mathscr{I}_{v}^{(1, \ldots, 1)}$ of $\mathrm{R}_{v}^{\text {loc }}$.

Also let

$$
r_{\mathscr{S}}^{\text {univ }}: \operatorname{Gal}\left(\overline{\mathrm{F}} / \mathrm{F}^{+}\right) \longrightarrow \mathscr{G}_{n}\left(\mathrm{R}_{\mathscr{S}}^{\text {univ }}\right)
$$

denote the universal deformation of $\bar{r}_{\mathfrak{m}}$ of type $\mathscr{S}$. (Again see Section 2.2 of [CHT].) By Proposition 3.4.4 of [CHT] the representation $r_{\mathfrak{m}}$ gives rise to a surjection

$$
\mathrm{R}_{\mathscr{S}}^{\text {univ }} \rightarrow \mathbf{T}
$$

which makes $\mathrm{H}$ an $\mathrm{R}_{\mathscr{S}}^{\text {univ }}$-module. 
We can now state our main result.

Theorem 4.1. - Keep the notation and assumptions of the start of this section. Then $\left(\mathrm{R}_{\mathscr{S}}^{\text {univ }}\right)^{\text {red }} \stackrel{\sim}{\longrightarrow} \mathbf{T}$.

Moreover $\mu_{\mathfrak{m}} \equiv n \bmod 2$.

Proof. - The proof will involve the consideration of various other deformation problems and the corresponding Hecke algebras and spaces of modular forms. More specifically we will consider sets of characters

$$
\left\{\chi_{v}: \operatorname{Iw}(\widetilde{v}) / \operatorname{Iw}_{1}(\widetilde{v}) \rightarrow \mathscr{O}^{\times}\right\}_{v \in \mathrm{R}}
$$

of order dividing $l$, and finite sets $\mathrm{Q}$ of primes of $\mathrm{F}^{+}$disjoint from $\mathrm{T}$ and such that if $v \in Q$ then

$-v$ splits $w w^{c}$ in $\mathrm{F}$,

$-\mathbf{N} v \equiv 1 \bmod l$, and

$-\left.\bar{r}_{\mathfrak{m}}\right|_{\mathrm{G}_{\mathrm{F}}}=\bar{\psi}_{w} \oplus \bar{s}_{w}$, with $\operatorname{dim} \bar{\psi}_{w}=1$ and $\bar{\psi}_{w}$ not isomorphic to any subquotient of $\bar{s}_{w}$.

We must first assemble some notations.

Consider the deformation problem $\mathscr{S}_{\left\{\chi_{v}\right\}, Q}$ given by

$$
\left(\mathrm{F} / \mathrm{F}^{+}, \mathrm{T}(\mathrm{Q}), \widetilde{\mathrm{T}}(\mathrm{Q}), \mathscr{O}, \bar{r}_{\mathfrak{m}}, \epsilon^{1-n} \delta_{\mathrm{F} / \mathrm{F}^{+}}^{\mu_{\mathfrak{m}}},\left\{\mathscr{D}_{v}\right\}_{v \in \mathrm{T}(\mathrm{Q})}\right)
$$

and the ideals $\mathscr{I}_{v}$ of $\mathrm{R}_{v}^{\text {loc }}$ corresponding to $\mathscr{D}_{v}$, where:

- For $v \in \mathrm{S}_{a}, \mathscr{D}_{v}$ will consist of all lifts of $\left.\bar{r}_{\mathfrak{m}}\right|_{\mathrm{Gal}\left(\overline{\mathrm{F}}_{\widetilde{v}} / \mathrm{F}_{\tilde{v}}\right)}$ and $\mathscr{I}_{v}=(0)$.

- For $v \in \mathrm{S}_{l}, \mathscr{D}_{v}$ and $\mathscr{I}_{v}$ will be as described in Section 2.4.1 of [CHT] (i.e. consists of crystalline deformations).

- If $v \in \mathrm{S}(\mathrm{B})_{1}$ then $\mathscr{D}_{v}$ will correspond to $\mathscr{I}_{v}=\mathscr{I}_{v}^{\text {Stein }}$.

- If $v \in \mathrm{S}(\mathrm{B})_{2}$ then $\mathscr{D}_{v}$ will consist of lifts which are $\tilde{r}_{\tilde{v}}$-discrete series as described in Section 2.4.5 of [CHT].

- For $v \in \mathrm{R}$ then $\mathscr{D}_{v}$ corresponds to the ideal $\mathscr{I}_{v}=\mathscr{I}_{v}^{\left(\chi_{v, 1}, \ldots, \chi_{v, n}\right)}$ of $\mathrm{R}_{v}^{\text {loc }}$.

- For $v \in \mathrm{Q}$ then $\mathscr{D}_{v}$ corresponds to the maximal quotient $\mathrm{R}_{v}^{\text {loc }} / \mathscr{I}_{v}$ of $\mathrm{R}_{v}^{\text {loc }}$ over which $r_{v}^{\text {univ }}$ becomes equivalent to $\psi_{v} \oplus s_{v}$ where $\psi$ lifts $\bar{\psi}_{v}$ and where $s$ is unramified and lifts $\bar{s}_{v}$. (See Section 2.4.6 of [CHT].)

(For $v \in \mathrm{R}$ the sets $\mathscr{D}_{v}$ and the ideals $\mathscr{I}_{v}$ depend on $\chi_{v}$, but we suppress this in the notation.) Let

$$
r_{\mathscr{S}_{\left\{x_{\vartheta}\right\}, Q}}^{\text {univ }}: \operatorname{Gal}\left(\overline{\mathrm{F}} / \mathrm{F}^{+}\right) \longrightarrow \mathscr{G}_{n}\left(\mathrm{R}_{\mathscr{S}_{\left\{x_{v}\right\}, Q}^{\text {univ }}}\right)
$$


denote the universal deformation of $\bar{r}_{\mathfrak{m}}$ of type $\mathscr{S}_{\left\{\chi_{v}\right\}, \mathcal{Q}}$, and let

$$
r_{\mathscr{S}_{\left\{x_{v}\right\}, Q}}^{\square_{\mathrm{T}}}: \operatorname{Gal}\left(\overline{\mathrm{F}} / \mathrm{F}^{+}\right) \longrightarrow \mathscr{G}_{n}\left(\mathrm{R}_{\mathscr{S}_{\left\{x_{v}\right\}, Q}}^{\square_{\mathrm{T}}}\right)
$$

denote the universal deformation of $\bar{r}_{\mathfrak{m}}$ of type $\mathscr{S}_{\left\{\chi_{v}\right\}, \mathcal{Q}}$ framed at all $v \in \mathrm{T}$. Thus $\mathrm{R}_{\mathscr{S}\{1\}, \emptyset}^{\text {univ }}=\mathrm{R}_{\mathscr{S}}^{\text {univ }}$. (See Section 2.2 of $[\mathrm{CHT}]$.) Note that

$$
\mathrm{R}_{\mathscr{S}_{\left\{x_{v}\right\}, Q}^{\text {univ }}} / \lambda \cong \mathrm{R}_{\mathscr{S}_{\{1\}, Q}}^{\text {univ }} / \lambda
$$

and

$$
\mathrm{R}_{\mathscr{S}_{\left\{\chi_{v}\right\}, Q}}^{\square_{\mathrm{T}}} / \lambda \cong \mathrm{R}_{\mathscr{S}_{\{1\}, Q}}^{\square_{\mathrm{T}}} / \lambda
$$

compatibly with the natural maps

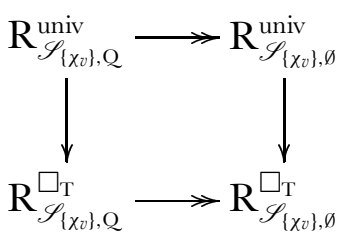

and

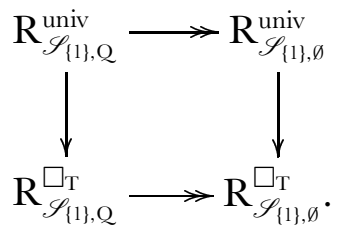

Let

$$
\mathrm{R}_{\left\{\chi_{v}\right\}, \mathrm{T}}^{\mathrm{loc}}=\bigotimes_{v \in \mathrm{T}} \mathrm{R}_{v}^{\mathrm{loc}} / \mathscr{I}_{v}
$$

so that again

$$
\mathrm{R}_{\left\{\chi_{v}\right\}, \mathrm{T}}^{\mathrm{loc}} / \lambda \cong \mathrm{R}_{\{1\}, \mathrm{T}}^{\mathrm{loc}} / \lambda
$$

There are natural maps

$$
\mathrm{R}_{\left\{\chi_{v}\right\}, \mathrm{T}}^{\mathrm{loc}} \longrightarrow \mathrm{R}_{\mathscr{S}_{\left\{\chi_{v}\right\}, \mathrm{Q}}}^{\square_{\mathrm{T}}}
$$

which modulo $\lambda$ are compatible with the identifications $\mathrm{R}_{\left\{\chi_{v}\right\}, \mathrm{T}}^{\mathrm{loc}} / \lambda \cong \mathrm{R}_{\{1\}, \mathrm{T}}^{\mathrm{loc}} / \lambda$ and $\mathrm{R}_{\mathscr{S}_{\left\{x_{v}\right\}, Q}}^{\square_{\mathrm{T}}} / \lambda \cong \mathrm{R}_{\mathscr{S}_{\{1\}, Q}}^{\square_{\mathrm{T}}} / \lambda$. (See Section 2.2 of $[\mathrm{CHT}]$.) 
(The sets $\mathrm{Q}$ will be the auxiliary primes needed for the Taylor-Wiles method (see [TW]). To handle non-minimal deformations we will work simultaneously with $\mathrm{R}_{\mathscr{S}}^{\text {univ }}$ and $\mathrm{R}_{\mathscr{S}_{\left\{\chi_{v}\right\}, \emptyset}}$ for suitably chosen characters $\left\{\chi_{v}\right\}_{v \in \mathrm{S}}$. The Taylor-Wiles method

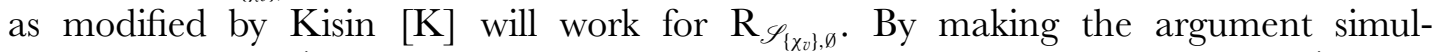
taneously for $\mathrm{R}_{\mathscr{S}}^{\text {univ }}$ and $\mathrm{R}_{\mathscr{S}_{\left\{\chi_{v}\right\}, \emptyset}}$ we will be able to finesse the result for $\mathrm{R}_{\mathscr{S}}^{\text {univ }}$ from the $\mathrm{R}_{\mathscr{I}_{\left\{x_{0}\right\}, \emptyset} \text { case.) }}$

Write

$$
\mathscr{T}=\mathscr{O}\left[\left[\mathrm{X}_{v, i, j}: \quad v \in \mathrm{T}, i, j=1, \ldots, n\right]\right] .
$$

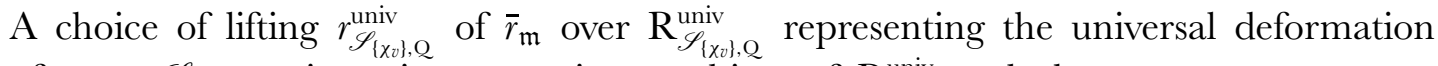
of type $\mathscr{S}_{\left\{\chi_{v}\right\}, \mathrm{Q}}$ gives rise to an isomorphism of $\mathrm{R}_{\mathscr{S}_{\left\{\chi_{v}\right\}, Q}^{\text {univ }}}^{\text {-algebras }}$

$$
\mathrm{R}_{\mathscr{T}_{\left\{x_{v}\right\}, Q}}^{\square_{\mathrm{T}}} \stackrel{\sim}{\longrightarrow} \mathrm{R}_{\mathscr{S}_{\left\{x_{v}\right\}, Q}}^{\text {univ }} \widehat{\otimes}_{\mathscr{O}} \mathscr{T}
$$

so that

$$
\left(r_{\mathscr{S}_{\left\{x_{v}\right\}, Q}^{\text {univ }}} ;\left\{1_{n}+\left(\mathrm{X}_{v, i, j}\right)\right\}_{v \in \mathrm{T}}\right)
$$

is the universal framed deformation. (See Section 2.2 of [CHT].) We can, and will, choose the lifting $r_{\mathscr{S}_{\left\{x_{0}\right\}, Q}}^{\text {univ }}$ so that

$$
r_{\mathscr{S}_{\left\{x_{0}\right\}, Q}}^{\text {univ }} \otimes_{\mathscr{O}} k=r_{\mathscr{S}_{\{1\}, Q}}^{\text {univ }} \otimes_{\mathscr{O}} k
$$

under the natural identification $\mathrm{R}_{\mathscr{S}_{\left\{x_{v}\right\}, Q}^{\text {univ }}} / \lambda \cong \mathrm{R}_{\mathscr{S}_{\{1\}, Q}}^{\text {univ }} / \lambda$. Then the isomorphisms

$$
\mathrm{R}_{\mathscr{I}_{\left\{x_{v}\right\}, Q}}^{\square_{\mathrm{T}}} \stackrel{\sim}{\longrightarrow} \mathrm{R}_{\mathscr{I}_{\left\{x_{v}\right\}, Q}}^{\text {univ }} \widehat{\otimes}_{\mathscr{O}} \mathscr{T}
$$

and

$$
\mathrm{R}_{\mathscr{S}_{\{1\}, Q}}^{\square_{\mathrm{T}}} \stackrel{\sim}{\longrightarrow} \mathrm{R}_{\mathscr{S}_{\{1\}, Q}}^{\text {univ }} \widehat{\otimes}_{\mathscr{O}} \mathscr{T}
$$

are compatible with the identification $\mathrm{R}_{\mathscr{S}_{\{x\}\}, Q}}^{\square_{\mathrm{T}}} / \lambda \cong \mathrm{R}_{\mathscr{S}_{\{1\}, Q}}^{\square_{\mathrm{T}}} / \lambda$ and the identification $\mathrm{R}_{\mathscr{S}_{\left\{x_{i}\right\}, Q}}^{\text {univ }} / \lambda \cong \mathrm{R}_{\mathscr{S}_{\{1\}, Q}}^{\text {univ }} / \lambda$.

Also write $\Delta_{\mathrm{Q}}$ for the maximal $l$-power quotient of $\prod_{v \in \mathrm{Q}} k(v)^{\times}$and let $\mathfrak{a}_{\mathrm{Q}}$ denote the ideal of $\mathscr{T}\left[\Delta_{\mathrm{Q}}\right]$ generated by the augmentation ideal of $\mathscr{O}\left[\Delta_{\mathrm{Q}}\right]$ and by the $\mathrm{X}_{v, i, j}$ for $v \in \mathrm{T}$ and $i, j=1, \ldots, n$. Then

$$
\prod_{v \in \mathrm{Q}}\left(\psi_{v} \circ \operatorname{Art}_{\mathbb{F}_{\tilde{v}}}\right): \Delta_{\mathrm{Q}} \longrightarrow\left(\mathrm{R}_{\mathscr{S}_{\left\{\chi_{v}\right\}, \mathrm{Q}}}^{\text {univ }}\right)^{\times}
$$

makes $\mathrm{R}_{\mathscr{S}_{\left\{\chi_{v}\right\}, \mathrm{Q}}}^{\text {univ }}$ an $\mathscr{T}\left[\Delta_{\mathrm{Q}}\right]$-algebra. This $\mathscr{T}\left[\Delta_{\mathrm{Q}}\right]$-algebra structure is compatible with the identification $\mathrm{R}_{\mathscr{S}_{\left\{x_{v}\right\}, Q}}^{\text {univ }} / \lambda \cong \mathrm{R}_{\mathscr{S}_{\{1\}, Q}}^{\text {univ }} / \lambda$, because the liftings $r_{\mathscr{S}_{\left\{x_{v}\right\}, Q}}^{\square_{\mathrm{T}}}$ and $r_{\mathscr{S}_{\{1\}, Q}}^{\square_{\mathrm{T}}}$ 
were chosen compatibly. We also obtain isomorphisms of $\mathrm{R}_{\left\{\chi_{v}\right\}, \mathrm{T}^{\text {loc }}}^{\mathrm{lalgebras}}$

$$
\mathrm{R}_{\mathscr{S}_{\{x v\}, Q}}^{\square_{\mathrm{T}}} / \mathfrak{a}_{\mathrm{Q}} \stackrel{\sim}{\longrightarrow} \mathrm{R}_{\mathscr{S}_{\{x v\}, \emptyset\}}^{\text {univ }}}
$$

compatibly with the identifications $\mathrm{R}_{\mathscr{S}_{\left\{x_{0}\right\}, Q}}^{\square_{\mathrm{T}}} / \lambda \cong \mathrm{R}_{\mathscr{S}_{\{1\}, Q}}^{\square_{\mathrm{T}}} / \lambda$ and $\mathrm{R}_{\mathscr{S}_{\left\{x_{x}\right\}, \emptyset}}^{\text {univ }} / \lambda \cong \mathrm{R}_{\mathscr{S}_{\{1\}, \emptyset}}^{\text {univ }} / \lambda$.

We have

$$
\mathrm{S}_{a,\left\{\rho_{v}\right\},\{1\}}(\mathrm{U}, k)=\mathrm{S}_{a,\left\{\rho_{v}\right\},\left\{\chi_{v}\right\}}(\mathrm{U}, k) \hookrightarrow \mathrm{S}_{a,\left\{\rho_{v}\right\},\left\{\chi_{v}\right\}}\left(\mathrm{U}_{1}(\mathrm{Q}), k\right) .
$$

Thus there is a maximal ideal $\mathfrak{m}_{\left\{\chi_{v}\right\}, \mathcal{Q}}$ of $\mathbf{T}_{a,\left\{\rho_{v}\right\},\left\{\chi_{v}\right\}}^{\mathrm{T}(\mathrm{Q})}\left(\mathrm{U}_{1}(\mathrm{Q})\right)$ with residue field $k$ such that for a prime $w$ of $\mathrm{F}$ split over a prime $v \notin \mathrm{T}(\mathrm{Q})$ of $\mathrm{F}^{+}$the Hecke operators $\mathrm{T}_{w}^{(i)}$ have the same image in $\mathbf{T}_{a,\left\{\rho_{v}\right\},\left\{\chi_{v}\right\}}^{\mathrm{T}(\mathrm{Q})}\left(\mathrm{U}_{1}(\mathrm{Q})\right) / \mathfrak{m}_{\left\{\chi_{v}\right\}, \mathrm{Q}}=k$ as in $\mathbf{T}_{a,\left\{\rho_{v}\right\},\{1\}}^{\mathrm{T}(\mathrm{Q})}(\mathrm{U}) / \mathfrak{m}=k$. By Proposition 3.4.4 of [CHT] there is a continuous representation

$$
r_{\mathfrak{m}_{\left\{\chi_{v}\right\}, Q}}: \mathrm{G}_{\mathrm{F}^{+}} \longrightarrow \mathscr{G}_{n}\left(\mathbf{T}_{a,\left\{\rho_{v}\right\},\left\{\chi_{v}\right\}}^{\mathrm{T}(\mathrm{Q})}\left(\mathrm{U}_{1}(\mathrm{Q})\right)_{\mathfrak{m}_{\left\{\chi_{v}\right\}, Q}}\right)
$$

lifting $\bar{r}_{\mathfrak{m}}$ and such that if $w$ is a prime of $\mathrm{F}$ split over a prime $v \notin \mathrm{T}(\mathrm{Q})$ of $\mathrm{F}^{+}$ then $r_{\mathfrak{m}_{\left\{\chi_{v}\right\}, Q}}$ is unramified at $w$ and $r_{\mathfrak{m}_{\left\{\chi_{v}\right\}, Q}}\left(\mathrm{Frob}_{w}\right)$ has characteristic polynomial

$$
\begin{aligned}
\mathrm{X}^{n}-\mathrm{T}_{w}^{(1)} \mathrm{X}^{n-1} & +\ldots+(-1)^{j}(\mathbf{N} w)^{j(j-1) / 2} \mathrm{~T}_{w}^{(j)} \mathrm{X}^{n-j} \\
& +\ldots+(-1)^{n}(\mathbf{N} w)^{n(n-1) / 2} \mathrm{~T}_{w}^{(n)} .
\end{aligned}
$$

Set $\mathbf{T}_{\left\{\chi_{v}\right\}}=\mathbf{T}_{a,\left\{\rho_{v}\right\},\left\{\chi_{v}\right\}}^{\mathrm{T}}(\mathrm{U})_{\mathfrak{m}_{\left\{\chi_{v}\right\}, \emptyset}}$ and $\mathrm{H}_{\left\{\chi_{v}\right\}}=\mathrm{S}_{a,\left\{\rho_{v}\right\},\left\{\chi_{v}\right\}}(\mathrm{U}, \mathscr{O})_{\mathfrak{m}_{\left\{\chi_{v}\right\}, \emptyset}}$. By Corollary 3.4 .5 of $[\mathrm{CHT}]$ we see that we have

$$
\mathbf{T}_{a,\left\{\rho_{v}\right\},\left\{\chi_{v}\right\}}^{\mathrm{T}(\mathrm{Q})}\left(\mathrm{U}_{1}(\mathrm{Q})\right)_{\mathbf{m}_{\left\{x_{v}\right\}, Q}} \rightarrow \mathbf{T}_{a,\left\{\rho_{v}\right\},\left\{\chi_{v}\right\}}^{\mathrm{T}(\mathrm{Q})}\left(\mathrm{U}_{0}(\mathrm{Q})\right)_{\mathfrak{m}_{\left\{x_{v}\right\}, Q}} \rightarrow \mathbf{T}_{a,\left\{\rho_{v}\right\},\left\{\chi_{v}\right\}}^{\mathrm{T}(\mathrm{Q})}(\mathrm{U})_{\mathfrak{m}_{\left\{x_{v}\right\}, Q}}=\mathbf{T}_{\left\{x_{v}\right\}} .
$$

For $v \in Q$ choose $\phi_{\widetilde{v}} \in \operatorname{Gal}\left(\overline{\mathrm{F}}_{\widetilde{v}} / \mathrm{F}_{\widetilde{v}}\right)$ lifting $\operatorname{Frob}_{\widetilde{v}}$ and $\varpi_{\widetilde{v}} \in \mathrm{F}_{\widetilde{v}}^{\times}$with $\phi_{\widetilde{v}}=\operatorname{Art}_{\mathrm{F}_{\widetilde{v}}} \varpi_{\widetilde{v}}$ on the maximal abelian extension of $\mathrm{F}_{\widetilde{v}}$. Let

$$
\mathrm{P}_{\widetilde{v}} \in \mathbf{T}_{a,\left\{\rho_{v}\right\},\left\{\chi_{v}\right\}}^{\mathrm{T}(\mathrm{Q})}\left(\mathrm{U}_{1}(\mathrm{Q})\right)_{\mathfrak{m}_{\left\{\chi_{v}\right\}, Q}}[\mathrm{X}]
$$

denote the characteristic polynomial of $r_{\mathfrak{m}_{\left\{\chi_{v}\right\}, Q}}\left(\phi_{\widetilde{v}}\right)$. By Hensel's lemma we have a unique factorisation

$$
\mathrm{P}_{\widetilde{v}}(\mathrm{X})=\left(\mathrm{X}-\mathrm{A}_{\widetilde{v}}\right) \mathrm{Q}_{\widetilde{v}}(\mathrm{X})
$$

over $\mathbf{T}_{a,\left\{\rho_{v}\right\},\left\{\chi_{v}\right\}}^{\mathrm{T}(\mathrm{Q})}\left(\mathrm{U}_{1}(\mathrm{Q})\right)_{\mathfrak{m}_{\left\{\chi_{v}\right\}, Q}}$, where $\mathrm{A}_{\widetilde{v}}$ lifts $\bar{\psi}_{v}\left(\phi_{\widetilde{v}}\right)$ and

$$
\mathrm{Q}_{\widetilde{v}}\left(\mathrm{~A}_{\widetilde{v}}\right) \in \mathbf{T}_{a,\left\{\rho_{v}\right\},\left\{\chi_{v}\right\}}^{\mathrm{T}(\mathrm{Q})}\left(\mathrm{U}_{1}(\mathrm{Q})\right)_{\mathfrak{m}_{\left\{\chi_{v}\right\}, \mathrm{Q}}}^{\times} .
$$

If $v \in \mathrm{Q}$ and $\alpha \in \mathrm{F}_{\widetilde{v}}$ write

$$
\mathrm{V}_{\alpha}=i_{\widetilde{v}}^{-1}\left(\mathrm{U}_{1}(\widetilde{v})\left(\begin{array}{cc}
1_{n-1} & 0 \\
0 & \alpha
\end{array}\right) \mathrm{U}_{1}(\widetilde{v})\right) \times \mathrm{U}^{v}
$$


(See Section 3.4 of [CHT] for details.) Using Lemmas 3.1.3 and 3.1.5 of [CHT] we see that $\mathrm{P}_{\widetilde{v}}\left(\mathrm{~V}_{\varpi_{t^{v}}}\right)=0$ on $\mathrm{S}_{a,\left\{\rho_{v}\right\},\left\{\chi_{v}\right\}}\left(\mathrm{U}_{1}(\mathrm{Q}), \mathscr{O}\right)_{\mathfrak{m}_{\left\{x_{v}\right\}, Q}}$. Set

$$
\mathrm{H}_{1,\left\{\chi_{v}\right\}, \mathcal{Q}}=\left(\prod_{v \in \mathcal{Q}} \mathrm{Q}_{\widetilde{v}}\left(\mathrm{~V}_{\varpi_{\tilde{v}}}\right)\right) \mathrm{S}_{a,\left\{\rho_{v}\right\},\left\{\chi_{v}\right\}}\left(\mathrm{U}_{1}(\mathrm{Q}), \mathscr{O}\right)_{\mathfrak{m}_{\left\{\chi_{v}\right\}, \mathcal{Q}}}
$$

and

$$
\mathrm{H}_{0,\left\{\chi_{v}\right\}, \mathcal{Q}}=\left(\prod_{v \in \mathcal{Q}} \mathrm{Q}_{\widetilde{v}}\left(\mathrm{~V}_{\varpi \widetilde{v}}\right)\right) \mathrm{S}_{a,\left\{\rho_{v}\right\},\left\{\chi_{v}\right\}}\left(\mathrm{U}_{0}(\mathrm{Q}), \mathscr{O}\right)_{\mathfrak{m}_{\left\{\chi_{v}\right\}, Q}}
$$

We see that $\mathrm{H}_{1,\left\{\chi_{v}\right\}, Q}$ is a $\mathbf{T}_{a,\left\{\rho_{v}\right\},\left\{\chi_{v}\right\}}^{\mathrm{T}(\mathrm{Q})}\left(\mathrm{U}_{1}(\mathrm{Q})\right)$-direct summand of the module $\mathrm{S}_{a,\left\{\rho_{v}\right\},\left\{\chi_{v}\right\}}\left(\mathrm{U}_{1}(\mathrm{Q}), \mathscr{O}\right)$. Also Lemmas 3.1 .5 and 3.2 .2 of $[\mathrm{CHT}]$ tell us that

$$
\left(\prod_{v \in \mathbf{Q}} \mathrm{Q}_{\widetilde{v}}\left(\mathrm{~V}_{\varpi_{\tilde{v}}}\right)\right): \mathrm{H}_{\left\{\chi_{v}\right\}} \stackrel{\sim}{\longrightarrow} \mathrm{H}_{0,\left\{\chi_{v}\right\}, \mathrm{Q}} .
$$

Write $\mathbf{T}_{0,\left\{\chi_{v}\right\}, \mathcal{Q}}$ (resp. $\left.\mathbf{T}_{1,\left\{\chi_{v}\right\}, \mathrm{Q}}\right)$ for the image of $\mathbf{T}_{a,\left\{\rho_{v}\right\},\left\{\chi_{v}\right\}}^{\mathrm{T}(\mathrm{Q})}\left(\mathrm{U}_{1}(\mathrm{Q})\right.$ ) in the endomorphism ring of $\mathrm{H}_{0,\left\{\chi_{v}\right\}, Q}\left(\right.$ resp. $\left.\mathrm{H}_{1,\left\{\chi_{v}\right\}, \mathrm{Q}}\right)$. For all $v \in \mathrm{Q}, \mathrm{V}_{\varpi_{\tilde{v}}}=\mathrm{A}_{\widetilde{v}}$ on $\mathrm{H}_{1,\left\{\chi_{v}\right\}, \mathrm{Q}}$. By Part 8 of Proposition 3.4.4 of [CHT] we see that for each $v \in Q$ there is a character

$$
\mathrm{V}_{\widetilde{v}}: \mathrm{F}_{\widetilde{v}}^{\times} \longrightarrow \mathbf{T}_{1,\left\{\chi_{v}\right\}, Q}^{\times}
$$

such that

- if $\alpha \in \mathrm{F}_{\widetilde{v}}^{\times} \cap \mathscr{O}_{\mathrm{F}, \widetilde{v}}$ then $\mathrm{V}_{\widetilde{v}}(\alpha)=\mathrm{V}_{\alpha}$ on $\mathrm{H}_{1,\left\{\chi_{v}\right\}, Q}$, and

$-\left.\left(r_{\mathfrak{m}_{\left\{\chi_{v}\right\}, Q}} \otimes \mathbf{T}_{1,\left\{\chi_{v}\right\}, Q}\right)\right|_{\mathrm{W}_{\mathrm{F}_{\tilde{v}}}}=s \oplus\left(\mathrm{V}_{\widetilde{v}} \circ \operatorname{Art}_{\mathrm{F}_{\widetilde{v}}}^{-1}\right)$ where $s$ is unramified.

The representation $r_{\mathfrak{m}_{\left\{\chi_{v}\right\}, Q}}$ gives rise to a surjection

$$
\mathrm{R}_{\mathscr{S}_{\left\{x_{v}\right\}, \mathrm{Q}}}^{\mathrm{univ}} \rightarrow \mathbf{T}_{1,\left\{\chi_{v}\right\}, \mathrm{Q}}
$$

and the composite

$$
\prod_{v \in \mathrm{Q}} \mathscr{O}_{\mathrm{F}, \widetilde{v}}^{\times} \rightarrow \Delta_{\mathrm{Q}_{\mathrm{N}}} \longrightarrow\left(\mathrm{R}_{\mathscr{S}_{\left\{\chi_{v}\right\}, \mathrm{Q}}}^{\text {univ }}\right)^{\times} \longrightarrow \mathbf{T}_{1,\left\{\chi_{v}\right\}, \mathrm{Q}}^{\times}
$$

coincides with $\prod_{v} \mathrm{~V}_{\widetilde{v}}$. Thus $\mathrm{H}_{1,\left\{\chi_{v}\right\}, Q}$ becomes an $\mathrm{R}_{\mathscr{S}_{\left\{x_{v}\right\}, Q}^{\text {univ }}}^{\text {-module and we set }}$

$$
\mathrm{H}_{1,\left\{\chi_{v}\right\}, Q}^{\square_{\mathrm{T}}}=\mathrm{H}_{1,\left\{\chi_{v}\right\}, \mathrm{Q}} \otimes_{\mathrm{R}_{\mathscr{\{}\left\{x_{v}\right\}, Q}^{\text {univ }}} \mathrm{R}_{\mathscr{S}_{\left\{x_{v}\right\}, Q}}^{\square_{\mathrm{T}}}=\mathrm{H}_{1,\left\{\chi_{v}\right\}, \mathrm{Q}} \otimes_{\mathscr{O}} \mathscr{T} \text {. }
$$

As $\mathrm{H}_{1,\left\{\chi_{v}\right\}, Q}$ is a direct summand of $\mathrm{S}_{a,\left\{\rho_{v}\right\},\left\{\chi_{v}\right\}}\left(\mathrm{U}_{1}(\mathrm{Q}), \mathscr{O}\right)$ as modules for $\mathbf{T}_{a,\left\{\rho_{v}\right\},\left\{\chi_{v}\right\}}^{\mathrm{T}(\mathrm{Q})}\left(\mathrm{U}_{1}(\mathrm{Q})\right)$ and as $\operatorname{ker}\left(\prod_{v \in \mathrm{Q}} k(v)^{\times} \rightarrow \Delta_{\mathrm{Q}}\right)$ acts trivially on $\mathrm{H}_{1,\left\{\chi_{v}\right\}, \mathrm{Q}}$, Lemma 3.3.1 of [CHT] tells us that $\mathrm{H}_{1,\left\{\chi_{v}\right\}, \mathrm{Q}}$ is a free $\mathscr{O}\left[\Delta_{\mathrm{Q}}\right]$-module and that

$$
\left(\mathrm{H}_{1,\left\{\chi_{v}\right\}, Q}\right)_{\Delta_{\mathrm{Q}}} \stackrel{\sim}{\longrightarrow} \mathrm{H}_{0,\left\{\chi_{v}\right\}, \mathrm{Q}} \cong \mathrm{H}_{\left\{\chi_{v}\right\}} \text {. }
$$


As $\mathrm{U}$ is sufficiently small we get isomorphisms

$$
\begin{aligned}
\mathrm{S}_{a,\left\{\rho_{v}\right\},\left\{\chi_{v}\right\}}(\mathrm{U}, \mathscr{O}) \otimes_{\mathscr{O}} k & \cong \mathrm{S}_{a,\left\{\rho_{v}\right\},\left\{\chi_{v}\right\}}(\mathrm{U}, k)=\mathrm{S}_{a,\left\{\rho_{v}\right\},\{1\}}(\mathrm{U}, k) \\
& \cong \mathrm{S}_{a,\left\{\rho_{v}\right\},\{1\}}(\mathrm{U}, \mathscr{O}) \otimes_{\mathscr{O}} k
\end{aligned}
$$

and

$$
\begin{aligned}
\mathrm{S}_{a,\left\{\rho_{v}\right\},\left\{\chi_{v}\right\}}\left(\mathrm{U}_{1}(\mathrm{Q}), \mathscr{O}\right) \otimes_{\mathscr{O}} k & \cong \mathrm{S}_{a,\left\{\rho_{v}\right\},\left\{\chi_{v}\right\}}\left(\mathrm{U}_{1}(\mathrm{Q}), k\right)=\mathrm{S}_{a,\left\{\rho_{v}\right\},\{1\}}\left(\mathrm{U}_{1}(\mathrm{Q}), k\right) \\
& \cong \mathrm{S}_{a,\left\{\rho_{v}\right\},\{1\}}\left(\mathrm{U}_{1}(\mathrm{Q}), \mathscr{O}\right) \otimes_{\mathscr{O}} k .
\end{aligned}
$$

Thus we get identifications

$$
\mathrm{H}_{\left\{\chi_{v}\right\}} / \lambda \cong \mathrm{H}_{\{1\}} / \lambda
$$

and

$$
\mathrm{H}_{1,\left\{\chi_{v}\right\}, \mathrm{Q}} / \lambda \cong \mathrm{H}_{1,\{1\}, \mathrm{Q}} / \lambda
$$

compatible with the actions of $\mathrm{R}_{\mathscr{S}_{\left\{x_{v}\right\}, \emptyset}^{\text {univ }}}^{\text {ing }} / \lambda \cong \mathrm{R}_{\mathscr{S}_{\{1\}, \emptyset}^{\text {univ }}}^{\text {und }} / \lambda$ and of $\mathrm{R}_{\mathscr{S}_{\{x\}, Q}}^{\text {univ }} / \lambda \cong \mathrm{R}_{\mathscr{S}_{\{1\}, Q}}^{\text {univ }} / \lambda$, and also compatible with the maps $\mathrm{H}_{1,\left\{\chi_{v}\right\}, Q} / \lambda \rightarrow \mathrm{H}_{\left\{\chi_{v}\right\}} / \lambda$ and $\mathrm{H}_{1,\{1\}, \mathcal{Q}} / \lambda \rightarrow \mathrm{H}_{\{1\}} / \lambda$. Similarly we get an identification

$$
\mathrm{H}_{1,\left\{\chi_{v}\right\}, \mathrm{Q}}^{\square_{\mathrm{T}}} / \lambda \cong \mathrm{H}_{1,\{1\}, \mathrm{Q}}^{\square_{\mathrm{T}}} / \lambda
$$

compatible both with the isomorphism $\mathrm{R}_{\mathscr{S}_{\{x v\}, Q}}^{\square_{\mathrm{T}}} / \lambda \cong \mathrm{R}_{\mathscr{S}_{\{1\}, Q}}^{\square_{\mathrm{T}}} / \lambda$ and with the maps $\mathrm{H}_{1,\left\{\chi_{v}\right\}, \mathrm{Q}}^{\square_{\mathrm{T}}} / \lambda \rightarrow \mathrm{H}_{\left\{\chi_{v}\right\}} / \lambda$ and $\mathrm{H}_{1,\{1\}, \mathrm{Q}}^{\square_{\mathrm{T}}} / \lambda \rightarrow \mathrm{H}_{\{1\}} / \lambda$.

Proposition 1.5 .9 of $[\mathrm{CHT}]$ tells us that there is a non-negative integer $q$, such that for any positive integer $\mathrm{N}$ there is a set $\mathrm{Q}_{\mathrm{N}}$ (and $\bar{\psi}_{w}$ for $w \mid v \in \mathrm{Q}_{\mathrm{N}}$ ) as above such that

$-\# \mathrm{Q}_{\mathrm{N}}=q \geq\left[\mathrm{F}^{+}: \mathbf{Q}\right] n(n-1) / 2+\left[\mathrm{F}^{+}: \mathbf{Q}\right] n\left(1-(-1)^{\mu_{\mathfrak{m}}-n}\right) / 2$;

- if $v \in Q_{\mathrm{N}}$ then $\mathbf{N} v \equiv 1 \bmod l^{\mathrm{N}}$; and

- $\mathrm{R}_{\mathscr{S}_{\{1\}, Q_{N}}^{\square_{\mathrm{T}}}}$ can be topologically generated over $\mathrm{R}_{\{1\}, \mathrm{T}}^{\text {loc }}$ by

$$
q-\left[\mathrm{F}^{+}: \mathbf{Q}\right] n(n-1) / 2-\left[\mathrm{F}^{+}: \mathbf{Q}\right] n\left(1-(-1)^{\mu_{\mathfrak{m}}-n}\right) / 2
$$

elements.

Set

$$
\begin{aligned}
& -q^{\prime}=q-\left[\mathrm{F}^{+}: \mathbf{Q}\right] n(n-1) / 2-\left[\mathrm{F}^{+}: \mathbf{Q}\right] n\left(1-(-1)^{\mu_{\mathfrak{m}}-n}\right) / 2 \geq 0 \\
& - \text { and } \mathrm{R}_{\left\{\chi_{v}\right\}, \infty}^{\square_{\mathrm{T}}}=\mathrm{R}_{\left\{\chi_{v}\right\}, \mathrm{T}}^{\operatorname{loc}}\left[\left[\mathrm{Y}_{1}, \ldots, \mathrm{Y}_{q^{\prime}}\right]\right] .
\end{aligned}
$$


Suppose that either $\chi_{v}=1$ for all $v \in \mathrm{R}$ or that $\chi_{v, i} \neq \chi_{v, j}$ for all $v \in \mathrm{R}$ and all $i \neq j$. Then all irreducible components of $\operatorname{Spec} \mathrm{R}_{\left\{\chi_{v}\right\}, \infty}^{\square_{\mathrm{T}}}$ have dimension

$$
1+q+n^{2} \# \mathrm{~T}-\left[\mathrm{F}^{+}: \mathbf{Q}\right] n\left(1-(-1)^{\mu_{\mathfrak{m}}-n}\right) / 2
$$

and their generic points have characteristic 0 . If for all $v \in \mathrm{R}$ the characters $\chi_{v, i}$ are all distinct for $i=1, \ldots, n$ then $\operatorname{Spec} \mathrm{R}_{\left\{\chi_{v}\right\}, \infty}^{\square_{\mathrm{T}}}$ is irreducible. Every prime of $\mathrm{R}_{\{1\}, \infty}^{\square_{\mathrm{T}}}$, which is minimal over $\lambda \mathrm{R}_{\{1\}, \infty}^{\square_{\mathrm{T}}}$, contains a unique minimal prime of $\mathrm{R}_{\{1\}, \infty}^{\square_{\mathrm{T}}}$. (See Proposition 3.1 and Section 2.4 of [CHT].)

Note that we have a natural identification

$$
\mathrm{R}_{\left\{\chi_{v}\right\}, \infty}^{\square_{\mathrm{T}}} / \lambda \cong \mathrm{R}_{\{1\}, \infty}^{\square_{\mathrm{T}}} / \lambda \text {. }
$$

Choose a surjection

$$
\mathrm{R}_{\{1\}, \infty}^{\square_{\mathrm{T}}} \rightarrow \mathrm{R}_{\mathscr{S}_{\{1\}, Q_{\mathrm{N}}}^{\square_{\mathrm{T}}}}
$$

extending the natural map $\mathrm{R}_{\{1\}, \mathrm{T}}^{\text {loc }} \rightarrow \mathrm{R}_{\mathscr{S}_{\{1\}, Q_{\mathrm{N}}}}^{\square_{\mathrm{T}}}$. Reducing modulo $\lambda$ and applying the natural comparisons, this gives rise to a surjection

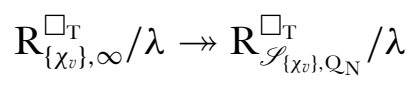

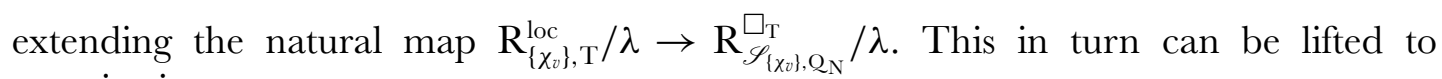
a surjection

$$
\mathrm{R}_{\left\{\chi_{v}\right\}, \infty}^{\square_{\mathrm{T}}} \rightarrow \mathrm{R}_{\mathscr{S}_{\left\{x_{i}\right\}, \mathrm{Q}_{\mathrm{N}}}^{\square_{\mathrm{T}}}}
$$

extending the natural map $\mathrm{R}_{\left\{\chi_{v}\right\}, \mathrm{T}}^{\text {loc }} \rightarrow \mathrm{R}_{\mathscr{S}_{\left\{x_{v}\right\}, \mathrm{Q}_{\mathrm{N}}}^{\square_{\mathrm{T}}}}$.

Also set

$-\Delta_{\infty}=\mathbf{Z}_{l}^{q}$

$-\mathrm{S}_{\infty}=\mathscr{T}\left[\left[\Delta_{\infty}\right]\right]$

- and $\mathfrak{a}=\operatorname{ker}\left(\mathrm{S}_{\infty} \rightarrow \mathscr{O}\right)$, where the map sends each element of $\Delta_{\infty}$ to 1 and each $\mathrm{X}_{v, i, j}$ to 0 .

Thus $\mathrm{S}_{\infty}$ is isomorphic to a power series ring in $q+n^{2} \# \mathrm{~T}$ variables over $\mathscr{O}$ in such a way that $\mathfrak{a}$ is the ideal generated by these variables. Choose continuous surjections

$$
\Delta_{\infty} \rightarrow \Delta_{Q_{N}}
$$

for all $\mathrm{N}$ and let $\mathfrak{c}_{\mathrm{N}}$ denote the kernel of the corresponding map

$$
\mathscr{O}\left[\left[\Delta_{\infty}\right]\right] \rightarrow \mathscr{O}\left[\Delta_{\mathrm{Q}_{\mathrm{N}}}\right] .
$$

Note that any open ideal of $\mathscr{O}\left[\left[\Delta_{\infty}\right]\right]$ contains $\mathfrak{c}_{\mathrm{N}}$ for all sufficiently large $\mathrm{N}$. The choice of $\Delta_{\infty} \rightarrow \Delta_{Q_{N}}$ gives rise to a map

$$
\mathrm{S}_{\infty} \rightarrow \mathscr{T}\left[\Delta_{\mathrm{Q}_{N}}\right] \longrightarrow \mathrm{R}_{\mathscr{S}_{\left\{x_{v}\right\}, \mathrm{Q}_{\mathrm{N}}}^{\square_{\mathrm{T}}}} \text {. }
$$


We see that

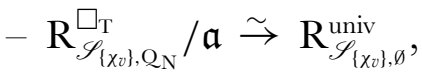

$-\mathrm{H}_{1,\left\{\chi_{\imath}\right\}, \mathrm{Q}_{\mathrm{N}}}^{\square_{\mathrm{T}}}$ is finite free over $\mathrm{S}_{\infty} / \mathfrak{c}_{\mathrm{N}}$,

$-\mathrm{H}_{1,\left\{\chi_{v}\right\}, Q_{N}}^{\square_{\mathrm{T}}} / \mathfrak{a} \stackrel{\sim}{\rightarrow} \mathrm{H}_{\left\{\chi_{v}\right\}}$.

Our aim is to 'patch' the $\mathrm{R}_{\mathscr{S}_{\left\{\chi_{v}\right\}, Q_{\mathrm{N}}}^{\square_{\mathrm{T}}}}$ to form in the limit a copy of $\mathrm{R}_{\left\{\chi_{v}\right\}, \infty}^{\square_{\mathrm{T}}}$ and simultaneously patch the $\mathrm{H}_{1,\left\{\chi_{v}\right\}, \mathrm{Q}_{\mathrm{N}}}^{\square_{\mathrm{T}}}$ to produce a $\mathrm{R}_{\left\{\chi_{v}\right\}, \infty}^{\square_{\mathrm{T}}}$-module, which is also finite and free over $\mathrm{S}_{\infty}$. To do this patching it is better to work with finite cardinality

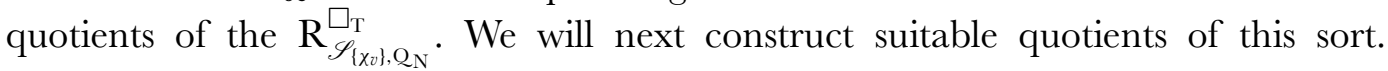

Choose open ideals $\mathfrak{b}_{\mathrm{N}}$ in $\mathrm{S}_{\infty}$ such that

$-\mathfrak{b}_{\mathrm{N}} \supset \mathfrak{a}_{\mathrm{N}}$

$-\mathfrak{b}_{\mathrm{N}} \supset \mathfrak{b}_{\mathrm{N}+1}$,

- and $\bigcap_{\mathrm{N}} \mathfrak{b}_{\mathrm{N}}=(0)$.

Also choose open ideals $\mathfrak{d}_{\left\{\chi_{v}\right\}, \mathrm{N}}$ in $\mathrm{R}_{\mathscr{S}_{\left\{x_{v}\right\}, \emptyset}^{\text {univ }}}$ such that

$-\operatorname{ker}\left(\mathrm{R}_{\mathscr{S}_{\left\{x_{v}\right\}, \emptyset}^{\text {univ }}} \rightarrow \mathbf{T}_{\left\{\chi_{v}\right\}}\right)+\mathfrak{b}_{\mathrm{N}} \mathrm{R}_{\mathscr{S}_{\left\{x_{x}\right\}, \emptyset}}^{\text {univ }} \supset \mathfrak{d}_{\left\{\chi_{v}\right\}, \mathrm{N}} \supset \mathfrak{b}_{\mathrm{N}} \mathrm{R}_{\mathscr{S}_{\left\{x_{v}\right\}, \emptyset}^{\text {univ }}}^{\text {un }}$

$-\mathfrak{d}_{\left\{\chi_{v}\right\}, \mathrm{N}} \supset \mathfrak{d}_{\left\{\chi_{v}\right\}, \mathrm{N}+1}$;

$-\bigcap_{\mathrm{N}} \mathfrak{d}_{\left\{\chi_{v}\right\}, \mathrm{N}}=(0)$;

$-\left(\mathfrak{d}_{\left\{\chi_{v}\right\}, \mathrm{N}} \bmod \lambda\right)=\left(\mathfrak{d}_{\{1\}, \mathrm{N}} \bmod \lambda\right)$ in $\mathrm{R}_{\mathscr{S}_{\left\{\chi_{v}\right\}, \emptyset}^{\text {univ }}} / \lambda=\mathrm{R}_{\mathscr{S}_{\{1\}, \mathscr{Q}}}^{\text {univ }} / \lambda$.

(Set

$$
\mathfrak{d}_{\left\{\chi_{v}\right\}, \mathrm{N}}^{\prime}=\left(\operatorname{ker}\left(\mathrm{R}_{\mathscr{S}_{\left\{x_{v}\right\}, \emptyset}^{\text {univ }}}^{\prime} \rightarrow \mathbf{T}_{\left\{\chi_{v}\right\}}\right)+\mathfrak{b}_{\mathrm{N}} \mathrm{R}_{\mathscr{S}_{\left\{x_{x}\right\}, \emptyset}^{\text {univ }}}^{\text {und }}\right) \cap\left(\mathfrak{m}_{\mathrm{R}_{\left\{x_{v}\right\}, \emptyset}^{\mathrm{univ}}}^{\mathrm{N}}+\mathfrak{b}_{\mathrm{N}} \mathrm{R}_{\left\{\chi_{v}\right\}, \emptyset}^{\text {univ }}\right)
$$

As $\mathbf{T}_{\{1\}}$ is finite over $\mathscr{O}$, the ideal $\mathfrak{b}_{\mathrm{N}} \mathbf{T}_{\{1\}}$ is open in $\mathbf{T}_{\{1\}}$ and so $\mathfrak{d}_{\left\{\chi_{v}\right\}, \mathrm{N}}^{\prime}$ will be

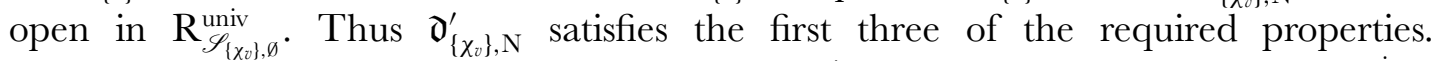
Then define $\mathfrak{d}_{\left\{\chi_{v}\right\}, \mathrm{N}}$ to be the intersection of $\mathfrak{d}_{\left\{\chi_{v}\right\}, \mathrm{N}}^{\prime}$ and the preimages in $\mathrm{R}_{\mathscr{S}_{\left\{x_{v}\right\}, \emptyset}^{\text {univ }}}^{\text {. }}$ of $\mathfrak{d}_{\left\{\chi_{v}^{\prime}\right\}, N}^{\prime} \bmod \lambda$ in $\mathrm{R}_{\mathscr{S}_{\left\{\chi_{v}^{\prime}\right\}, \emptyset}}^{\text {univ }} / \lambda=\mathrm{R}_{\mathscr{S}_{\left\{x_{0}\right\}, \emptyset}}^{\text {univ }} / \lambda$ for all (the finite number of) $\left\{\chi_{v}^{\prime}\right\}$.)

Thus we obtain maps

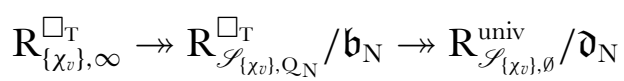

which agree modulo $\lambda$. If $\mathrm{M} \geq \mathrm{N}$ then

$$
\mathrm{H}_{1,\left\{\chi_{v}\right\}, \mathrm{Q}_{\mathrm{M}}}^{\square_{\mathrm{T}}} / \mathfrak{b}_{\mathrm{N}}
$$

becomes an $\mathrm{R}_{\left\{\chi_{v}\right\}, \infty}^{\square_{\mathrm{T}}} \widehat{\otimes}_{\mathscr{O}} \mathrm{S}_{\infty} / \mathfrak{b}_{\mathrm{N}}$-module which is finite free over $\mathrm{S}_{\infty} / \mathfrak{b}_{\mathrm{N}}$ and such that the image of $\mathrm{S}_{\infty}$ in $\operatorname{End}\left(\mathrm{H}_{1,\left\{\chi_{v}\right\}, \mathrm{Q}_{\mathrm{M}}}^{\square_{\mathrm{T}}} / \mathfrak{b}_{\mathrm{N}}\right)$ is contained in the image of $\mathrm{R}_{\left\{\chi_{v}\right\}, \infty}^{\square_{\mathrm{T}}}$. Moreover

$$
\mathrm{H}_{1,\left\{\chi_{v}\right\}, \mathrm{Q}_{\mathrm{M}}}^{\square_{\mathrm{T}}} /\left(\mathfrak{b}_{\mathrm{N}}+\lambda\right) \cong\left(\mathrm{H}_{1,\{1\}, \mathrm{Q}_{\mathrm{M}}}^{\square_{\mathrm{T}}}\right) /\left(\mathfrak{b}_{\mathrm{N}}+\lambda\right)
$$


as $\mathrm{R}_{\left\{\chi_{v}\right\}, \infty}^{\square_{\mathrm{T}}} / \lambda=\left(\mathrm{R}_{\{1\}, \infty}^{\square_{\mathrm{T}}}\right) / \lambda$-modules. Finally we have isomorphisms of $\mathbf{R}_{\left\{\chi_{v}\right\}, \infty}^{\square_{\mathrm{T}}}$-modules

$$
\mathrm{H}_{1,\left\{\chi_{v}\right\}, \mathrm{Q}_{\mathrm{M}}}^{\square_{\mathrm{T}}} /\left(\mathfrak{b}_{\mathrm{N}}+\mathfrak{a}\right) \stackrel{\sim}{\longrightarrow} \mathrm{H}_{\left\{\chi_{v}\right\}} / \mathfrak{b}_{\mathrm{N}}
$$

which are compatible modulo $\lambda .\left(\mathbf{R}_{\left\{\chi_{v}\right\}, \infty}^{\square_{\mathrm{T}}}\right.$ acts on the right hand module via the $\operatorname{map} \mathrm{R}_{\left\{\chi_{v}\right\}, \infty}^{\square_{\mathrm{T}}} \rightarrow \mathrm{R}_{\mathscr{S}_{\left\{\chi_{v}\right\}, \emptyset}^{\text {univ }}}^{\text {mat }} / \mathfrak{d}_{\mathrm{N}}$.

Because $\mathrm{R}_{\left\{\chi_{v}\right\}, \infty}^{\square_{\mathrm{T}}, \infty} \widehat{\otimes}_{\mathscr{O}} \mathrm{S}_{\infty}$ is topologically finitely generated and because the module $\mathrm{H}_{1,\left\{\chi_{v}\right\}, Q_{\mathrm{M}}}^{\square_{\mathrm{T}}} / \mathfrak{b}_{\mathrm{N}}$ and the ring $\mathrm{R}_{\mathscr{S}_{\left\{\chi_{i}\right\}, \emptyset}^{\text {univ }}} / \mathfrak{d}_{\mathrm{N}}$ have finite cardinality independent of $\mathrm{M} \geq \mathrm{N}$ we can find an infinite sequence of pairs of integers $\left(\mathrm{M}_{i}, \mathrm{~N}_{i}\right)$ with $\mathrm{M}_{i} \geq \mathrm{N}_{i}$ such that

$-\mathrm{N}_{i+1}>\mathrm{N}_{i}$ and $\mathrm{M}_{i+1}>\mathrm{M}_{i}$

- the reduction modulo $\mathfrak{d}_{\mathrm{N}_{i}}$ of the maps $\mathrm{R}_{\left\{\chi_{v}\right\}, \infty}^{\square_{\mathrm{T}}} \rightarrow \mathrm{R}_{\mathscr{S}_{\left\{\chi_{v}\right\}, \emptyset}^{\text {univ }}} / \mathfrak{d}_{\mathrm{N}_{i+1}}$ equals the map $\mathrm{R}_{\left\{\chi_{v}\right\}, \infty}^{\square_{\mathrm{T}}} \rightarrow \mathrm{R}_{\mathscr{S}_{\left\{x_{v}\right\}, \emptyset}^{\text {univ }}}^{\mathrm{u}_{1}} / \mathfrak{d}_{\mathrm{N}_{i}}$ for all (the finite number of choices for) $\left\{\chi_{v}\right\}$;

- there are isomorphisms of $\mathrm{R}_{\left\{\chi_{v}\right\}, \infty}^{\square_{\mathrm{T}}} \widehat{\otimes}_{\mathscr{O}} \mathrm{S}_{\infty}$-modules

$$
\mathrm{H}_{1,\left\{\chi_{v}\right\}, \mathrm{Q}_{\mathrm{M}_{i+1}}^{\square}}^{\square_{\mathrm{T}}} / \mathfrak{b}_{\mathrm{N}_{i}} \stackrel{\sim}{\longrightarrow} \mathrm{H}_{1,\left\{\chi_{v}\right\}, \mathrm{Q}_{\mathrm{M}}}^{\square_{\mathrm{T}}} / \mathfrak{b}_{\mathrm{N}_{i}}
$$

compatible both with the isomorphisms

$$
\mathrm{H}_{1,\left\{\chi_{v}\right\}, \mathrm{QM}_{i+1}}^{\square_{\mathrm{T}}} /\left(\lambda+\mathfrak{b}_{\mathrm{N}_{i}}\right) \cong\left(\mathrm{H}_{1,\{1\}, \mathrm{QM}_{i+1}}^{\square_{\mathrm{T}}}\right) /\left(\lambda+\mathfrak{b}_{\mathrm{N}_{i}}\right)
$$

and

$$
\mathrm{H}_{1,\left\{\chi_{i}\right\}, Q_{\mathrm{M}_{i}}}^{\square_{\mathrm{T}}} /\left(\lambda+\mathfrak{b}_{\mathrm{N}_{i}}\right) \cong\left(\mathrm{H}_{1,\{1\}, \mathrm{Q}_{\mathrm{M}_{i}}}^{\square_{\mathrm{T}}}\right) /\left(\lambda+\mathfrak{b}_{\mathrm{N}_{i}}\right) ;
$$

and with the maps

$$
\mathrm{H}_{1,\left\{\chi_{v}\right\}, Q_{\mathrm{M}_{i+1}}}^{\square} / \mathfrak{b}_{\mathrm{N}_{i}} \rightarrow \mathrm{H}_{\left\{\chi_{v}\right\}} / \mathfrak{b}_{\mathrm{N}_{i}}
$$

and

$$
\mathrm{H}_{1,\left\{\chi_{v}\right\}, Q_{\mathrm{M}_{i}}}^{\square} / \mathfrak{b}_{\mathrm{N}_{i}} \rightarrow \mathrm{H}_{\left\{\chi_{v}\right\}} / \mathfrak{b}_{\mathrm{N}_{i}} .
$$

Taking the limit as $i \rightarrow \infty$ we get maps

$$
\mathrm{R}_{\left\{\chi_{v}\right\}, \infty}^{\square_{\mathrm{T}}} \rightarrow \mathrm{R}_{\mathscr{S}_{\left\{\chi_{v}\right\}, \emptyset}}^{\text {univ }}
$$

which agree modulo $\lambda$.

Set

$$
\mathrm{H}_{1,\left\{\chi_{v}\right\}, \infty}^{\square_{\mathrm{T}}}=\lim _{\leftarrow} \mathrm{H}_{1,\left\{\chi_{v}\right\}, \mathrm{QM}_{i}}^{\square_{\mathrm{T}}} / \mathfrak{b}_{\mathrm{N}_{i}} .
$$


Thus $\mathrm{H}_{1,\left\{\chi_{v}\right\}, \infty}^{\square \mathrm{T}}$ is an $\mathrm{R}_{\left\{\chi_{v}\right\}, \infty}^{\square_{\mathrm{T}}} \widehat{\otimes}_{\mathscr{O}} \mathrm{S}_{\infty}$-module. The action of $\mathrm{S}_{\infty}$ can be factored through $\mathrm{R}_{\left\{\chi_{v}\right\}, \infty}^{\square_{\mathrm{T}}}$. (As $\mathrm{S}_{\infty}$ is formally smooth.) The lifts

$$
\mathrm{S}_{\infty} \rightarrow \mathrm{R}_{\left\{\chi_{v}\right\}, \infty}^{\square_{\mathrm{T}}}
$$

can be chosen to agree modulo $\lambda$. There is a natural isomorphism

$$
\mathrm{H}_{1,\left\{\chi_{v}\right\}, \infty}^{\square_{\mathrm{T}}} / \lambda \cong \mathrm{H}_{1,\{1\}, \infty}^{\square_{\mathrm{T}}} / \lambda
$$

of $\mathrm{R}_{\left\{\chi_{\vartheta}\right\}, \infty}^{\square_{\mathrm{T}}} / \lambda \widehat{\otimes}_{\mathscr{O}} \mathrm{S}_{\infty} \cong \mathrm{R}_{\{1\}, \infty}^{\square} / \lambda \widehat{\otimes}_{\mathscr{O}} \mathrm{S}_{\infty}$-modules. There are also isomorphisms

$$
\mathrm{H}_{1,\left\{\chi_{v}\right\}, \infty}^{\square} / \mathfrak{a} \stackrel{\sim}{\longrightarrow} \mathrm{H}_{\left\{\chi_{v}\right\}}
$$

which agree modulo $\lambda$ and are compatible with the maps

$$
\mathrm{R}_{\left\{\chi_{v}\right\}, \infty}^{\square \mathrm{T}} \rightarrow \mathrm{R}_{\mathscr{S}_{\left\{\chi_{v}\right\}, \emptyset}^{\text {univ }}}^{\square} \rightarrow \mathbf{T}_{\left\{\chi_{v}\right\}} \text {. }
$$

Now choose characters $\chi_{v}$ for $v \in \mathrm{R}$ such that $\chi_{v, i} \neq \chi_{v, j}$ for $i \neq j$. (This is possible as $l>n$.) As $\mathrm{H}_{\left\{\chi_{v}\right\}, \infty}^{\square_{\mathrm{T}}}$ is finite, free over $\mathrm{S}_{\infty}$ and $\mathrm{H}_{\left\{\chi_{v}\right\}, \infty}^{\square_{\mathrm{T}}} / \mathfrak{a} \cong \mathrm{H}_{\left\{\chi_{v}\right\}}$ we see that $\mathrm{H}_{\left\{\chi_{v}\right\}, \infty}^{\square_{\mathrm{T}}}$ has $\mathrm{R}_{\left\{\chi_{v}\right\}, \infty}^{\square_{\mathrm{T}}}$-depth at least $1+q+n^{2} \# \mathrm{~T}$. Thus $\mathrm{R}_{\left\{\chi_{v}\right\}, \infty}^{\square_{\mathrm{T}}} / \operatorname{Ann} \mathrm{H}_{1,\left\{\chi_{v}\right\}, \infty}^{\square_{\mathrm{T}}}$ has dimension at least $1+q+n^{2} \# \mathrm{~T}$. However $\mathrm{R}_{\left\{\chi_{v}\right\}, \infty}^{\square_{\mathrm{T}}}$ has dimension

$$
1+q+n^{2} \# \mathrm{~T}-\left[\mathrm{F}^{+}: \mathbf{Q}\right] n\left(1-(-1)^{\mu_{\mathfrak{m}}-n}\right) / 2
$$

and a unique minimal ideal. Thus (see Lemma 2.3) $\mu_{m} \equiv n \bmod 2$ and $\mathrm{H}_{\left\{\chi_{v}\right\}, \infty}^{\square_{\mathrm{T}}}$ is a nearly faithful $\mathrm{R}_{\left\{\chi_{v}\right\}, \infty}^{\square_{\mathrm{T}}}$-module.

We also deduce that $\mathrm{H}_{1,\left\{\chi_{v}\right\}, \infty}^{\square_{\mathrm{T}}} / \lambda \cong \mathrm{H}_{1,\{1\}, \infty}^{\square_{\mathrm{T}}} / \lambda$ is a nearly faithful as a module over $\mathrm{R}_{\left\{\chi_{v}\right\}, \infty}^{\square_{\mathrm{T}}} / \lambda \cong \mathrm{R}_{\{1\}, \infty}^{\square_{\mathrm{T}}} / \lambda$. (See Section 2 for the definition of 'nearly faithful'.) Recall that all generic points of $\operatorname{Spec} \mathrm{R}_{\{1\}, \infty}^{\square_{\mathrm{T}}}$ have characteristic zero and every prime of $R_{\{1\}, \infty}^{\square_{T}}$, which is minimal over $\lambda R_{\{1\}, \infty}^{\square_{T}}$, contains a unique minimal prime of $\mathrm{R}_{\{1\}, \infty}^{\square_{\mathrm{T}}}$. Thus Lemma 2.2 implies that $\mathrm{H}_{1,\{1\}, \infty}^{\square_{\mathrm{T}}}$ is a nearly faithful $\mathrm{R}_{\{1\}, \infty}^{\square_{\mathrm{T}}}$-module. Finally this implies that $\mathrm{H}$ is a nearly faithful $\mathrm{R}_{\mathscr{S}}^{\text {univ }}$-module. As $\mathbf{T}$ is reduced, the theorem follows.

\section{Modularity lifting theorems}

In this section we combine Theorem 4.1 with the theory of base change to deduce more explicit and useful modularity lifting theorems.

Let $\mathrm{F}$ be a CM field. By a RACSDC (regular, algebraic, conjugate self dual, cuspidal) automorphic representation $\pi$ of $\mathrm{GL}_{n}\left(\mathbf{A}_{\mathrm{F}}\right)$ we mean a cuspidal automorphic representation such that 
$-\pi^{\vee} \cong \pi^{c}$, and

$-\pi_{\infty}$ has the same infinitesimal character as some irreducible algebraic representation of the restriction of scalars from $\mathbf{F}$ to $\mathbf{Q}$ of $\mathrm{GL}_{n}$.

Let $a \in\left(\mathbf{Z}^{n}\right)^{\operatorname{Hom}(\mathrm{F}, \mathbf{C})}$ satisfy

$-a_{\tau, 1} \geq \ldots \geq a_{\tau, n}$, and

$-a_{\tau c, i}=-a_{\tau, n+1-i}$.

Let $\boldsymbol{\Xi}_{a}$ denote the irreducible algebraic representation of $\mathrm{GL}_{n}^{\mathrm{Hom}(\mathrm{F}, \mathbf{C})}$ which is the tensor product over $\tau$ of the irreducible representations of $\mathrm{GL}_{n}$ with highest weights $a_{\tau}$. We will say that a RACSDG automorphic representation $\pi$ of $\mathrm{GL}_{n}\left(\mathbf{A}_{\mathrm{F}}\right)$ has weight $a$ if $\pi_{\infty}$ has the same infinitessimal character as $\boldsymbol{\Xi}_{a}^{\vee}$.

Let $\mathrm{S}$ be a finite set of finite places of F. For $v \in \mathrm{S}$ let $\rho_{v}$ be an irreducible square integrable representation of $\mathrm{GL}_{n}\left(\mathrm{~F}_{v}\right)$. We will say that a RACSDC automorphic representation $\pi$ of $\mathrm{GL}_{n}\left(\mathbf{A}_{\mathrm{F}}\right)$ has type $\left\{\rho_{v}\right\}_{v \in \mathrm{S}}$ if for each $v \in \mathrm{S}, \pi_{v}$ is an unramified twist of $\rho_{v}^{\vee}$.

The following is a restatement of Theorem VII.1.9 of [HT].

Proposition 5.1. - Let $\boldsymbol{\imath}: \overline{\mathbf{Q}}_{l} \stackrel{\sim}{\rightarrow} \mathbf{C}$. Let $\mathrm{F}$ be an imaginary $C M$ field, $\mathrm{S}$ a finite non-empty set of finite places of $\mathrm{F}$ and, for $v \in \mathrm{S}, \rho_{v}$ a square integrable representation of $\mathrm{GL}_{n}\left(\mathrm{~F}_{v}\right)$. Let $a \in\left(\mathbf{Z}^{n}\right)^{\mathrm{Hom}(\mathrm{F}, \mathbf{C})}$ be as above. Suppose that $\pi$ is a RACSDC automorphic representation of $\mathrm{GL}_{n}\left(\mathbf{A}_{\mathrm{F}}\right)$ of weight $a$ and type $\left\{\rho_{v}\right\}_{v \in \mathrm{S}}$. Then there is a continuous semisimple representation

$$
r_{l, l}(\pi): \operatorname{Gal}(\overline{\mathrm{F}} / \mathrm{F}) \longrightarrow \mathrm{GL}_{n}\left(\overline{\mathbf{Q}}_{l}\right)
$$

with the following properties.

1. For every prime ołl of $\mathrm{F}$ we have

$$
\left.r_{l, l}(\pi)\right|_{\mathrm{Gal}\left(\overline{\mathrm{F}}_{v} / \mathrm{F}_{v}\right)} ^{\mathrm{ss}}=r_{l}\left(l^{-1} \pi_{v}\right)^{\vee}(1-n)^{\mathrm{ss}} .
$$

2. $r_{l, l}(\pi)^{c}=r_{l, l}(\pi)^{\vee} \epsilon^{1-n}$.

3. If $v \mid l$ is a prime of $\mathrm{F}$ then $\left.r_{l, l}(\pi)\right|_{\mathrm{Gal}\left(\overline{\mathrm{F}}_{v} / \mathrm{F}_{v}\right)}$ is potentially semistable, and if $\pi_{v}$ is unramified then it is crystalline.

4. If $v \mid l$ is a prime of $\mathrm{F}$ and if $\tau: \mathrm{F} \hookrightarrow \overline{\mathbf{Q}}_{l}$ lies above $v$ then

$$
\operatorname{dim}_{\overline{\mathbf{Q}}_{l}} \operatorname{gr}^{i}\left(r_{l, l}(\pi) \otimes_{\tau, \mathrm{F}_{v}} \mathrm{~B}_{\mathrm{DR}}\right)^{\mathrm{Gal}\left(\overline{\mathrm{F}}_{v} / \mathrm{F}_{v}\right)}=0
$$

unless $i=a_{\imath \tau, j}+n-j$ for some $j=1, \ldots, n$ in which case

$$
\operatorname{dim}_{\overline{\mathbf{Q}} l} \operatorname{gr}^{i}\left(r_{l, l}(\pi) \otimes_{\tau, \mathrm{F}_{v}} \mathrm{~B}_{\mathrm{DR}}\right)^{\mathrm{Gal}\left(\overline{\mathrm{F}}_{v} / \mathrm{F}_{v}\right)}=1 .
$$

Proof. - We can take $r_{l, l}(\pi)=\mathrm{R}_{l}\left(\pi^{\vee}\right)(1-n)$ in the notation of [HT]. Note that the definition of highest weight we use here differs from that in [HT]. 
The representation $r_{l, l}(\pi)$ can be taken to be valued in $\operatorname{GL}_{n}(\mathscr{O})$ where $\mathscr{O}$ is the ring of integers of some finite extension of $\mathbf{Q}_{l}$. Thus we can reduce it modulo the maximal ideal of $\mathscr{O}$ and semisimplify to obtain a continuous semisimple representation

$$
\bar{r}_{l, l}(\pi): \operatorname{Gal}(\overline{\mathrm{F}} / \mathrm{F}) \longrightarrow \mathrm{GL}_{n}\left(\overline{\mathbf{F}}_{l}\right)
$$

which is independent of the choices made.

Let $l: \overline{\mathbf{Q}}_{l} \stackrel{\sim}{\rightarrow}$ C. Suppose that $a \in\left(\mathbf{Z}^{n}\right)^{\operatorname{Hom}\left(\mathrm{F}, \overline{\mathbf{Q}}_{l}\right)}$ satisfies

$-a_{\tau, 1} \geq \ldots \geq a_{\tau, n}$, and

$-a_{\tau c, i}=-a_{\tau, n+1-i}$.

Then we define $l_{*} a$ by

$$
\left(l_{*} a\right)_{\imath \tau, i}=a_{\tau, i} .
$$

Suppose also that $\mathrm{S}$ is a finite set of finite places of $\mathrm{F}$ not containing any prime above $l$ and that $\rho_{v}$ is a discrete series representation of $\mathrm{GL}_{n}\left(\mathrm{~F}_{v}\right)$ over $\overline{\mathbf{Q}}_{l}$ for all $v \in \mathrm{S}$. We will call a continuous semisimple representation

$$
r: \operatorname{Gal}(\overline{\mathrm{F}} / \mathrm{F}) \longrightarrow \mathrm{GL}_{n}\left(\overline{\mathbf{Q}}_{l}\right)
$$

(resp.

$$
\left.\bar{r}: \operatorname{Gal}(\overline{\mathrm{F}} / \mathrm{F}) \longrightarrow \mathrm{GL}_{n}\left(\overline{\mathbf{F}}_{l}\right)\right)
$$

automorphic of weight $a$ and type $\left\{\rho_{v}\right\}_{v \in \mathrm{S}}$ if there is an isomorphism $l: \overline{\mathbf{Q}}_{l} \stackrel{\sim}{\rightarrow} \mathbf{C}$ and a RACSDC automorphic representation $\pi$ of $\mathrm{GL}_{n}\left(\mathbf{A}_{\mathrm{F}}\right)$ of weight $\boldsymbol{l}_{*} a$ and type $\left\{\imath \rho_{v}\right\}_{v \in \mathrm{S}}$ (resp. and with $\pi_{l}$ unramified) such that $r \cong r_{l, l}(\pi)$ (resp. $\left.\bar{r} \cong \bar{r}_{l, l}(\pi)\right)$. We will say that $r$ is automorphic of weight $a$ and type $\left\{\rho_{v}\right\}_{v \in \mathrm{S}}$ and level prime to $l$ if there is an isomorphism $l: \overline{\mathbf{Q}}_{l} \stackrel{\sim}{\rightarrow} \mathbf{C}$ and a RACSDG automorphic representation $\pi$ of $\mathrm{GL}_{n}\left(\mathbf{A}_{\mathrm{F}}\right)$ of weight $l_{*} a$ and type $\left\{l \rho_{v}\right\}_{v \in \mathrm{S}}$ and with $\pi_{l}$ unramified such that $r \cong r_{l, l}(\pi)$.

Recall that (see Definition 2.5.1 of $[\mathrm{CHT}]$ ) we call a subgroup $\mathrm{H} \subset \mathrm{GL}_{n}(k)$ big if the following conditions are satisfied.

- H has no $l$-power order quotient.

$-\mathrm{H}^{i}\left(\mathrm{H}, \mathfrak{g l}_{n}^{0}(k)\right)=(0)$ for $i=0,1$.

- For all irreducible $k[\mathrm{H}]$-submodules $\mathrm{W}$ of $\mathfrak{g l}_{n}^{0}(k)$ we can find $h \in \mathrm{H}$ and $\alpha \in k$ with the following properties. The $\alpha$ generalised eigenspace $\mathrm{V}_{h, \alpha}$ of $h$ in $k^{n}$ is one dimensional. Let $\pi_{h, \alpha}: k^{n} \rightarrow \mathrm{V}_{h, \alpha}$ (resp. $i_{h, \alpha}$ ) denote the $h$-equivariant projection of $k^{n}$ to $\mathrm{V}_{h, \alpha}$ (resp. h-equivariant injection of $\mathrm{V}_{h, \alpha}$ into $k^{n}$. Then $\pi_{h, \alpha} \circ \mathrm{W} \circ i_{h, \alpha} \neq(0)$.

(Recall that $\mathfrak{g l}_{n}^{0}$ denotes the trace zero subspace of $\mathfrak{g l}_{n}=$ Lie $\mathrm{GL}_{n}$.) 
Theorem 5.2. - Let $\mathrm{F}$ be an imaginary $C M$ field and let $\mathrm{F}^{+}$denote its maximal totally real subfield. Let $n \in \mathbf{Z}_{\geq 1}$ and let $l>n$ be a prime which is unramified in $\mathrm{F}$. Let

$$
r: \operatorname{Gal}(\overline{\mathrm{F}} / \mathrm{F}) \longrightarrow \mathrm{GL}_{n}\left(\overline{\mathbf{Q}}_{l}\right)
$$

be a continuous irreducible representation with the following properties. Let $\bar{r}$ denote the semisimplification of the reduction of $r$.

1. $r^{c} \cong r^{\vee} \epsilon^{1-n}$.

2. $r$ is unramified at all but finitely many primes.

3. For all places o|l of $\mathrm{F},\left.r\right|_{\mathrm{Gal}\left(\overline{\mathrm{F}}_{v} / \mathrm{F}_{v}\right)}$ is crystalline.

4. There is an element $a \in\left(\mathbf{Z}^{n}\right)^{\operatorname{Hom}\left(\mathrm{F}, \overline{\mathbf{Q}}_{l}\right)}$ such that

- for all $\tau \in \operatorname{Hom}\left(\mathrm{F}, \overline{\mathbf{Q}}_{l}\right)$ we have

$$
l-1-n \geq a_{\tau, 1} \geq \ldots \geq a_{\tau, n} \geq 0
$$

or

$$
l-1-n \geq a_{\tau c, 1} \geq \ldots \geq a_{\tau c, n} \geq 0
$$

- for all $\tau \in \operatorname{Hom}\left(\mathrm{F}, \overline{\mathbf{Q}}_{l}\right)$ and all $i=1, \ldots, n$

$$
a_{\tau c, i}=-a_{\tau, n+1-i}
$$

- for all $\tau \in \operatorname{Hom}\left(\mathrm{F}, \overline{\mathbf{Q}}_{l}\right)$ above a prime $v \mid l$ of $\mathrm{F}$,

$$
\operatorname{dim}_{\overline{\mathbf{Q}} l} \operatorname{gr}^{i}\left(r \otimes_{\tau, \mathrm{F}_{v}} \mathrm{~B}_{\mathrm{DR}}\right)^{\mathrm{Gal}\left(\overline{\mathrm{F}}_{v} / \mathrm{F}_{v}\right)}=0
$$

unless $i=a_{\tau, j}+n-j$ for some $j=1, \ldots, n$ in which case

$$
\operatorname{dim}_{\overline{\mathbf{Q}}_{l}} \operatorname{grr}^{i}\left(r \otimes_{\tau, \mathrm{F}_{v}} \mathrm{~B}_{\mathrm{DR}}\right)^{\mathrm{Gal}\left(\overline{\mathrm{F}}_{v} / \mathrm{F}_{v}\right)}=1 .
$$

5. There is a non-empty finite set $\mathrm{S}=\mathrm{S}_{1} \coprod \mathrm{S}_{2}$ of places of $\mathrm{F}$ not dividing $l$ and for each $v \in \mathrm{S}$ a square integrable representation $\rho_{v}$ of $\mathrm{GL}_{n}\left(\mathrm{~F}_{v}\right)$ over $\overline{\mathbf{Q}}_{l}$ such that

$$
\begin{gathered}
\left.r\right|_{\mathrm{Gal}\left(\overline{\mathrm{F}}_{v} / \mathrm{F}_{v}\right)} ^{\mathrm{ss}}=r_{l}\left(\rho_{v}\right)^{\vee}(1-n)^{\mathrm{ss}} . \\
\text { If } \rho_{v}=\operatorname{Sp}_{m_{v}}\left(\rho_{v}^{\prime}\right) \text { then set } \\
\tilde{r}_{v}=r_{l}\left(\left(\rho_{v}^{\prime}\right)^{\vee}||^{\left(n / m_{v}-1\right)\left(1-m_{v}\right) / 2}\right) .
\end{gathered}
$$

Note that $\left.r\right|_{\mathrm{Gal}\left(\overline{\mathrm{F}}_{v} / \mathrm{F}_{v}\right)}$ has a unique filtration $\mathrm{Fil}_{v}^{j}$ such that

$$
\left.\operatorname{gr}_{v}^{j} r\right|_{\mathrm{Gal}\left(\overline{\mathrm{F}}_{v} / \mathrm{F}_{v}\right)} \cong \widetilde{r}_{v} \epsilon^{j}
$$

for $j=0, \ldots, m_{v}-1$ and equals (0) otherwise. 
For $v \in \mathrm{S}_{1}$ we assume that $m_{v}=n$.

For $v \in \mathrm{S}_{2}$ we assume that $\tilde{r}_{v}$ has irreducible reduction $\bar{r}_{v}$. Then the restriction $\left.\bar{r}\right|_{\mathrm{Gal}\left(\overline{\mathrm{F}}_{v} / \mathrm{F}_{v}\right)}$ inherits a filtration $\overline{\mathrm{Fil}}_{v}^{j}$ with

$$
\overline{\operatorname{gr}}_{v}^{j} \bar{r}_{\mathrm{Gal}\left(\overline{\mathrm{F}}_{v} / \mathrm{F}_{v}\right)} \cong \bar{r}_{v} \epsilon^{j}
$$

for $j=0, \ldots, m_{v}-1$. We also suppose that for $v \in \mathrm{S}_{2}$ and for $j=1, \ldots, m_{v}$ we have

$$
\bar{r}_{v} ¥ \bar{r}_{v} \epsilon^{i}
$$

6. $\overline{\mathrm{F}}^{\mathrm{kerad} \bar{r}}$ does not contain $\mathrm{F}\left(\zeta_{l}\right)$.

7. The image $\bar{r}\left(\mathrm{Gal}\left(\overline{\mathrm{F}} / \mathrm{F}\left(\zeta_{l}\right)\right)\right)$ is big in the sense recalled above.

8. The representation $\bar{r}$ is irreducible and automorphic of weight a and type $\left\{\rho_{v}\right\}_{v \in \mathrm{S}}$ with $\mathrm{S} \neq \emptyset$.

Then $r$ is automorphic of weight $a$ and type $\left\{\rho_{v}\right\}_{v \in \mathrm{S}}$ and level prime to $l$.

Proof. - Choose a CM field L/F with the following properties

- $\mathrm{L}=\mathrm{L}^{+} \mathrm{E}$ with $\mathrm{E}$ an imaginary quadratic field and $\mathrm{L}^{+}$totally real.

$-\mathrm{L} / \mathrm{F}$ is Galois and soluble.

- L is linearly disjoint from $\overline{\mathrm{F}}^{\mathrm{ker} \bar{r}}\left(\zeta_{l}\right)$ over $\mathrm{F}$.

$-l$ splits in $\mathrm{E}$ and is unramified in $\mathrm{L}$.

- All primes in $\mathrm{S}$ split completely in $\mathrm{L} / \mathrm{F}$ and in $\mathrm{L} / \mathrm{L}^{+}$.

By Lemma 4.1 .5 of [CHT] we can find an embedding $\tau_{\mathrm{E}}: \mathrm{E} \hookrightarrow \mathbf{Q}_{l}$, an algebraic character $\psi: \operatorname{Gal}(\overline{\mathrm{L}} / \mathrm{L}) \rightarrow \overline{\mathbf{Q}}_{l}^{\times}$and an element $a^{\prime} \in\left(\mathbf{Z}^{n}\right)^{\operatorname{Hom}\left(\mathrm{L}, \overline{\mathbf{Q}}_{l}\right)}$ such that

$-\psi^{c}=\psi^{-1}$;

- $\psi$ is crystalline at all places above $l$;

- for all $\tau \in \operatorname{Hom}\left(\mathrm{L}, \overline{\mathbf{Q}}_{l}\right)$ extending $\tau_{\mathrm{E}}$ we have

$$
l-1-n \geq a_{\tau, 1}^{\prime} \geq \ldots \geq a_{\tau, n}^{\prime} \geq 0
$$

- for all $\tau \in \operatorname{Hom}\left(\mathrm{F}, \overline{\mathbf{Q}}_{l}\right)$ and all $i=1, \ldots, n$

$$
a_{\tau c, i}^{\prime}=-a_{\tau, n+1-i}^{\prime}
$$

- for all $\tau \in \operatorname{Hom}\left(\mathrm{F}, \overline{\mathbf{Q}}_{l}\right)$ above a prime $v \mid l$ of $\mathrm{F}$,

$$
\operatorname{dim}_{\overline{\mathbf{Q}}_{l}} \operatorname{gr}^{i}\left((r \otimes \psi) \otimes_{\tau, \mathrm{F}_{v}} \mathrm{~B}_{\mathrm{DR}}\right)^{\mathrm{Gal}\left(\overline{\mathrm{F}}_{v} / \mathrm{F}_{v}\right)}=0
$$

unless $i=a_{\tau, j}^{\prime}+n-j$ for some $j=1, \ldots, n$ in which case

$$
\operatorname{dim}_{\overline{\mathbf{Q}}_{l}} \operatorname{gr}^{i}\left((r \otimes \psi) \otimes_{\tau, \mathrm{F}_{v}} \mathrm{~B}_{\mathrm{DR}}\right)^{\operatorname{Gal}\left(\overline{\mathrm{F}}_{v} / \mathrm{F}_{v}\right)}=1 .
$$


Using Lemmas 4.1.3 and 4.2.2 of [CHT] and Theorem 4.2 of [AC] we see that we may reduce to the case where $\mathrm{F}$ contains an imaginary quadratic field $\mathrm{E}$ with an embedding $\tau_{\mathrm{E}}: \mathrm{E} \hookrightarrow \mathbf{Q}_{l}$, such that

$$
l-1-n \geq a_{\tau, 1} \geq \ldots \geq a_{\tau, n} \geq 0
$$

for all $\tau: \mathrm{F} \hookrightarrow \overline{\mathbf{Q}}_{l}$ extending $\tau_{\mathrm{E}}$. (Replace $\mathrm{F}$ by L.) We may also assume that $\mathrm{S}_{1} \cup c \mathrm{~S}_{1}$ and $\mathrm{S}_{2} \cup c \mathrm{~S}_{2}$ are disjoint.

Now suppose that $\bar{r}=\bar{r}_{l, l}(\pi)$, where $\imath: \overline{\mathbf{Q}}_{l} \stackrel{\sim}{\rightarrow} \mathbf{C}$ and where $\pi$ is a RACSDC automorphic representation of $\mathrm{GL}_{n}\left(\mathbf{A}_{\mathrm{F}}\right)$ of weight $l_{*} a$ and type $\left\{l \rho_{v}\right\}_{v \in \mathrm{S}}$ and with $\pi_{l}$ unramified. Let $\mathrm{S}_{l}$ denote the primes of $\mathrm{F}$ above $l$. Let $\mathrm{R}$ denote the primes of $\mathrm{F}$ outside $\mathrm{S}^{c} \cup \mathrm{S} \cup \mathrm{S}_{l}$ at which $r$ or $\pi$ is ramified. Because $\overline{\mathrm{F}}^{\mathrm{ker} a \mathrm{r}}$ does not contain $\mathrm{F}\left(\zeta_{l}\right)$, we can choose a prime $v_{1}$ of $\mathrm{F}$ with the following properties

$-v_{1} \notin \mathrm{R} \cup \mathrm{S}_{l} \cup \mathrm{S} \cup \mathrm{S}^{c}$,

- $v_{1}$ is unramified over a rational prime $p$ for which $\left[\mathrm{F}\left(\zeta_{p}\right): \mathrm{F}\right]>n$,

- $v_{1}$ does not split completely in $\mathrm{F}\left(\zeta_{l}\right)$,

$-\operatorname{ad} \bar{r}\left(\operatorname{Frob}_{v_{1}}\right)=1$.

(We will use primes above $v_{1}$ as auxiliary primes to augment the level so that the open compact subgroups of the finite adelic points of certain unitary groups we consider will be sufficiently small. The properties of $v_{1}$ will ensure that the Galois deformation problems we consider will not change when we allow ramification at primes above $v_{1}$.)

Choose a totally real field $\mathrm{L}^{+} / \mathrm{F}^{+}$with the following properties

$-4 \mid\left[\mathrm{L}^{+}: \mathrm{F}^{+}\right]$.

$-\mathrm{L}^{+} / \mathrm{F}^{+}$is Galois and soluble.

$-\mathrm{L}=\mathrm{L}^{+} \mathrm{E}$ is everywhere unramified over $\mathrm{L}^{+}$.

- $\mathrm{L}$ is linearly disjoint from $\overline{\mathrm{F}}^{\mathrm{ker} \bar{r}}\left(\zeta_{l}\right)$ over $\mathrm{F}$.

$-l$ is unramified in $\mathrm{L}$.

- All primes of $\mathrm{L}$ above $\mathrm{R} \cup \mathrm{S} \cup\left\{v_{1}\right\}$ are split over $\mathrm{L}^{+}$.

- All primes of $\mathrm{S}_{2} \cup c \mathrm{~S}_{2} \cup\left\{v_{1}, c v_{1}\right\}$ split completely in $\mathrm{L} / \mathrm{F}$.

- Let $\pi_{\mathrm{L}}$ denote the base change of $\pi$ to $\mathrm{L}$. If $v$ is a place of $\mathrm{L}$ above $\mathrm{R} \cup \mathrm{S}_{1} \cup{ }_{c} \mathrm{~S}_{1}$ then $\mathbf{N} v \equiv 1 \bmod l$ and $\bar{r} \operatorname{Gal}(\overline{\mathrm{L}} / \mathrm{L})=\left\{1_{n}\right\}$ and $\left.r\right|_{\mathrm{Gal}(\overline{\mathrm{L}} / \mathrm{L})} ^{\mathrm{ss}}=1$ and $\pi_{\mathrm{L}, v}^{\operatorname{Iw}(v)} \neq(0)$.

- If $\mathrm{S}\left(\mathrm{L}^{+}\right)$denotes the set of primes of $\mathrm{L}^{+}$whose restriction to $\mathrm{F}^{+}$lies below an element of $\mathrm{S}$, then $2 \mid \# \mathrm{~S}(\mathrm{~L})$.

Let $a_{\mathrm{L}} \in\left(\mathbf{Z}^{n}\right)^{\operatorname{Hom}\left(\mathrm{L}, \overline{\mathbf{Q}}_{l}\right)}$ be defined by $a_{\mathrm{L}, \tau}=a_{\left.\tau\right|_{\mathrm{F}}}$. Choose a division algebra $\mathrm{B} / \mathrm{L}$ and an involution $\ddagger$ of $\mathrm{B}$ as at the start of Section 4 , with $\mathrm{S}(\mathrm{B})=\mathrm{S}\left(\mathrm{L}^{+}\right)$. Let $\mathrm{S}_{l}\left(\mathrm{~L}^{+}\right)$denote the primes of $\mathrm{L}^{+}$above $l$, let $\mathrm{R}\left(\mathrm{L}^{+}\right)$denote the primes of $\mathrm{L}^{+}$ lying above the restriction to $\mathrm{F}^{+}$of an element of $\mathrm{R}$ and let $\mathrm{S}_{a}\left(\mathrm{~L}^{+}\right)$denote the 
primes of $\mathrm{L}^{+}$above $\left.v_{1}\right|_{\mathrm{F}^{+}}$. Let $\mathrm{T}\left(\mathrm{L}^{+}\right)=\mathrm{S}\left(\mathrm{L}^{+}\right) \cup \mathrm{S}_{l}\left(\mathrm{~L}^{+}\right) \cup \mathrm{R}\left(\mathrm{L}^{+}\right) \cup \mathrm{S}_{a}\left(\mathrm{~L}^{+}\right)$. It follows from Proposition 3.3.2 of [CHT] and Theorem 4.1 of this paper that $\left.r\right|_{\mathrm{Gal}(\overline{\mathrm{F}} / \mathrm{L})}$ is automorphic of weight $a_{\mathrm{L}}$ and type $\left\{\rho_{v \mid \mathrm{F}}\right\}_{v \in \mathrm{S}(\mathrm{L})}$ and level prime to $l$. The theorem now follows from Lemma 4.2.2 of [CHT].

As in Section 4.4 of [CHT] we will say a few words about the conditions in this theorem. The first condition ensures that $r$ is conjugate self-dual. Only for such representations will the numerology behind the Taylor-Wiles argument work. Also it is only for such representations that one can work on a unitary group. Indeed whenever one has a cuspidal automorphic representation of $\mathrm{GL}_{n}\left(\mathbf{A}_{\mathrm{F}}\right)$ for which one knows how to construct a Galois representation, that Galois representation will have this property. The second condition should be necessary, i.e. it should hold for any Galois representation associated to an automorphic form. A weakened form of the third condition which required only that these restrictions are de Rham is also expected to be necessary. The stronger form here (requiring the restrictions to be crystalline), the assumption that $l$ is unramified in $\mathrm{F}$ and the bounds on the Hodge-Tate numbers in condition four are all needed so that we can apply the theory of Fontaine and Laffaille to calculate the local deformation rings at primes above $l$. Condition four also requires the Hodge-Tate numbers to be distinct. Otherwise the numerology behind the Taylor-Wiles method would again fail. The fifth condition is there to ensure that the corresponding automorphic form will be discrete series at some places (i.e. those in S). With the current state of the trace formula this is necessary to move automorphic forms between unitary groups and $\mathrm{GL}_{n}$ and also to construct Galois representations for automorphic forms on $\mathrm{GL}_{n}$. There is some choice for the exact form of condition five. At the primes in $\mathrm{S}_{2}$ we impose conditions to make the local deformation problem as simple as possible. At primes in $\mathrm{S}_{1}$ we allow more complicated local deformation rings, but only in a special case. Some common generalisation of these two cases is probably possible, but we have not spent the time to work this out. The sixth condition is to allow us to choose auxiliary primes which can be used to augment the level and ensure that certain level structures we work with are sufficiently small. The seventh condition is to make the Cebotarev argument used in the Taylor-Wiles argument work. It seems to be often satisfied in practice.

Now we turn to the case of a totally real field. Let $\mathrm{F}^{+}$be a totally real field. By a RAESDC (regular, algebraic, essentially self dual, cuspidal) automorphic representation $\pi$ of $\mathrm{GL}_{n}\left(\mathbf{A}_{\mathrm{F}^{+}}\right)$we mean a cuspidal automorphic representation such that

$-\pi^{\vee} \cong \chi \pi$ for some character $\chi:\left(\mathrm{F}^{+}\right)^{\times} \backslash \mathbf{A}_{\mathrm{F}^{+}}^{\times} \rightarrow \mathbf{G}^{\times}$with $\chi_{v}(-1)$ independent of $v \mid \infty$, and

$-\pi_{\infty}$ has the same infinitesimal character as some irreducible algebraic representation of the restriction of scalars from $\mathrm{F}^{+}$to $\mathbf{Q}$ of $\mathrm{GL}_{n}$. 
(If $n=2$ or if $n$ is odd then one does not require the condition that $\chi_{v}(-1)$ is independent of $v \mid \infty$, in the sense that, if the definition is satisfied with some $\chi$ it will also be satisfied for another $\chi^{\prime}$ with $\chi_{v}^{\prime}(-1)$ independent of $v \mid \infty$. We do not know if it is required for even $n>2$. See the start of Section 4.3 of [CHT].)

Let $a \in\left(\mathbf{Z}^{n}\right)^{\operatorname{Hom}\left(\mathrm{F}^{+}, \mathbf{C}\right)}$ satisfy

$$
a_{\tau, 1} \geq \ldots \geq a_{\tau, n} .
$$

Let $\boldsymbol{\Xi}_{a}$ denote the irreducible algebraic representation of $\mathrm{GL}_{n}^{\mathrm{Hom}\left(\mathrm{F}^{+}, \mathbf{C}\right)}$ which is the tensor product over $\tau$ of the irreducible representations of $\mathrm{GL}_{n}$ with highest weights $a_{\tau}$. We will say that a RAESDG automorphic representation $\pi$ of $\mathrm{GL}_{n}\left(\mathbf{A}_{\mathrm{F}}\right)$ has weight $a$ if $\pi_{\infty}$ has the same infinitesimal character as $\Xi_{a}^{\vee}$.

Let $\mathrm{S}$ be a finite set of finite places of $\mathrm{F}^{+}$. For $v \in \mathrm{S}$ let $\rho_{v}$ be an irreducible square integrable representation of $\mathrm{GL}_{n}\left(\mathrm{~F}_{v}^{+}\right)$. We will say that a RAESDC automorphic representation $\pi$ of $\mathrm{GL}_{n}\left(\mathbf{A}_{\mathrm{F}^{+}}\right)$has type $\left\{\rho_{v}\right\}_{v \in \mathrm{S}}$ if for each $v \in \mathrm{S}, \pi_{v}$ is an unramified twist of $\rho_{v}^{\vee}$.

We recall Proposition 4.3.1 of [CHT].

Proposition 5.3. - Let $\boldsymbol{\imath}: \overline{\mathbf{Q}}_{l} \stackrel{\sim}{\rightarrow} \mathbf{C}$. Let $\mathrm{F}^{+}$be a totally real field, $\mathrm{S}$ a finite non-empty set of finite places of $\mathrm{F}^{+}$and, for $v \in \mathrm{S}, \rho_{v}$ a square integrable representation of $\mathrm{GL}_{n}\left(\mathrm{~F}_{v}^{+}\right)$. Let $a \in\left(\mathbf{Z}^{n}\right)^{\mathrm{Hom}\left(\mathrm{F}^{+}, \mathbf{C}\right)}$ be as above. Suppose that $\pi$ is a RAESDC automorphic representation of $\mathrm{GL}_{n}\left(\mathbf{A}_{\mathrm{F}^{+}}\right)$of weight $a$ and type $\left\{\rho_{v}\right\}_{v \in \mathrm{S}}$. Specifically suppose that $\pi^{\vee} \cong \pi \chi$ where $\chi: \mathbf{A}_{\mathrm{F}^{+}}^{\times} /\left(\mathrm{F}^{+}\right)^{\times} \rightarrow \mathbf{G}^{\times}$. Then there is a continuous semisimple representation

$$
r_{l, l}(\pi): \operatorname{Gal}\left(\overline{\mathrm{F}}^{+} / \mathrm{F}^{+}\right) \longrightarrow \mathrm{GL}_{n}\left(\overline{\mathbf{Q}}_{l}\right)
$$

with the following properties.

1. For every prime vłl of $\mathrm{F}^{+}$we have

$$
\left.r_{l, l}(\pi)\right|_{\mathrm{Gal}\left(\overline{\mathrm{F}}_{v}^{+} / \mathrm{F}_{v}^{+}\right)} ^{\mathrm{ss}}=r_{l}\left(l^{-1} \pi_{v}\right)^{\vee}(1-n)^{\mathrm{ss}} .
$$

2. $r_{l, l}(\pi)^{\vee}=r_{l, l}(\pi) \epsilon^{n-1} r_{l, l}(\chi)$.

3. If $v \mid l$ is a prime of $\mathrm{F}^{+}$then the restriction $\left.r_{l, l}(\pi)\right|_{\mathrm{Gal}_{\left(\mathrm{F}_{v}^{+} / \mathrm{F}_{v}^{+}\right)}}$is potentially semistable, and if $\pi_{v}$ is unramified then it is crystalline.

4. If $v \mid l$ is a prime of $\mathrm{F}^{+}$and if $\tau: \mathrm{F}^{+} \hookrightarrow \overline{\mathbf{Q}}_{l}$ lies above $v$ then

$$
\operatorname{dim}_{\overline{\mathbf{Q}}_{l}} \operatorname{gr}^{i}\left(r_{l, l}(\pi) \otimes_{\tau, \mathrm{F}_{v}^{+}} \mathrm{B}_{\mathrm{DR}}\right)^{\mathrm{Gal}\left(\overline{\mathrm{F}}_{v}^{+} / \mathrm{F}_{v}^{+}\right)}=0
$$

unless $i=a_{\imath \tau, j}+n-j$ for some $j=1, \ldots, n$ in which case

$$
\operatorname{dim}_{\overline{\mathbf{Q}} l} \operatorname{gr}^{i}\left(r_{l, l}(\pi) \otimes_{\tau, \mathrm{F}_{v}^{+}} \mathrm{B}_{\mathrm{DR}}\right)^{\mathrm{Gal}\left(\overline{\mathrm{F}}_{v}^{+} / \mathrm{F}_{v}^{+}\right)}=1 .
$$

The representation $r_{l, l}(\pi)$ can be taken to be valued in $\operatorname{GL}_{n}(\mathscr{O})$ where $\mathscr{O}$ is the ring of integers of some finite extension of $\mathbf{Q}_{l}$. Thus we can reduce it 
modulo the maximal ideal of $\mathscr{O}$ and semisimplify to obtain a continuous semisimple representation

$$
\bar{r}_{l, l}(\pi): \operatorname{Gal}\left(\overline{\mathrm{F}}^{+} / \mathrm{F}^{+}\right) \longrightarrow \mathrm{GL}_{n}\left(\overline{\mathbf{F}}_{l}\right)
$$

which is independent of the choices made.

Let $l: \overline{\mathbf{Q}}_{l} \stackrel{\sim}{\rightarrow} \mathbf{C}$. Suppose that $a \in\left(\mathbf{Z}^{n}\right)^{\operatorname{Hom}\left(\mathrm{F}^{+}, \overline{\mathbf{Q}}_{l}\right)}$ satisfies

$$
a_{\tau, 1} \geq \ldots \geq a_{\tau, n} .
$$

Then we define $\imath_{*} a$ by

$$
\left(l_{*} a\right)_{\imath \tau, i}=a_{\tau, i} .
$$

Suppose that $a \in\left(\mathbf{Z}^{n}\right)^{\operatorname{Hom}\left(\mathrm{F}^{+}, \overline{\mathbf{Q}}_{l}\right)}$ satisfies the conditions of the previous paragraph, that $\mathrm{S}$ is a finite set of finite places of $\mathrm{F}^{+}$not containing any prime above $l$ and that $\rho_{v}$ is a discrete series representation of $\mathrm{GL}_{n}\left(\mathrm{~F}_{v}^{+}\right)$over $\overline{\mathbf{Q}}_{l}$ for all $v \in \mathrm{S}$. We will call a continuous semisimple representation

$$
r: \operatorname{Gal}\left(\overline{\mathrm{F}}^{+} / \mathrm{F}^{+}\right) \longrightarrow \mathrm{GL}_{n}\left(\overline{\mathbf{Q}}_{l}\right)
$$

(resp.

$$
\left.\bar{r}: \operatorname{Gal}\left(\overline{\mathrm{F}}^{+} / \mathrm{F}^{+}\right) \longrightarrow \mathrm{GL}_{n}\left(\overline{\mathbf{F}}_{l}\right)\right)
$$

automorphic of weight $a$ and type $\left\{\rho_{v}\right\}_{v \in \mathrm{S}}$ if there is an isomorphism $\boldsymbol{l}: \overline{\mathbf{Q}}_{l} \stackrel{\sim}{\rightarrow} \mathbf{C}$ and a RAESDG automorphic representation $\pi$ of $\mathrm{GL}_{n}\left(\mathbf{A}_{\mathrm{F}^{+}}\right)$of weight $\boldsymbol{l}_{*} a$ and type $\left\{l \rho_{v}\right\}_{v \in \mathrm{S}}$ (resp. and with $\pi_{l}$ unramified) such that $r \cong r_{l, l}(\pi)$ (resp. $\bar{r} \cong \bar{r}_{l, l}(\pi)$ ). We will say that $r$ is automorphic of weight $a$ and type $\left\{\rho_{v}\right\}_{v \in S}$ and level prime to $l$ if there is an isomorphism $\imath: \overline{\mathbf{Q}}_{l} \stackrel{\sim}{\rightarrow} \mathbf{C}$ and a RAESDG automorphic representation $\pi$ of $\mathrm{GL}_{n}\left(\mathbf{A}_{\mathrm{F}^{+}}\right)$of weight $\boldsymbol{l}_{*} a$ and type $\left\{l \rho_{v}\right\}_{v \in \mathrm{S}}$ and with $\pi_{l}$ unramified such that $r \cong r_{l, l}(\pi)$.

Theorem 5.4. - Let $\mathrm{F}^{+}$be a totally real field. Let $n \in \mathbf{Z}_{\geq 1}$ and let $l>n$ be a prime which is unramified in $\mathrm{F}^{+}$. Let

$$
r: \operatorname{Gal}\left(\overline{\mathrm{F}}^{+} / \mathrm{F}^{+}\right) \longrightarrow \mathrm{GL}_{n}\left(\overline{\mathbf{Q}}_{l}\right)
$$

be a continuous irreducible representation with the following properties. Let $\bar{r}$ denote the semisimplification of the reduction of $r$.

1. $r^{\vee} \cong r \epsilon^{n-1} \chi$ for some character $\chi: \mathrm{Gal}\left(\overline{\mathrm{F}}^{+} / \mathrm{F}^{+}\right) \longrightarrow \overline{\mathbf{Q}}_{l}^{\times}$with $\chi\left(c_{v}\right)$ independent of $v \mid \infty$. (Here $c_{v}$ denotes a complex conjugation at v.)

2. $r$ ramifies at only finitely many primes. 
3. For all places $v \mid l$ of $\mathrm{F}^{+},\left.r\right|_{\mathrm{Gal}\left(\overline{\mathrm{F}}_{v}^{+} / \mathrm{F}_{v}^{+}\right)}$is crystalline.

4. There is an element $a \in\left(\mathbf{Z}^{n}\right)^{\operatorname{Hom}\left(\mathrm{F}^{+}, \overline{\mathbf{Q}}_{l}\right)}$ such that - for all $\tau \in \operatorname{Hom}\left(\mathrm{F}^{+}, \overline{\mathbf{Q}}_{l}\right)$ we have

$$
l-1-n+a_{\tau, n} \geq a_{\tau, 1} \geq \ldots \geq a_{\tau, n}
$$

- for all $\tau \in \operatorname{Hom}\left(\mathrm{F}^{+}, \overline{\mathbf{Q}}_{l}\right)$ above a prime v|l of $\mathrm{F}^{+}$,

$$
\operatorname{dim}_{\overline{\mathbf{Q}}_{l}} \operatorname{gr}^{i}\left(r \otimes_{\tau, \mathrm{F}_{v}^{+}} \mathrm{B}_{\mathrm{DR}}\right)^{\mathrm{Gal}\left(\overline{\mathrm{F}}_{v}^{+} / \mathrm{F}_{v}^{+}\right)}=0
$$

unless $i=a_{\tau, j}+n-j$ for some $j=1, \ldots, n$ in which case

$$
\operatorname{dim}_{\overline{\mathbf{Q}}_{l}} \operatorname{gr}^{i}\left(r \otimes_{\tau, \mathrm{F}_{v}^{+}} \mathrm{B}_{\mathrm{DR}}\right)^{\mathrm{Gal}\left(\overline{\mathrm{F}}_{v}^{+} / \mathrm{F}_{v}^{+}\right)}=1 .
$$

5. There is a non-empty finite set $\mathrm{S}=\mathrm{S}_{1} \bigsqcup \mathrm{S}_{2}$ of places of $\mathrm{F}^{+}$not dividing $l$ and for each $v \in \mathrm{S}$ a square integrable representation $\rho_{v}$ of $\mathrm{GL}_{n}\left(\mathrm{~F}_{v}^{+}\right)$over $\overline{\mathbf{Q}}_{l}$ such that

$$
\begin{gathered}
\left.r\right|_{\mathrm{Gal}\left(\overline{\mathrm{F}}_{v}^{+} / \mathrm{F}_{v}^{+}\right)} ^{\mathrm{ss}}=r_{l}\left(\rho_{v}\right)^{\vee}(1-n)^{\mathrm{Ss}} . \\
\text { If } \rho_{v}=\operatorname{Sp}_{m_{v}}\left(\rho_{v}^{\prime}\right) \text { then set } \\
\tilde{r}_{v}=r_{l}\left(\left(\rho_{v}^{\prime}\right)^{\vee}||^{\left(n / m_{v}-1\right)\left(1-m_{v}\right) / 2}\right) .
\end{gathered}
$$

Note that $\left.r\right|_{\mathrm{Gal}\left(\overline{\mathrm{F}}_{v}^{+} / \mathrm{F}_{v}^{+}\right)}$has a unique filtration $\mathrm{Fil}_{v}^{j}$ such that

$$
\left.\operatorname{gr}_{v}^{j} r\right|_{\mathrm{Gal}\left(\overline{\mathrm{F}}_{v} / \mathrm{F}_{v}\right)} \cong \widetilde{r}_{v} \epsilon^{j}
$$

for $j=0, \ldots, m_{v}-1$ and equals (0) otherwise.

For $v \in \mathrm{S}_{1}$ we assume that $m_{v}=n$.

For $v \in \mathrm{S}_{2}$ we assume that $\tilde{r}_{v}$ has irreducible reduction $\bar{r}_{v}$. Then the restriction $\left.\bar{r}\right|_{\left.\mathrm{Gal}_{(} \overline{\mathrm{F}}_{v}^{+} / \mathrm{F}_{v}^{+}\right)}$inherits a filtration $\overline{\mathrm{Fil}}_{v}^{j}$ with

$$
\left.\overline{\operatorname{gr}}_{v}^{j} \bar{r}\right|_{\mathrm{Gal}\left(\overline{\mathrm{F}}_{v}^{+} / \mathrm{F}_{v}^{+}\right)} \cong \bar{r}_{v} \epsilon^{j}
$$

for $j=0, \ldots, m_{v}-1$. We also suppose that for $v \in \mathrm{S}_{2}$ and for $j=1, \ldots, m_{v}$ we have

$$
\bar{r}_{v} \not \bar{r}_{v} \epsilon^{i}
$$

6. $\left(\overline{\mathrm{F}}^{+}\right)^{\mathrm{ker} \mathrm{ad} \bar{r}}$ does not contain $\mathrm{F}^{+}\left(\zeta_{l}\right)$.

7. The image $\bar{r}\left(\mathrm{Gal}\left(\overline{\mathrm{F}}^{+} / \mathrm{F}^{+}\left(\zeta_{l}\right)\right)\right)$ is big (as defined just before Theorem 5.2).

8. $\bar{r}$ is irreducible and automorphic of weight $a$ and type $\left\{\rho_{v}\right\}_{v \in \mathrm{S}}$ with $\mathrm{S} \neq \varnothing$.

Then $r$ is automorphic of weight $a$ and type $\left\{\rho_{v}\right\}_{v \in \mathrm{S}}$ and level prime to $l$. 
Proof. - Choose an imaginary CM field F with maximal totally real subfield $\mathrm{F}^{+}$such that

- all primes above $l$ split in $\mathrm{F} / \mathrm{F}^{+}$,

- all primes in $\mathrm{S}$ split in $\mathrm{F} / \mathrm{F}^{+}$, and

$-\mathrm{F}$ is linearly disjoint from $\left(\overline{\mathrm{F}}^{+}\right)^{\operatorname{ker} \bar{r}}\left(\zeta_{l}\right)$ over $\mathrm{F}^{+}$.

Choose an algebraic character

$$
\psi: \operatorname{Gal}\left(\overline{\mathrm{F}}^{+} / \mathrm{F}\right) \longrightarrow \overline{\mathbf{Q}}_{l}^{\times}
$$

such that

$-\left.\chi\right|_{\mathrm{Gal}\left(\overline{\mathrm{F}}^{+} / \mathrm{F}\right)}=\psi \psi^{c}$,

- $\psi$ is crystalline above $l$, and

- for each $\tau: \mathrm{F}^{+} \hookrightarrow \overline{\mathbf{Q}}_{l}$ there exists an extension $\tilde{\tau}: \mathrm{F} \hookrightarrow \overline{\mathbf{Q}}_{l}$ such that

$$
\operatorname{gr}^{-a_{\tau, n}}\left(\overline{\mathbf{Q}}_{l}(\psi) \otimes_{\tilde{\tau}, \mathrm{F}_{v(\tau)}} \mathrm{B}_{\mathrm{DR}}\right)^{\mathrm{Gal}\left(\overline{\mathrm{F}}_{v(\tau)} / \mathrm{F}_{v(\tau)}\right)} \neq(0),
$$

where $v(\tilde{\tau})$ is the place of $\mathrm{F}$ above $l$ determined by $\tilde{\tau}$.

(This is possible by Lemma 4.1 .5 of [CHT].) Now apply Theorem 5.2 to to the twist $\left.r\right|_{\mathrm{Gal}\left(\overline{\mathrm{F}}^{+} / \mathrm{F}\right)} \psi$ and the current theorem follows by Lemma 4.3 .3 of [CHT].

As the conditions of this theorem are a bit complicated we give a special case as a corollary.

Corollary 5.5. - Let $n \in \mathbf{Z}_{\geq 1}$ be even and let $l>\max \{3, n\}$ be a prime. Let $\mathrm{S}$ be a finite non-empty set of primes such that if $q \in \mathrm{S}$ then $q \neq l$ and $q^{i} \neq \equiv 1 \bmod l$ for $i=1, \ldots, n$. Let

$$
r: \operatorname{Gal}(\overline{\mathbf{Q}} / \mathbf{Q}) \longrightarrow \operatorname{GSp}_{n}\left(\mathbf{Z}_{l}\right)
$$

be a continuous irreducible representation with the following properties.

1. $r$ ramifies at only finitely many primes.

2. $\left.r\right|_{\mathrm{Gal}\left(\overline{\mathbf{Q}}_{l} / \mathbf{Q}_{l}\right)}$ is crystalline, and $\operatorname{dim}_{\mathbf{Q}_{l}} \operatorname{gr}^{i}\left(r \otimes_{\mathbf{Q}_{l}} \mathrm{~B}_{\mathrm{DR}}\right)^{\mathrm{Gal}\left(\overline{\mathbf{Q}}_{l} / \mathbf{Q}_{l}\right)}=0$ unless $i \in$ $\{0,1, \ldots, n-1\}$, in which case it has dimension 1 .

3. If $q \in \mathrm{S}$ then $\left.r\right|_{\mathrm{G}_{\mathbf{Q}_{q}}} ^{\mathrm{ss}}$ is unramified and $\left.r\right|_{\mathrm{G}_{\mathbf{Q}_{q}}} ^{\mathrm{ss}}\left(\right.$ Frob $\left._{q}\right)$ has eigenvalues $\left\{\alpha q^{i}: i=\right.$ $0,1, \ldots, n-1\}$ for some $\alpha$.

4. The image of $r \bmod l$ contains $\operatorname{Sp}_{n}\left(\mathbf{F}_{l}\right)$.

5. $r \bmod l$ is automorphic of weight 0 and type $\left\{\mathrm{Sp}_{n}(1)\right\}_{q \in \mathrm{S}}$.

Then $r$ is automorphic of weight 0 and type $\left\{\operatorname{Sp}_{n}(1)\right\}_{\{q\}}$ and level prime to $l$. 
Proof. - Let $\bar{r}=r \bmod l$. As $\operatorname{PSp}_{n}\left(\mathbf{F}_{l}\right)$ is simple, the maximal abelian quotient of $\operatorname{ad} \bar{r}\left(\mathrm{G}_{\mathbf{Q}}\right)$ is

$$
\bar{r}\left(\mathrm{G}_{\mathbf{Q}}\right) /\left(\bar{r}\left(\mathrm{G}_{\mathbf{Q}}\right) \cap \mathbf{F}_{l}^{\times}\right) \operatorname{Sp}_{n}\left(\mathbf{F}_{l}\right) \subset \operatorname{PGSp}_{n}\left(\mathbf{F}_{l}\right) / \operatorname{PSp}_{n}\left(\mathbf{F}_{l}\right) \stackrel{\sim}{\longrightarrow}\left(\mathbf{F}_{l}^{\times}\right) /\left(\mathbf{F}_{l}^{\times}\right)^{2} .
$$

Thus $\overline{\mathbf{Q}}^{\mathrm{kerad} \bar{r}}$ does not contain $\mathbf{Q}\left(\zeta_{l}\right)$.

The corollary now follows from Lemma 2.5.5 of [CHT] and Theorem 5.4 of this paper.

As in Section 5.6 of [CHT], we would like to apply Theorems 5.2 and 5.4 in situations where one knows that $\bar{r}$ is automorphic. One such case is where $\bar{r}: \mathrm{Gal}(\overline{\mathrm{F}} / \mathrm{F}) \rightarrow \mathrm{GL}_{n}(k)$ is induced from a (suitable) character over some cyclic extension. However it will be useful to have such a theorem when $\rho_{v}$ is Steinberg for $v \in \mathrm{S}$. Because the lift of $\bar{r}$ which we know to be automorphic is an automorphic induction it can not be Steinberg at any finite place (although it can be cuspidal at a finite place). Thus we have a problem in applying Theorems 5.2 or 5.4 directly. We shall get round this by applying Proposition 2.7.4 of [CHT] to construct a second lift $r_{1}$ of $\bar{r}$ which is Steinberg at $v \in \mathrm{S}$, but which is also cuspidal at some other finite places $\mathrm{S}^{\prime}$. We first show that $r_{1}$ is automorphic using the places in $\mathrm{S}^{\prime}$. The result is that we succeed in 'raising the level' for the automorphicity of $\bar{r}$. We can then apply Theorem 5.2 or 5.4 a second time. A further complication arises because we want to treat $\bar{r}$ which do not look as if they could have a lift which is cuspidal at any finite place. We will do so under an assumption that $\bar{r}$ extends to a representation of $\operatorname{Gal}(\overline{\mathbf{Q}} / \mathbf{Q})$ which looks as if it could have a lift which is cuspidal at some finite place.

More precisely we will consider the following situation.

- M/Q is a Galois imaginary $\mathrm{CM}$ field of degree $n$ with $\operatorname{Gal}(\mathbf{M} / \mathbf{Q})$ cyclic generated by an element $\tau$.

$-l>1+(n-1)\left((n+2)^{n / 2}-(n-2)^{n / 2}\right) / 2^{n-1}\left(\right.$ e.g. $\left.l>8((n+2) / 4)^{1+n / 2}\right)$ is a prime which splits completely in $\mathrm{M}$ and is $\equiv 1 \bmod n$.

$-p$ is a rational prime which is inert and unramified in $\mathrm{M}$.

$-q \neq l$ is a rational prime, which splits completely in $\mathrm{M}$ and which satisfies $q^{i} \not \equiv 1 \bmod l$ for $i=1, \ldots, n-1$.

$-\bar{\theta}: \operatorname{Gal}(\overline{\mathbf{Q}} / \mathrm{M}) \longrightarrow \overline{\mathbf{F}}_{l}^{\times}$is a continuous character such that

$-\bar{\theta} \bar{\theta}^{c}=\epsilon^{1-n}$

- there exists a prime $w \mid l$ of $\mathrm{M}$ such that for $i=0, \ldots, n / 2-1$ we have $\left.\bar{\theta}\right|_{\mathrm{I}_{\tau^{i} w}}=\epsilon^{-i}$;

- if $v_{1}, \ldots, v_{n}$ are the primes of $\mathrm{M}$ above $q$ then $\left\{\bar{\theta}\left(\right.\right.$ Frob $\left.\left._{v_{i}}\right)\right\}$ equals $\left\{\alpha_{q} q^{-j}\right.$ : $j=0, \ldots, n-1\}$ for some $\alpha_{q} \in \overline{\mathbf{F}}_{l}^{\times}$;

$-\left.\bar{\theta}\right|_{\mathrm{Gal}\left(\overline{\mathrm{M}}_{p} / \mathrm{M}_{p}\right)} \neq\left.\bar{\theta}^{\tau^{j}}\right|_{\mathrm{Gal}\left(\overline{\mathrm{M}}_{p} / \mathrm{M}_{p}\right)}$ for $j=1, \ldots, n-1$.

Let $\mathrm{S}(\bar{\theta})$ denote the set of rational primes above which $\mathrm{M}$ or $\bar{\theta}$ is ramified. 
- E/Q is an imaginary quadratic field linearly disjoint from the Galois closure of $\overline{\mathrm{M}}^{\mathrm{ker} \bar{\theta}}\left(\zeta_{l}\right) / \mathbf{Q}$ in which every element of $\mathrm{S}(\bar{\theta}) \cup\{l, q, p\}$ splits; and such that the class number of $\mathrm{E}$ is not divisible by $l$.

A referee asked the good question: are there any examples where all these conditions are met? The answer is 'yes'. One example is given in the proof of Theorem 3.1 of [HSBT].

Theorem 5.6. - Keep the notation and assumptions listed above. Let $\mathrm{F} / \mathrm{F}_{0}$ be a Galois extension of imaginary CM fields with $\mathrm{F}$ linearly disjoint from the normal closure of $\overline{\mathrm{M}}^{\mathrm{ker} \bar{\theta}}\left(\zeta_{l}\right)$ over $\mathbf{Q}$. Assume that $l$ is unramified in $\mathrm{F}$ and that there is a prime $v_{p, 0}$ of $\mathbf{F}_{0}$ split above $p$. Let

$$
r: \mathrm{Gal}(\overline{\mathrm{F}} / \mathrm{F}) \longrightarrow \mathrm{GL}_{n}\left(\overline{\mathbf{Q}}_{l}\right)
$$

be a continuous irreducible representation with the following properties. Let $\bar{r}$ denote the semisimplification of the reduction of $r$.

$$
\text { 1. } \bar{r} \cong \operatorname{Ind}_{\mathrm{Gal}(\overline{\mathrm{F}} / \mathrm{FM})}^{\mathrm{Ga} / \bar{\theta} / \mathrm{Gal}(\overline{\mathrm{F}} / \mathrm{FM})} .
$$

2. $r^{c} \cong r^{\vee} \epsilon^{1-n}$.

3. $r$ ramifies at only finitely many primes.

4. For all places $v \mid l$ of $\mathrm{F},\left.r\right|_{\mathrm{Gal}\left(\overline{\mathrm{F}}_{v} / \mathrm{F}_{v}\right)}$ is crystalline.

5. For all $\tau \in \operatorname{Hom}\left(\mathrm{F}, \overline{\mathbf{Q}}_{l}\right)$ above a prime $v \mid l$ of $\mathrm{F}$,

$$
\operatorname{dim}_{\overline{\mathbf{Q}},} \operatorname{gr}^{i}\left(r \otimes_{\tau, \mathrm{F}_{v}} \mathrm{~B}_{\mathrm{DR}}\right)^{\mathrm{Gal}\left(\overline{\mathrm{F}}_{v} / \mathrm{F}_{v}\right)}=1
$$

for $i=0, \ldots, n-1$ and $=0$ otherwise.

6. There is a place $v_{q}$ of $\mathrm{F}$ above $q$ such that $\left.r\right|_{\mathrm{Gal}\left(\mathrm{F}_{v_{q}} / \mathrm{F}_{v_{q}}\right)}$ is unramified and $\left.r\right|_{\mathrm{Ga}\left(\overline{\mathrm{F}}_{v_{q}} / \mathrm{F}_{v_{q}}\right)} ^{\mathrm{ss}}\left(\operatorname{Frob}_{v_{q}}\right)$ has eigenvalues $\left\{\alpha\left(\# k\left(v_{q}\right)\right)^{j}: j=0, \ldots, n-1\right\}$ for some $\alpha \in \overline{\mathbf{Q}}_{l}^{\times}$.

Then $r$ is automorphic over $\mathrm{F}$ of weight 0 and type $\left\{\mathrm{Sp}_{n}(1)\right\}_{\left\{v_{q}\right\}}$ and level prime to $l$.

Proof. - We repeat, almost verbatim, the proof of Theorem 5.6.1 of [CHT]. Replacing $\mathrm{F}$ by EF if necessary we may suppose that $\mathrm{F} \supset \mathrm{E}$ (see Lemma 4.2.2 of $[\mathrm{CHT}])$.

Choose a continuous character

$$
\theta: \operatorname{Gal}(\overline{\mathrm{M}} / \mathrm{M}) \longrightarrow \mathscr{O}_{\overline{\mathbf{Q}}}^{\times}
$$

such that

$-\theta$ lifts $\bar{\theta}$

$-\theta^{-1}=\epsilon^{n-1} \theta^{c}$

- for $i=0, \ldots, n / 2-1$ we have $\left.\theta\right|_{\mathrm{I}_{\mathrm{M} i_{w}}}=\epsilon^{-i}$; and

- $l \nmid \# \theta\left(\mathrm{I}_{v}\right)$ for all places $v \mid p$ of $\mathrm{M}$. 
(See Lemma 4.1 .6 of [CHT].) We can extend $\left.\theta\right|_{\mathrm{Gal}(\overline{\mathrm{E}} / \mathrm{EM})}$ to a continuous homomorphism

$$
\theta: \operatorname{Gal}\left(\overline{\mathrm{E}} /(\mathrm{EM})^{+}\right) \longrightarrow \mathscr{G}_{1}\left(\mathscr{O}_{\overline{\mathbf{Q}}_{l}}\right)
$$

with $\nu \circ \theta=\epsilon^{1-n}$. We will let $\bar{\theta}$ also denote the reduction

$$
\bar{\theta}: \operatorname{Gal}\left(\overline{\mathrm{E}} /(\mathrm{EM})^{+}\right) \longrightarrow \mathscr{G}_{1}\left(\overline{\mathbf{F}}_{l}\right)
$$

of $\theta$. Consider the pairs $\operatorname{Gal}\left(\overline{\mathrm{E}} /(\mathrm{EM})^{+}\right) \supset \operatorname{Gal}(\overline{\mathrm{E}} /(\mathrm{EM}))$ and $\operatorname{Gal}(\overline{\mathrm{E}} / \mathbf{Q}) \supset \operatorname{Gal}(\overline{\mathrm{E}} / \mathrm{E})$. Set

$$
r_{0}=\operatorname{Ind}_{\mathrm{Gal}\left(\overline{\mathrm{E}} /(\mathrm{EM})^{+}\right)}^{\mathrm{Ga}(\overline{\mathbf{E}}), \epsilon^{1-n}} \theta: \operatorname{Gal}(\overline{\mathrm{E}} / \mathbf{Q}) \longrightarrow \mathscr{G}_{n}\left(\mathscr{O}_{\overline{\mathbf{Q}}_{l}}\right)
$$

Note also that

$$
\left.r_{0}\right|_{\mathrm{Gal}(\overline{\mathrm{E}} / \mathrm{E})}=\left(\left.\left(\operatorname{Ind}_{\mathrm{Gal}(\overline{\mathrm{E}} / \mathrm{M})}^{\mathrm{Gal}(\overline{\mathrm{E}} \mathbf{\mathbf { Q }})} \theta\right)\right|_{\mathrm{Gal}(\overline{\mathrm{E}} / \mathrm{E})}, \epsilon^{1-n}\right) .
$$

By Proposition 2.7.4 of [CHT] there is a continuous homomorphism

$$
r_{1}: \operatorname{Gal}(\overline{\mathrm{E}} / \mathbf{Q}) \longrightarrow \mathscr{G}_{n}\left(\mathscr{O}_{\overline{\mathbf{Q}}_{l}}\right)
$$

with the following properties.

$-r_{1} \quad \operatorname{lifts} \operatorname{Ind}_{\mathrm{Gal}\left(\overline{\mathrm{E}} /(\mathrm{EM})^{+}\right)}^{\mathrm{Gal}(\bar{\theta}),}$

$-v \circ r_{1}=\epsilon^{1-n}$.

- For all places $w \mid l$ of $\mathrm{E},\left.r_{1}\right|_{\mathrm{Gal}\left(\overline{\mathrm{E}}_{w} / \mathrm{E}_{w}\right)}$ is crystalline.

- For all $\tau \in \operatorname{Hom}\left(\mathrm{E}, \overline{\mathbf{Q}}_{l}\right)$ corresponding to prime $w \mid l$,

$$
\operatorname{dim}_{\overline{\mathbf{Q}} l} \operatorname{gr}^{i}\left(r_{1} \otimes_{\tau, \mathrm{E}_{w}} \mathrm{~B}_{\mathrm{DR}}\right)^{\mathrm{Gal}\left(\overline{\mathrm{E}}_{w} / \mathrm{E}_{w}\right)}=1
$$

for $i=0, \ldots, n-1$ and $=0$ otherwise.

$-\left.r_{1}\right|_{\mathrm{Gal}\left(\overline{\mathrm{E}}_{v q} / \mathrm{E}_{v q}\right)} ^{\mathrm{ss}}$ is unramified and $\left.r\right|_{\mathrm{Gal}^{\mathrm{s}}\left(\overline{\mathrm{E}}_{v_{q}} / \mathrm{E}_{v_{q}}\right)} ^{\mathrm{ss}}\left(\mathrm{Frob}_{v_{q} \mid \mathrm{E}}\right)$ has eigenvalues $\left\{\alpha q^{-j}\right.$ :

$j=0, \ldots, n-1\}$ for some $\alpha \in \overline{\mathbf{Q}}_{l}^{\times}$.

$-\left.r_{1}\right|_{\mathrm{Gal}\left(\overline{\mathrm{E}}_{v_{p}} / \mathrm{E}_{v_{p}}\right)}$ is an unramified twist of $\left.\operatorname{Ind}_{\mathrm{Gal}\left(\overline{\mathbf{Q}}_{p} / \mathrm{M}_{p}\right)}^{\mathrm{Gal}\left(\overline{\mathbf{Q}}_{p} / \mathbf{Q}_{p}\right)} \theta\right|_{\mathrm{Gal}\left(\overline{\mathbf{Q}}_{p} / \mathrm{M}_{p}\right)}$.

Let $v_{p}$ be a prime of $\mathrm{F}$ above $v_{p, 0}$ and let $\mathrm{F}_{1} \subset \mathrm{F}$ denote the fixed field of the decomposition group of $v_{p}$ in $\operatorname{Gal}\left(\mathrm{F} / \mathrm{F}_{0}\right)$. Thus $\left.v_{p}\right|_{\mathrm{F}_{1}}$ is split over $p$ and $\mathrm{F} / \mathrm{F}_{1}$ is soluble.

The restriction $\left.r_{0}\right|_{\mathrm{Gal}\left(\overline{\mathrm{E}} / \mathrm{F}_{1}\right)}$ is automorphic of weight 0 , level prime to $l$ and type $\left\{\rho_{p}\right\}_{\left\{v_{p} \mid \mathrm{F}_{1}\right\}}$, for a suitable cuspidal representation $\rho_{p}$ (by Theorem 4.2 of [AC]). Applying Lemma 2.7.5 of [CHT] and Theorem 5.2 above we deduce that $\left.r_{1}\right|_{\mathrm{Gal}\left(\overline{\mathrm{F}} / \mathrm{F}_{1}\right)}$ is automorphic of weight 0 and type $\left\{\rho_{p}\right\}_{\left\{v_{p} \mid \mathrm{F}_{1}\right\}}$ and level prime to $l$. 
It follows from Corollary VII.1.11 of $[\mathrm{HT}]$ that $\left.r_{1}\right|_{\mathrm{Gal}\left(\overline{\mathrm{F}} / \mathrm{F}_{1}\right)}$ is also automorphic of weight 0 and type $\left\{\operatorname{Sp}_{n}(1)\right\}_{\left\{v_{q} \mid \mathrm{F}_{\mathrm{H}}\right\}}$ and level prime to $l$. (The only tempered representations $\pi$ of $\mathrm{GL}_{n}\left(\mathrm{~F}_{1, v_{q} \mid \mathrm{F}_{1}}\right)$ for which $r_{l}(\pi)^{\vee}(1-n)^{\mathrm{ss}}$ unramified and $r_{l}(\pi)^{\vee}(1-n)^{\mathrm{Ss}}\left(\right.$ Frob $\left._{\left.v_{q}\right|_{\mathrm{F}_{1}}}\right)$ has eigenvalues of the form $\left\{\alpha q^{-j}: j=0, \ldots, n-1\right\}$ are unramified twists of $\mathrm{Sp}_{n}(1)$.) From Theorem 4.2 of [AC] we deduce that $\left.r_{1}\right|_{\text {Gal }(\overline{\mathrm{F}} / \mathrm{F})}$ is automorphic of weight 0 and type $\left\{\operatorname{Sp}_{n}(1)\right\}_{\left\{v_{q}\right\}}$ and level prime to $l$. (The base change must be cuspidal as it is square integrable at one place.)

Finally we again apply Theorem 5.2 to deduce that $r$ is automorphic of weight 0 and type $\left\{\rho_{p}\right\}_{\left\{v_{p}\right\}}$ and level prime to $l$. The verification that $\bar{r}\left(\mathrm{G}_{\mathrm{F}^{+}\left(\zeta_{l}\right)}\right)$ is big is exactly as above.

We also have a version for totally real fields.

Theorem 5.7. - Keep the notation and assumptions listed before Theorem 5.6. Let $\mathrm{F}^{+} / \mathrm{F}_{0}^{+}$be a Galois extension of totally real fields with $\mathrm{F}^{+}$linearly disjoint from the Galois closure of $\mathrm{E}\left(\zeta_{l}\right) \overline{\mathrm{M}}^{\mathrm{ker} \bar{\theta}}$ over $\mathbf{Q}$. Suppose that that $l$ is unramified in $\mathrm{F}^{+}$and that there is a prime $v_{p, 0}$ of $\mathrm{F}_{0}^{+}$split over $p$. Let

$$
r: \operatorname{Gal}\left(\overline{\mathrm{F}^{+}} / \mathrm{F}^{+}\right) \longrightarrow \mathrm{GL}_{n}\left(\overline{\mathbf{Q}}_{l}\right)
$$

be a continuous representation such that

$-\left.\bar{r} \cong\left(\operatorname{Ind}_{\mathrm{Gal}(\overline{\mathbf{Q}} / \mathrm{M})}^{\mathrm{Ga}(\overline{\bar{Q}})} \bar{\theta}\right)\right|_{\mathrm{Gal}\left(\overline{\mathbf{Q}} / \mathrm{F}^{+}\right)} ;$

$-r^{\vee} \cong r \epsilon^{n-1}$;

$-r$ is unramified at all but finitely many primes;

- For all places oll of $\mathrm{F}^{+},\left.r\right|_{\mathrm{Gal}\left(\overline{\mathrm{F}}_{v}^{+} / \mathrm{F}_{v}^{+}\right)}$is crystalline.

- For all $\tau \in \operatorname{Hom}\left(\mathrm{F}^{+}, \overline{\mathbf{Q}}_{l}\right)$ above a prime $v \mid l$ of $\mathrm{F}^{+}$,

$$
\operatorname{dim}_{\overline{\mathbf{Q}}_{l}} \operatorname{grr}^{i}\left(r \otimes_{\tau, \mathrm{F}_{v}^{+}} \mathrm{B}_{\mathrm{DR}}\right)^{\mathrm{Gal}\left(\overline{\mathrm{F}}_{v}^{+} / \mathrm{F}_{v}^{+}\right)}=1
$$

for $i=0, \ldots, n-1$ and $=0$ otherwise.

- There is a place $v_{q} \mid q$ of $\mathrm{F}^{+}$such that $\left.r\right|_{\mathrm{Gal}\left(\overline{\mathrm{F}}_{v_{q}}^{+} / \mathrm{F}_{v_{q}}^{+}\right)} ^{\mathrm{ss}}$ is unramified and $\left.r\right|_{\mathrm{Gal}\left(\overline{\mathrm{F}}_{v_{q}}^{+} / \mathrm{F}_{v_{q}}^{+}\right)} ^{\mathrm{ss}}\left(\mathrm{Frob}_{v_{q}}\right)$ has eigenvalues $\left\{\alpha\left(\# k\left(v_{q}\right)\right)^{j}: j=0, \ldots, n-1\right\}$ for some $\alpha \in \overline{\mathbf{Q}}_{l}^{\times}$.

Then $r$ is automorphic over $\mathrm{F}^{+}$of weight 0 and type $\left\{\operatorname{Sp}_{n}(1)\right\}_{\left\{v_{q}\right\}}$ and level prime to $l$.

Proof. - Apply Theorem 5.6 to $\mathrm{F}=\mathrm{F}^{+} \mathrm{E}$ and use Lemma 4.3.3 of [CHT].

\section{Applications}

The proofs of the following results are given in [HSBT]. When [HSBT] was first submitted that paper relied on the conditional theorems proved in Chapter 5 of $[\mathrm{CHT}]$, and so the main theorems of [HSBT] were themselves conditional. 
The proofs of these theorems became unconditional when the present paper was written. For the convenience of the reader we have rewritten [HSBT] so that its results are now stated unconditionally and reference is made to this paper. However, as the proofs of those results were finally completed in this paper, we restate them here.

Let $n \in \mathbf{Z}_{>1}$ be an even integer. If $\mathrm{K}$ is a field of characteristic not dividing $n+1$ and if $t \in \mathrm{K}$ is not an $(n+1)^{\text {th }}$ root of unity then $\mathrm{Y}_{t} / \mathrm{K}$ will denote the $(n-1)$-dimensional smooth projective variety:

$$
\left(\mathrm{X}_{0}^{n+1}+\mathrm{X}_{1}^{n+1}+\ldots+\mathrm{X}_{n}^{n+1}\right)=(n+1) t \mathrm{X}_{0} \mathrm{X}_{1} \ldots \mathrm{X}_{n}
$$

It carries an action of

$$
\mathrm{H}^{\prime}=\operatorname{ker}\left(\mu_{n+1}^{n+1} \stackrel{\prod}{\longrightarrow} \mu_{n+1}\right)
$$

by

$$
\left(\zeta_{0}, \ldots, \zeta_{n}\right):\left(\mathrm{X}_{0}: \ldots: \mathrm{X}_{n}\right) \longmapsto\left(\zeta_{0} \mathrm{X}_{0}: \ldots: \zeta_{n} \mathrm{X}_{n}\right)
$$

(Note that the diagonal copy of $\mu_{n+1}$ acts trivially.) If $l \nmid(n+1)$ is a prime set

$$
\mathrm{V}_{n, l}=\mathrm{H}^{n-1}\left(\mathrm{Y}_{t} \times \overline{\mathrm{K}}, \mathbf{Q}_{l}\right)^{\mathrm{H}^{\prime}}
$$

and

$$
\mathrm{V}_{n}[l]=\mathrm{H}^{n-1}\left(\mathrm{Y}_{t} \times \overline{\mathrm{K}}, \mathbf{F}_{l}\right)^{\mathrm{H}^{\prime}} .
$$

These are $\mathrm{G}_{\mathrm{K}}$-modules of dimension n. (See for instance [HSBT].)

Theorem 6.1. - Suppose that $\mathrm{F}$ is a totally real field and that $n$ is an even positive integer. Suppose also that $l$ is a sufficiently large (depending only on n) rational prime which is unramified in $\mathrm{F}$. Let $v_{q}$ be a prime of $\mathrm{F}$ above a rational prime $q \nmid l(n+1)$.

Suppose also that

$$
r: \operatorname{Gal}(\overline{\mathrm{F}} / \mathrm{F}) \longrightarrow \operatorname{GSp}_{n}\left(\mathbf{Z}_{l}\right)
$$

is a continuous representation with the following properties.

1.r has multiplier $\epsilon_{l}^{1-n}$.

2. $r$ ramifies at only finitely many primes.

3. Let $\bar{r}$ denote the semisimplification of the reduction of $r$. Then the image $\bar{r} \mathrm{Gal}\left(\overline{\mathrm{F}} / \mathrm{F}\left(\zeta_{l}\right)\right)$ is big.

4. $\overline{\mathrm{F}}^{\mathrm{kerad} \bar{r}}$ does not contain $\mathrm{F}\left(\zeta_{l}\right)$.

5. If $w \mid l$ is a prime of $\mathrm{F}$ then $\left.r\right|_{\mathrm{Gal}\left(\overline{\mathrm{F}}_{w} / \mathrm{F}_{w}\right)}$ is crystalline and for $\tau: \mathrm{F}_{w} \hookrightarrow \overline{\mathbf{Q}}_{l}$ we have

$$
\operatorname{dim}_{\overline{\mathbf{Q}}} \operatorname{gr}^{j}\left(r_{i} \otimes_{\tau, \mathrm{F}_{w}} \mathrm{~B}_{\mathrm{DR}}\right)=1
$$


for $j=0, \ldots, n_{i}-1$ and $=0$ otherwise. Moreover there is a point $t_{w} \in \mathscr{O}_{\mathrm{F}_{w}^{\mathrm{nr}}}$ with $w\left(t_{w}^{n+1}-1\right)=0$ such that

$$
\left.\bar{r}\right|_{\mathrm{I}_{w}} \cong \mathrm{V}_{n}[l]_{t_{w}} .
$$

6. $\left.r\right|_{\mathrm{Gal}\left(\overline{\mathrm{F}}_{v q} / \mathrm{F}_{v q}\right)} ^{\mathrm{ss}}$ is unramified and $\left.r\right|_{\mathrm{Gal}\left(\overline{\mathrm{F}}_{v q} / \mathrm{F}_{v q}\right)} ^{\mathrm{ss}}\left(\operatorname{Frob}_{v_{q}}\right)$ has eigenvalues of the form $\alpha$, $\alpha \# k\left(v_{q}\right), \ldots, \alpha\left(\# k\left(v_{q}\right)\right)^{n-1}$.

Then there is a totally real Galois extension $\mathrm{F}^{\prime \prime} / \mathrm{F}$ and a place $w_{q}$ of $\mathrm{F}^{\prime \prime}$ above $v_{q}$ such that each $\left.r\right|_{\mathrm{Gal}\left(\overline{\mathrm{F}} / \mathrm{F}^{\prime \prime}\right)}$ is automorphic of weight 0 and type $\left\{\operatorname{Sp}_{n}(1)\right\}_{\left\{w_{q}\right\}}$.

(See Theorem 3.3 of [HSBT].)

Theorem 6.2. - Let $\mathrm{F}$ and $\mathrm{L}$ be totally real fields. Let $\mathrm{A} / \mathrm{F}$ be an abelian variety of dimension [L: $\mathbf{Q}$ ] and suppose that $i: \mathrm{L} \hookrightarrow \operatorname{End}^{0}(\mathrm{~A} / \mathrm{F})$. Let $\mathscr{N}$ be a finite set of even positive integers. Fix an embedding $\mathrm{L} \hookrightarrow \mathbf{R}$. Suppose that A has multiplicative reduction at some prime $v_{q}$ of $\mathrm{F}$.

There is a Galois totally real field $\mathrm{F}^{\prime} / \mathrm{F}$ such that for any $n \in \mathscr{N}$ and any intermediate field $\mathrm{F}^{\prime} \supset \mathrm{F}^{\prime \prime} \supset \mathrm{F}$ with $\mathrm{F}^{\prime} / \mathrm{F}^{\prime \prime}$ soluble, Symm ${ }^{n-1} \mathrm{~A}$ is automorphic over $\mathrm{F}^{\prime \prime}$.

(See Theorem 4.1 of [HSBT].)

Theorem 6.3. - Let $\mathrm{F}$ and $\mathrm{L}$ be totally real fields. Let $\mathrm{A} / \mathrm{F}$ be an abelian variety of dimension [L: Q] and suppose that $i: \mathrm{L} \hookrightarrow \operatorname{End}^{0}(\mathrm{~A} / \mathrm{F})$. Fix an embedding $\mathrm{L} \hookrightarrow \mathbf{R}$. Suppose that $\mathrm{A}$ has multiplicative reduction at some prime $v_{q}$ of $\mathrm{F}$.

Then for all $m \in \mathbf{Z}_{\geq 1}$ the function $\mathrm{L}\left(\operatorname{Symm}^{m}(\mathrm{~A}, i), s\right)$ has meromorphic continuation to the whole complex plane, satisfies the expected functional equation and is holomorphic and non-zero in $\operatorname{Re} s \geq 1+m / 2$.

(See Theorem 4.2 of [HSBT].)

Theorem 6.4. - Let $\mathrm{F}$ be a totally real field. Let $\mathrm{E} / \mathrm{F}$ be an elliptic curve with multiplicative reduction at some prime $v_{q}$ of $\mathrm{F}$. The numbers

$$
(1+\mathbf{N} v-\# \mathrm{E}(k(v))) / 2 \sqrt{\mathbf{N} v}
$$

as $v$ ranged over the primes of $\mathrm{F}$ are equidistributed in $[-1,1]$ with respect to the measure $(2 / \pi) \sqrt{1-t^{2}} d t$.

\section{(See Theorem 4.3 of [HSBT].)}

Finally let us consider the $\mathrm{L}$-functions of the motives $\mathrm{V}_{t}$ for $t \in \mathbf{Q}$. More precisely for each pair of rational primes $l$ and $p$ there is a Weil-Deligne representation $\mathrm{WD}\left(\left.\mathrm{V}_{l, t}\right|_{\mathrm{Gal}\left(\overline{\mathbf{Q}}_{p} / \mathbf{Q}_{p}\right)}\right)$ of $\mathrm{W}_{\mathbf{Q}_{p}}$ associated to the $\operatorname{Gal}\left(\overline{\mathbf{Q}}_{p} / \mathbf{Q}_{p}\right)$-module $\mathrm{V}_{l, t}$ (see 
for instance [TY]). Moreover for all but finitely many $p$ there is a Weil-Deligne representation $\mathrm{WD}_{p}\left(\mathrm{~V}_{t}\right)$ of $\mathrm{W}_{\mathbf{Q}_{p}}$ over $\overline{\mathbf{Q}}$ such that for each prime $l \neq p$ and each embedding $\overline{\mathbf{Q}} \hookrightarrow \overline{\mathbf{Q}}_{l}$ the Weil-Deligne representation $\mathrm{WD}_{p}\left(\mathrm{~V}_{t}\right)$ is equivalent to the Frobenius semi-simplification $\mathrm{WD}\left(\left.\mathrm{V}_{l, t}\right|_{\mathrm{Gal}\left(\overline{\mathbf{Q}}_{p} / \mathbf{Q}_{p}\right)}\right)^{\mathrm{F}-\mathrm{ss}}$. We will let $\mathrm{S}\left(\mathrm{V}_{t}\right)$ denote the finite set of primes $p$ for which no such representation $\mathrm{WD}_{p}\left(\mathrm{~V}_{t}\right)$ exists. It is expected that $\mathrm{S}\left(\mathrm{V}_{t}\right)=\emptyset$. If indeed $\mathrm{S}\left(\mathrm{V}_{t}\right)=\emptyset$, then we set $\mathrm{L}\left(\mathrm{V}_{t}, s\right)$ equal to

$$
2^{n / 2}(2 \pi)^{n(n-2) / 8}(2 \pi)^{-n s / 2} \Gamma(s) \Gamma(s-1) \ldots \Gamma(s+1-n / 2) \prod_{p} \mathrm{~L}\left(\mathrm{WD}_{p}\left(\mathrm{~V}_{t}\right), s\right)
$$

and

$$
\epsilon\left(\mathrm{V}_{t}, s\right)=i^{-n / 2} \prod_{p} \epsilon\left(\mathrm{WD}_{p}\left(\mathrm{~V}_{t}\right), \psi_{p}, v_{p}, s\right),
$$

where $v_{p}$ is the additive Haar measure on $\mathbf{Q}_{p}$ defined by $v_{p}\left(\mathbf{Z}_{p}\right)=1$, and $\psi_{p}$ : $\mathbf{Q}_{p} \rightarrow \mathbf{G}$ is the continuous homomorphism defined by

$$
\psi_{p}(x+y)=e^{-2 \pi i x}
$$

for $x \in \mathbf{Z}[1 / p]$ and $y \in \mathbf{Z}_{p}$. The function $\epsilon\left(\mathrm{V}_{t}, s\right)$ is entire. The product defining $\mathrm{L}\left(\mathrm{V}_{t}, s\right)$ converges absolutely uniformly in compact subsets of $\operatorname{Re} s>1+m / 2$ and hence gives a holomorphic function in $\operatorname{Re} s>1+m / 2$.

Theorem 6.5. - Suppose that $t \in \mathbf{Q}-\mathbf{Z}[1 /(n+1)]$. Then $\mathrm{S}\left(\mathrm{V}_{t}\right)=\emptyset$ and the function $\mathrm{L}\left(\mathrm{V}_{t}, s\right)$ has meromorphic continuation to the whole complex plane and satisfies the functional equation

$$
\mathrm{L}\left(\mathrm{V}_{t}, s\right)=\epsilon\left(\mathrm{V}_{t}, s\right) \mathrm{L}\left(\mathrm{V}_{t}, n-s\right) .
$$

(See Theorem 4.4 of [HSBT].)

\section{REFERENCES}

[AC] J. ARthur and L. Clozel, Simple Algebras, Base Change and the Advanced Theory of the Trace Formula, Ann. Math. Stud., vol. 120, Princeton University Press, 1989.

[BM] G. Breuil and A. Mezard, Multiplicités modulaires et représentations de $\mathrm{GL}_{2}\left(\mathbf{Z}_{p}\right)$ et de $\operatorname{Gal}\left(\overline{\mathbf{Q}}_{p} / \mathbf{Q}_{p}\right)$ en $\ell=p$, Duke Math. J., 115 (2002), 205-310.

[CHT] L. Glozel, M. Harris, and R. Taylor, Automorphy for some $\ell$-adic lifts of automorphic mod $\ell$ Galois representations, Publ. Math., Inst. Hautes Étud. Sci., 108 (2008), 1-181.

[E] D. EIsenbud, Commutative Algebra with a View Towards Algebraic Geometry, Springer, 1994.

[EGA] A. Grothendieck, Eléments de géométrie algébrique. IV. Etude locale des schémas et des morphismes de schémas. III., Publ. Math., Inst. Hautes Étud. Sci., 28 (1966).

[HSBT] M. Harris, N. Shepherd-Barron, and R. Taylor, Ihara's lemma and potential automorphy, Ann. Math., to appear. 
[HT] M. Harris and R. Taylor, The Geometry and Cohomology of some Simple Shimura Varieties, Ann. Math. Stud., vol. 151, Princeton University Press, 2001.

[K] M. Kisin, Moduli of finite flat groups schemes and modularity, Ann. Math., to appear.

[M] H. Matsumura, Commutative Ring Theory, Cambridge University Press, 1986.

[SW] G. Skinner and A. Wiles, Base change and a problem of Serre, Duke Math. f., 107 (2001), 15-25.

[TW] R. TAYlor and A. WILES, Ring-theoretic properties of certain Hecke algebras, Ann. Math., 141 (1995), 553-572.

[TY] R. TAYlor and T. Yoshida, Compatibility of local and global Langlands correspondences, f. Amer. Math. Soc., 20 (2007), 467-493.

[W] A. WiLEs, Modular elliptic curves and Fermat's last theorem, Ann. Math., 141 (1995), 443-551.

R. T.

Department of Mathematics,

Harvard University,

Cambridge, MA 02138,

USA

rtaylor@math.harvard.edu

Manuscrit reçu le 27 mai 2006

publié en ligne le 18 novembre 2008. 